msh-mss Mathématiques et sciences humaines

177 | Printemps 2007

Varia

\title{
Les frontières dialectiques
}

Dialectical boundaries

\section{Stéphane Dugowson}

\section{CpenEdition}

Journals

Édition électronique

URL : http://journals.openedition.org/msh/3908

DOI : $10.4000 /$ msh.3908

ISSN : 1950-682

\section{Éditeur}

Centre d'analyse et de mathématique sociales de l'EHESS

\section{Édition imprimée}

Date de publication : 7 mai 2007

Pagination : 87-152

ISSN : 0987-6936

\section{Référence électronique}

Stéphane Dugowson, "Les frontières dialectiques », Mathématiques et sciences humaines [En ligne]

177 | Printemps 2007, mis en ligne le 21 mai 2007, consulté le 23 juillet 2020. URL : http:// journals.openedition.org/msh/3908; DOI : https://doi.org/10.4000/msh.3908 


\title{
LES FRONTIÈRES DIALECTIQUES
}

\author{
Stéphane DUGOWSON ${ }^{1}$
}

\begin{abstract}
RÉSUMÉ - Le but de cet article est de proposer, sous le terme générique de frontières dialectiques, une généralisation de la notion de frontière, notamment mieux adaptée aux " espaces discrets » que la définition topologique classique. Le cas des « espaces nets » est d'abord considéré puis, après un rappel des travaux récents concernant la formalisation des frontières floues, une typologie partielle des différentes définitions qu'on y trouve est proposée en s'appuyant sur les notions $d$ 'espaces dialectiques flous et d'espaces à frontières floues.
\end{abstract}

MOTS CLÉS - Catégories, Flou, Graphe, Prétopologie, Topologie, Treillis

SUMMARY - Dialectical boundaries

The aim of this paper is to propose, under the generic term of dialectical boundaries, a generalization of the concept of boundary, better suited to discrete spaces than the classical topological one. We shall consider first the case of crisp spaces then, after recalling the recent works about the formalization of fuzzy boundaries, we propose a partial typology of the various definitions that they contain, based on the concepts of fuzzy dialectical spaces and of spaces with fuzzy boundaries.

KEYWORDS - Category, Fuzzy, Graph, Lattice, Pretopology, Topology

De nouveaux efforts de conceptualisation des questions et de collecte des données sur les frontières seront une tâche prioritaire au prochain millénaire.

Malcolm Anderson [1997]

\section{INTRODUCTION}

On ne saurait limiter la portée du concept de frontière à un champ particulier du savoir : en toutes disciplines, la connaissance d'un objet implique nécessairement, en quelque façon, celle de ce qui le sépare et le relie à ce qu'il n'est pas, c'est-à-dire celle de ses frontières. Ainsi, toute connaissance est-elle, en un sens, celle de frontières, et ce concept se trouve-t-il d'emblée appelé à traverser les limites disciplinaires.

La notion de frontière d'une partie d'un espace est l'objet d'une définition classique en topologie générale. Cependant, cette définition s'avère insuffisante dans le

\footnotetext{
${ }^{1}$ Institut supérieur de mécanique de Paris, 3 rue Fernand Hainaut, 93407 Saint Ouen Cedex, dugowson@ext.jussieu.fr
} 
cadre d'espaces « discrets » (ou digitaux), tels que les graphes, ou lorsque l'on fait intervenir le flou, comme c'est souvent le cas en analyse automatique des images. Le principal objectif de cet article est de proposer, sous le terme générique de frontières dialectiques, une généralisation de la notion de frontière incorporant notamment le cas de ces espaces « discrets » et conduisant dans le cas des frontières floues à une typologie partielle des différentes définitions proposées en la matière depuis la fin des années 1970.

La première partie, très courte, est consacrée à la définition topologique usuelle des frontières. Elle s'ouvre sur un rappel historique de la définition posée en 1906 par F. Riesz dans le cadre de sa théorie topologique, se poursuit par une caractérisation directe des frontières topologiques et caractérise pour conclure les applications continues en termes de frontières.

L'objet principal de la deuxième partie est d'introduire, dans le seul cas net, l'idée des frontières non symétriques en relation avec le concept d'espace dialectique. Elle contient cinq sous-sections : la première présente le jeu de go, jeu territorial à l'origine d'une grande partie des questions abordées dans cet article; la seconde est consacrée à la notion d'espace connectif, et l'on y trouvera notamment l'énoncé (p. 96) d'une conjecture relative à la représentabilité par entrelacs des espaces connectifs finis et dont le nœud borroméen considéré par Jacques Lacan constitue l'un des exemples le plus fameux, ainsi que l'expression d'un lien surprenant avec les fractals; la troisième sous-section cherche à préciser dans quelle mesure la donnée d'une structure connective entraîne ou, à tout le moins, s'avère compatible avec celle d'un opérateur frontière; la quatrième sous-section rappelle une généralisation simple des espaces topologiques connue sous le nom d'espaces prétopologiques et propose d'y associer la notion de frontière prétopologique; enfin, reprenant l'exemple des frontières au jeu de go, la cinquième sous-section de cette partie considère le cas de frontières non symétriques, montre que cette absence de symétrie se traduit par la nécessité de faire appel à des structures définies par des couples de prétopologies, structures que nous qualifions de dialectiques, examine les relations entre ces structures dialectiques et les structures connectives considérées précédemment et propose enfin d'illustrer la notion de frontière dialectique par quelques exemples, entre autres issus des sciences humaines.

Dans l'introduction de la troisième partie (p.112), nous montrons en quoi la considération des frontières nettes doit nécessairement nous conduire à celle des frontières floues, qui constituent donc l'objet essentiel de cette troisième partie, contenant six sous-sections : la première sous-section de cette partie rappelle les notions d'ensembles et sous-ensembles flous et fixe les notations; la seconde aborde le problème de la négation et de la complémentation dans le cadre des ensembles flous; la troisième présente rapidement la notion de topologie floue; après quelques mots sur la 《préhistoire »des frontières floues, on se propose dans la quatrième sous-section (à partir de la p. 117) de passer en revue les différentes définitions des frontières floues qui ont été proposées respectivement par Liu et $\mathrm{Pu}$ [1976], Warren [1977], Pu [1982], Dubois et Prade [1981], Adnadjevič [1986], Liu [1987], Wu et Zheng [1987], Ying [1987], Cuchillo-Ibanez et Tarrés [1997]; dans la cinquième sous-section nous considérons la notion de frontière prétopologique floue, et enfin les espaces 
dialectiques flous et les espaces à frontières floues, incluant la plupart des définitions antérieures et permettant d'en proposer une classification, sont présentés dans la sixième et dernière sous-section de cette partie.

La conclusion de l'article (p.132) est suivie de plusieurs appendices, auquel le corps de l'article renvoie à diverses reprises. Le premier d'entre eux précise le type de séparation qui, parmi les espaces topologiques, caractérise les espaces de Riesz. Le second donne un exemple de topologie sur $\mathbf{R}$, distincte de la topologie usuelle mais admettant les mêmes connexes. Les troisième et quatrième appendices représentent une part conséquente de l'article; ils contiennent l'ensemble des notions catégorielles constituant le cadre naturel de nos recherches sur les frontières mais que, par souci de faciliter la réception de cet article, nous auront été conduit à rassembler en dehors du corps principal du texte. Plus précisément, le troisième appendice présente la notion de catégorie à treillis de structures (p.134), tandis que le quatrième appendice décrit rapidement une liste d'exemples de telles catégories à treillis de structures : d'abord les prédicats, les graphes, les relations d'équivalence, les espaces connectifs, les espaces prétopologiques, les espaces topologiques, les espaces dialectiques puis, après avoir précisé quelques foncteurs entre les catégories précédentes (p. 142), les ensembles flous, les espaces prétopologiques flous, les espaces topologiques flous, les espaces dialectiques flous et les espaces à frontières floues. Enfin, dans le cinquième appendice on récapitule brièvement les correspondances entre les dénominations employées par divers auteurs depuis les années 1990 en matières d'espaces prétopologiques, bitopologiques, suprabitopologiques, etc.

Un index des notations utilisées est proposé à la fin de l'article, suivi d'une bibliographie.

\section{FRONTIËRES TOPOLOGIQUES}

\subsection{F. Riesz ET LA DÉFinition topologique DES Frontières}

Lors de sa conférence du 22 janvier 1906 à l'Académie hongroise des sciences intitulée Die Genesis des Raumbegriffes ${ }^{2}$ [Riesz, 1907, 1909], le mathématicien hongrois Frigyes ${ }^{3}$ Riesz posait les bases d'une formalisation axiomatique de la topologie générale : indépendante de toute idée de métrique et ne faisant aucune hypothèse quant à la nature des ensembles considérés, cette axiomatique repose sur la notion de points d'accumulation (Verdichtungsstelle) d'une partie; les points de l'espace qui ne sont pas des points d'accumulation d'une partie sont dit isolés vis-à-vis de cette partie. À partir de ces notions, sont alors définis les points intérieurs d'une partie - ce sont ceux des points de cette partie qui ne sont pas des points d'accumulation de son complémentaire - puis les sous-ensembles ouverts. Les éléments d'une partie qui ne lui sont pas intérieurs sont appelés périphériques (Randelemente). La frontière (Grenze) d'une partie est alors constituée de ses éléments périphériques ainsi que de ceux qui sont périphériques à la partie complémentaire.

\footnotetext{
${ }^{2}$ La genèse de la notion d'étendue.

${ }^{3}$ Friedrich en allemand, et Frédéric, en français.
} 
Les propriétés exigées par F. Riesz pour les points d'accumulation ${ }^{4}$ conduisent à une définition équivalente à celle d'espaces topologiques soumis à une contrainte de séparation strictement intermédiaire entre celle des espaces dits accessibles ou de Fréchet, encore appelés espaces $\mathrm{T}_{1}$, c'est-à-dire les espaces topologiques dans lesquels les singletons sont fermés, et celle des espaces séparés ou de Hausdorff, encore appelés espaces $\mathrm{T}_{2}$, c'est-à-dire les espaces topologiques dans lesquels tout couple de points distincts peut être plongé dans un couple d'ouverts disjoints ${ }^{5}$.

Ces espaces de Riesz ${ }^{6}$ sont ainsi caractérisés par une propriété de séparation intermédiaire $\left(\mathrm{T}_{1 \frac{1}{2}}\right.$ ?). Dans ce contexte, la définition des frontières données par Riesz peut s'écrire

$$
\operatorname{Fr}(A)=\left(A \backslash \alpha^{c}(A)\right) \cup\left(\neg A \backslash \alpha^{c}(\neg A)\right)
$$

où nous désignons par $\alpha$ l'opérateur d'adhérence définie en ajoutant ses points d'accumulation à toute partie de l'espace de travail $X, \neg$ l'opérateur de complémentation dans $X$, et $\alpha^{c}$ l'opérateur intérieur associé à l'adhérence $\alpha$, ces deux derniers étant respectivement définis pour toute partie $A$ par

$$
\neg A=X \backslash A=\{x \in X, x \notin A\}
$$

et

$$
\alpha^{c}(A)=\neg \alpha(\neg A) .
$$

On vérifie facilement que la formule (1) équivaut à la formule classique

$$
\operatorname{Fr}(A)=\alpha(A) \cap \alpha(\neg A)
$$

dans laquelle $\alpha$ désigne toujours l'opérateur d'adhérence, associé ici à la topologie de l'espace dans lequel on travaille.

Le travail de Frigyes Riesz restera longtemps méconnu, mais les définitions topologiques ultérieures des frontières seront en tout état de cause équivalentes à la sienne et ne représenteront donc pas d'avancée théorique significative à cet égard ${ }^{7}$. Dans son très bel ouvrage sur les espaces abstraits, Maurice Fréchet [1928, p. 175] écrit par exemple :

La frontière d'un ensemble E est composée de ${ }^{8}$ points de l'espace considéré, qui ne sont ni intérieurs, ni extérieurs à E. Ou encore, un point a appartient à la frontière

\footnotetext{
${ }^{4}$ À savoir : vis-à-vis d'une partie finie, tous les points de l'espace sont isolés ; un point d'accumulation pour une partie l'est encore pour une partie contenant la première; un point d'accumulation pour l'union de deux parties l'est encore pour au moins l'une des deux; une partie admettant un point d'accumulation, il existe une partie de cette partie admettant encore ce point comme point d'accumulation mais vis-à-vis de laquelle tout autre point donné par avance sera isolé.

${ }^{5}$ Sur les différentes notions de séparation pour les espaces topologiques, cf. par exemple le fascicule de Guenard et Lelièvre [1985].

${ }^{6}$ Cf. en appendice, p. 133, Les espaces de Riesz.

${ }^{7}$ A noter toutefois, un peu en marge de la question de la définition des opérateurs frontière, la définition posée en 1920, dans sa thèse, par Kuratowski [1920, 1955, 1961, p. 125] des ensembles frontières (boundary sets), à savoir les parties d'un espace topologique dont le complémentaire est dense dans cet espace.

${ }^{8}$ On aurait pu s'attendre à ce que Fréchet écrive ici des, plutôt que de.
} 
de $E$, si tout voisinage de a contient au moins un point de $E$ et au moins un point n'appartenant pas à $E$.

Ainsi, au début du $\mathrm{XX}^{\mathrm{e}}$ siècle, la formalisation mathématique des frontières en inscrit le concept dans le cadre de la topologie générale, la donnée d'un opérateur frontière résultant de celle d'une structure d'espace topologique considérée comme première (et classiquement caractérisée par les ouverts).

\subsection{DÉFINITION DIRECTE}

Plutôt que de dériver la définition des frontières d'un autre concept topologique, il est possible de caractériser une topologie sur un ensemble non par ses ouverts (selon le procédé habituel) ou par ses points d'accumulation (selon le procédé de Riesz) ou par les adhérences (selon le procédé de Kazimierz Kuratowski [1920] que viendra généraliser la prétopologie ${ }^{9}$ ), mais par son opérateur frontière. La proposition suivante précise les propriétés que doit vérifier une application agissant sur les parties d'un ensemble pour constituer un tel opérateur.

Proposition 1. Soit $X$ un ensemble et $\varphi: \mathcal{P}(X) \rightarrow \mathcal{P}(X)$, une application de l'ensemble des parties de $X$ dans lui-même. On a l'équivalence

$\varphi$ est la frontière associée à une structure topologique sur $X$

$$
\begin{gathered}
\Longleftrightarrow \\
\begin{cases}\text { 1. } & \varphi(\emptyset)=\emptyset \\
\text { 2. } & \forall(A, B) \in \mathcal{P}(X)^{2}, \varphi(A) \subset A \cup B \cup \varphi(A \cup B) \\
\text { 3. } & \forall(A, B) \in \mathcal{P}(X)^{2}, \varphi(A \cup B) \subset \varphi(A) \cup \varphi(B) \\
\text { 4. } & \varphi^{2} \subset \varphi \\
5 . & \varphi \neg=\varphi .\end{cases}
\end{gathered}
$$

DÉMOnTRATIOn. Soit $\varphi$ la frontière associée à une structure topologique sur $X$ dont l'adhérence sera notée $\alpha$. Puisque $\varphi=\alpha \cap \alpha \neg$, la première propriété est assurée, de même que la cinquième (condition de symétrie). Et puisque $\alpha=I d \cup \varphi$, la seconde est la simple traduction en termes de frontières de la propriété $\alpha(A) \subset \alpha(A \cup B)$; la troisième découle de la propriété $\alpha(A \cup B) \subset \alpha(A) \cup \alpha(B)$, qui permet d'écrire

$$
\begin{aligned}
\varphi(A \cup B) & =\alpha(A \cup B) \cap \alpha(\neg A \cap \neg B) \\
& \subset(\alpha(A) \cap \alpha(\neg A \cap \neg B)) \cup(\alpha(B) \cap \alpha(\neg A \cap \neg B)) \\
& \subset(\alpha(A) \cap \alpha(\neg A)) \cup(\alpha(B) \cap \alpha(\neg B)) \\
& =\varphi(A) \cup \varphi(B)
\end{aligned}
$$

tandis que la quatrième résulte, entre autres, de $\alpha^{2}=\alpha$, puisque

$$
\varphi^{2} \subset \alpha \varphi \subset \alpha^{2} \cap \alpha^{2} \neg=\varphi .
$$

\footnotetext{
${ }^{9} \mathrm{Cf}$. la section 2.4 .
} 
Réciproquement, soit $\varphi$ vérifiant les cinq propriétés ci-dessus. Posons $\alpha=I d \cup \varphi$. On a évidemment $I d \subset \alpha$ et, de la première des cinq propriétés considérées, on déduit immédiatement $\alpha(\emptyset)=\emptyset$. La seconde propriété exigée pour $\varphi$ conduit à

$$
\forall(A, B) \in \mathcal{P}(X)^{2}, \alpha(A)=A \cup \varphi(A) \subset A \cup B \cup \varphi(A \cup B)=\alpha(A \cup B),
$$

et la troisième à

$$
\forall(A, B) \in \mathcal{P}(X)^{2}, \alpha(A \cup B) \subset \alpha(A) \cup \alpha(B)
$$

de sorte qu'il y a en fait égalité. On a encore

$$
\begin{aligned}
\alpha^{2} & =(I d \cup \varphi) \alpha=\alpha \cup \varphi \alpha \\
& =\alpha \cup \varphi(I d \cup \varphi) \subset \alpha \cup \varphi \cup \varphi^{2}
\end{aligned}
$$

d'où, puisque $\varphi^{2} \subset \varphi \subset \alpha$

$$
\alpha^{2} \subset \alpha
$$

l'inclusion réciproque découlant de $I d \subset \alpha$. Ainsi, $\alpha$ vérifie les propriétés qui caractérisent une adhérence topologique (cf. section 2.4.)

$$
\left\{\begin{array}{l}
\alpha(\emptyset)=\emptyset \\
\forall(A, B) \in \mathcal{P}(X)^{2}, \alpha(A \cup B)=\alpha(A) \cup \alpha(B) \\
\alpha^{2}=\alpha
\end{array}\right.
$$

Enfin, d'après la cinquième propriété, on a

$$
\alpha \cap \alpha \neg=(I d \cup \varphi) \cap(I d \cup \varphi) \neg=(I d \cup \varphi) \cap(\neg \cup \varphi)=\emptyset \cup \varphi=\varphi
$$

ce qui prouve que $\varphi$ est bien la frontière associée à une structure topologique, à savoir celle caractérisée par $\alpha$.

\subsection{APPLICATIONS CONTINUES}

La donnée d'une application de $\mathcal{P}(X)$ dans lui-même vérifiant les conditions de la proposition 1 peut ainsi être considérée comme une façon de définir, par les frontières, une structure topologique sur $X$. Bien entendu, il est dès lors possible de caractériser les applications continues en termes de frontières.

Proposition 2. Soit $(X, \varphi)$ et $(Y, \psi)$ deux espaces topologiques définis par frontières. Une application $f: X \rightarrow Y$ est continue si et seulement si elle vérifie

$$
f \varphi \subset f \cup \psi f
$$

DÉMONSTRATION. La propriété énoncée est équivalente à $f \cup f \varphi \subset f \cup \psi f$, ou encore à $f(I d \cup \varphi) \subset(I d \cup \psi) f$. Ce qui est la caractérisation en termes d'adhérence des applications continues (cf. la section 2.4.). 


\section{DU GO AUX FRONTIÈRES DIALECTIQUES NETTES}

Beaucoup de notions topologiques semblent devoir exister mathématiquement en dehors du seul cadre de la topologie proprement dite. On pense par exemple à la notion de connexité dans les graphes, dont l'intuition rejoint naturellement celle de la connexité topologique mais qu'il n'est en général pas possible de décrire en termes d'ouverts d'une topologie définie sur l'ensemble des sommets, de sorte qu'elle fait l'objet d'une définition propre aux graphes (on parle parfois de topologie digitale pour désigner l'étude de concepts de style topologique agissant dans des graphes). De même, le très mathématique ${ }^{10}$ jeu de go ${ }^{11}$ fait appel à des frontières qui ne découlent pas d'une structure topologique, mais qui n'en sont pas moins appelées à être mathématisées, et cela même en s'en tenant, comme on le fera dans cette section, aux seules frontières nettes que l'on obtient en fin de partie ${ }^{12}$.

\subsection{LE JEU DE GO}

Inventé en Chine, il y a dit-on près de 4000 ans, le go est un jeu de stratégie dont l'objet est la formation de territoires définies par leurs frontières. Le lecteur francophone pourra trouver les règles du jeu par exemple sur le site de la Fédération Française de Go $^{13}$.

Pour ce qui nous concerne ici, disons simplement que le jeu de go se déroule sur un plateau de jeu, appelé goban, qui est un graphe (simple non orienté). Parmi les notions essentielles : la connexité et, bien que les règles n'y fassent pas nécessairement appel de façon formelle, des frontière qu'il est facile de formaliser dans le cadre d'un graphe quelconque, par exemple sous la forme de ce que nous appellerons la frontière japonaise.

Dans cet article, il sera commode d'utiliser pour les graphes (simples, non orientés) la définition particulière suivante.

DÉFINITION 1. Un graphe est un couple $(G, \mathcal{A})$, où $G$ est un ensemble dont les éléments sont appelés les sommets du graphe, et $\mathcal{A}$ un ensemble de parties de $G$ vérifiant

$$
\left\{\begin{array}{l}
\{\{x\}, x \in G\} \subset \mathcal{A} \\
\forall A \in \mathcal{A}, \operatorname{card}(A) \in\{1,2\}
\end{array}\right.
$$

Les éléments de $\mathcal{A}$ qui ne sont pas des singletons seront appelés les arêtes $d u$ graphe.

Un morphisme d'un graphe $(G, \mathcal{A})$ vers un graphe $(H, \mathcal{B})$ est une application $f: G \rightarrow H$ telle que $\forall A \in \mathcal{A}, f(A) \in \mathcal{B}$.

\footnotetext{
${ }^{10}$ On remarquera au passage que les joueurs de go français, s'ils ne sont pas tous mathématiciens ou informaticiens professionnels, ont pour la plupart fait des études supérieures en mathématiques.

${ }^{11}$ Go est le nom japonais du jeu, appelé Weichi en chinois, et Baduk en coréen.

${ }^{12} \mathrm{En}$ un sens, au jeu de go, les frontières sont affectées d'un flou qu'il s'agit précisément de réduire.

${ }^{13}$ http ://ffg.jeudego.org
} 
L'intérêt d'incorporer les singletons dans l'ensemble $\mathcal{A}$ est la simplification qui en découle quant à la définition des morphismes de graphes. Lorsqu'il n'y aura pas d'ambiguïté, le graphe $(G, \mathcal{A})$ sera simplement noté $G$.

Selon que l'on adopte le point de vue des règles japonaises ou chinoises du jeu de go, la frontière d'une partie du goban correspond à l'une des définitions suivantes.

DÉFInITIOn 2. Soit $(G, \mathcal{A})$ un graphe. La frontière japonaise sur $G$ est l'application $\nu: \mathcal{P}(G) \rightarrow \mathcal{P}(G)$ définie par

$$
\forall A \in \mathcal{P}(G), \nu(A)=\{a \in G, a \notin A \text { et } \exists b \in A,\{a, b\} \in \mathcal{A}\} .
$$

La frontière chinoise sur $G$ est l'application $\mu: \mathcal{P}(G) \rightarrow \mathcal{P}(G)$ définie par

$$
\forall A \in \mathcal{P}(G), \mu(A)=\{a \in G, a \in A \text { et } \exists b \in \neg A,\{a, b\} \in \mathcal{A}\} .
$$

Ces définitions conduisent à des frontières qui ne vérifient plus les conditions de la proposition 1 : si $\varphi$ est la frontière japonaise ou la frontière chinoise sur un graphe $(G, \mathcal{A})$, on aura encore

$$
\begin{aligned}
& \varphi(\emptyset)=\emptyset \\
& \varphi(G)=\emptyset \\
& \forall(A, B) \in \mathcal{P}(G)^{2}, \varphi(A) \subset A \cup B \cup \varphi(A \cup B) \\
& \forall(A, B) \in \mathcal{P}(G)^{2}, \varphi(A \cup B) \subset \varphi(A) \cup \varphi(B)
\end{aligned}
$$

mais on n'aura plus, en général, ni $\varphi^{2} \subset \varphi$, ni $\left.\varphi\right\urcorner=\varphi$.

L'ensemble de ces conditions était la conséquence d'une définition topologique des frontières dont la simplicité et l'élégance ont pu donner le sentiment qu'elle constituait une réponse définitive à la question de la formalisation mathématique des frontières. Pourtant, considérées en elles-mêmes, ces conditions n'ont pas pour elles la force de l'évidence, et l'exemple très simple des frontières dans le jeu de go nous oblige effectivement à les remettre en question. Se pose dès lors la question d'une définition plus générale des frontières, vis-à-vis de laquelle les frontières topologiques, c'est-à-dire les frontières associées aux espaces topologiques, et les frontières chinoises ou japonaises dans les graphes seraient des cas particuliers. Par ailleurs, la considération du jeu de go suggère de s'intéresser non plus seulement aux relations des frontières avec la topologie, mais aussi avec la notion de connexité considérée en toute généralité, posant en préalable la question d'une définition unificatrice de cette dernière notion, par delà la scission qui l'affecte entre graphes et topologie.

\subsection{ESPACES CONNECTIFS}

À notre connaissance, Reinhard Börger [1981, 1983] est le premier a avoir considéré la catégorie des espaces connectifs ("connectivity spaces"), à savoir des espaces dont la structure connective ne découle pas en général d'une topologie ou d'une structure de graphe mais est caractérisée axiomatiquement par le fait que l'union d'une famille de connexes d'intersection non vide est encore connexe. Indépendamment de Börger, Georges Matheron et Jean Serra [1988] ont été conduit à poser la même définition, mais sans adopter un point de vue catégoriel. En 2002, ignorant ces différents 
travaux, j'ai eu à mon tour, au cours d'une partie de go, l'idée de considérer cette catégorie d'espaces [Dugowson, 2005, 2007]. La présente section contient essentiellement la définition des espaces connectifs et quelques exemples, une présentation catégorielle de ces espaces étant proposée en appendice, section 5.4.4.

DÉFINITION 3 (Espaces connectifs). Un espace connectif est un ensemble $X$ muni d'un ensemble $\mathcal{K}$ de parties de $X$ tel que $\emptyset \in \mathcal{K}$ et

$$
\forall \mathcal{I} \in \mathcal{P}(\mathcal{K}), \bigcap_{K \in \mathcal{I}} K \neq \emptyset \Longrightarrow \bigcup_{K \in \mathcal{I}} K \in \mathcal{K} .
$$

Un tel ensemble $\mathcal{K}$ est une structure connective sur $X$, ses éléments sont les parties connexes ou parties connectées de l'espace connectif $(X, \mathcal{K})$. Nous dirons d'un espace connectif qu'il est intègre si tout singleton y est connecté. Un morphisme connectif, ou application connective, d'un espace connectif $(X, \mathcal{K})$ vers un autre $(Y, \mathcal{L})$ est une application $f: X \rightarrow Y$ telle que

$$
\forall K \in \mathcal{K}, f(K) \in \mathcal{L}
$$

On remarque que la notion de parties connexes d'un graphe permet de considérer les graphes comme des espaces connectifs particuliers, les morphismes de graphes ${ }^{14}$ étant alors des applications connectives ${ }^{15}$.

On associe par ailleurs à tout espace topologique un espace connectif en prenant comme structure connective précisément l'ensemble des parties connexes de l'espace considéré, les applications continues étant alors connectives, puisqu'on sait qu'elles préservent la connexité ${ }^{16}$.

Nous dirons d'un espace connectif qu'il est topologique s'il existe un espace topologique auquel il se trouve associé de cette façon ${ }^{17}$. On remarque que cette association est en quelque sorte non injective : par exemple, si on munit $\mathbf{R}$ de la topologie engendrée par la topologie usuelle et l'ensemble $\mathbf{Q}$ des rationnels pris également comme ouvert, on obtient une topologie strictement plus fine mais ayant les mêmes connexes (cf. démonstration en appendice, p. 134, section 5.2.).

L'exemple le plus simple d'espace connectif qui ne soit ni un graphe, ni un espace topologique, est l'espace borroméen $(X, \mathcal{K})$, avec

$$
X=\{1,2,3\} \text { et } \mathcal{K}=\{\emptyset,\{1\},\{2\},\{3\},\{1,2,3\}\} .
$$

Cet espace connectif peut être représenté par l'entrelacs borroméen, connu sous le nom de nœud borroméen. Le psychanalyste Jacques Lacan [1973, 1975] a d'abord

\footnotetext{
${ }^{14} \mathrm{Cf}$. la définition 1.

${ }^{15}$ Autrement dit, la catégorie des graphes est (isomorphe à) une sous-catégorie pleine de celle des espaces connectifs.

${ }^{16}$ Autrement dit, cette association définit un foncteur K de la catégorie des espaces topologiques dans celle des espaces connectifs (cf. la section 5.4.8.).

${ }^{17}$ À noter qu'il existe d'autres façons 《légitimes » que celle ici décrite d'associer un espace connectif à tout espace topologique : on peut par exemple considérer les connexes par arcs; la proposition 3 donne un autre exemple de structure connective associée (notamment) à chaque espace topologique, ce que nous appellerons les connexes aux frontières.
} 
utilisé de tels entrelacs pour figurer les relations entre ce qu'il a appelé le Réel, l'Imaginaire et le Symbolique. Afin notamment d'incorporer à ces figures la notion de sinthome, il fera ensuite appel à divers autres nœuds et entrelacs, en particulier ceux qui vérifient une connectivité globale analogue à celle du borroméen mais qui comportent davantage d'élements (entrelacs brunniens). En référence à cet usage, je propose d'appeller lacanien tout espace connectif fini admettant une représentation dans l'espace usuel $\mathbf{R}^{3}$ sous forme d'un entrelacs, autrement dit d'un ensemble de nœuds disjoints tel qu'une partie non vide de l'espace connectif considéré soit connectée si et seulement si aucun nœud du sous-ensemble correspondant ne peut être mis à l'écart des autres par isotopie ambiante. Lors de mon exposé sur les attractions borroméennes au séminaire Mamuphi de l'École normale supérieure [Dugowson, 2007], j'ai proposé la conjecture qui peut s'énoncer ainsi : « tout espace connectif fini est lacanien ».

Les morphismes connectifs à valeurs dans l'espace borroméen font partie des premiers exemples de morphismes connectifs que l'on est amené à considérer. L'un des plus simples de ce genre est l'application définie sur l'espace connectif obtenu en munissant le plan $\mathbf{R}^{2}$ de la structure connective engendrée par les disques fermés usuels et en associant à tout point de ce plan l'un des trois éléments de l'espace borroméen selon que le point considéré appartient à l'un ou l'autre des trois bassins d'attraction pour la méthode de Newton appliquée dans ce plan, identifié au corps des complexes $\mathbf{C}$, à la recherche des racines cubiques de l'unité, une valeur quelconque pouvant être attribuée aux points se trouvant sur la frontière, appelée ensemble de Julia ${ }^{18}$. Ainsi, chose remarquable, l'un des exemples parmi les plus simples de morphismes connectifs présente donc un aspect fractal [Dugowson, 2006(a), 2007].

\subsection{FRONTIÈRES CONNECTIVES}

Est-il possible d'associer à tout espace connectif $(X, \mathcal{K})$ une application de $\mathcal{P}(X)$ dans lui-même qu'il serait légitime de considérer comme constituant la frontière associée à la structure connective $\mathcal{K}$ sur $X$, selon un procédé qui serait calqué sur l'association à tout espace topologique d'une application frontière ${ }^{19}$ ?

Le fait que deux topologies distinctes, conduisant donc à des frontières distinctes, puissent donner les mêmes connexes ${ }^{20}$, montre que la réponse est négative. Considérons l'exemple élémentaire du graphe formé de deux sommets connectés : $X=\{a, b\}$ et $\mathcal{K}=\mathcal{P}(X)$. La frontière japonaise y est caractérisée par

$$
\nu(\{a\})=\{b\} \text { et } \nu(\{b\})=\{a\},
$$

et la frontière chinoise par

$$
\mu(\{a\})=\{a\} \text { et } \mu(\{b\})=\{b\} .
$$

Par ailleurs, les topologies non discrètes sur $X$, à savoir

\footnotetext{
${ }^{18}$ Une représentation graphique de ce morphisme se trouve par exemple sur la page web http ://www.diffusion.ens.fr/index.php ?res=conf\&idconf=1639.

${ }^{19}$ Cf. la section 1.1 .

${ }^{20} \mathrm{Un}$ exemple en est donné section 5.2.
} 


$$
\begin{aligned}
& \mathcal{T}_{1}=\{\emptyset,\{a\}, X\} \\
& \mathcal{T}_{2}=\{\emptyset,\{b\}, X\} \\
& \mathcal{T}_{3}=\{\emptyset, X\}
\end{aligned}
$$

conduisent toutes trois à la structure connective considérée. Les frontières associées à ces topologies sont définies respectivement par

$$
\begin{aligned}
& \varphi_{1}(\{a\})=\{b\} \text { et } \varphi_{1}(\{b\})=\{b\} \\
& \varphi_{2}(\{a\})=\{a\} \text { et } \varphi_{2}(\{b\})=\{a\} \\
& \varphi_{3}(\{a\})=\varphi_{3}(\{b\})=X .
\end{aligned}
$$

Or, parmi les cinq applications $\mu, \nu, \varphi_{1}, \varphi_{2}$ et $\varphi_{3}$, aucune ne se distingue comme susceptible de représenter la frontière adéquate pour l'espace connectif $(X, \mathcal{K})$. Par contre, toutes cinq vérifient la propriété fondamentale qui, dans le cas topologique, exprime le cœur de ce que nous pourrions appeler la dualité entre les frontières et les passages : dans un espace topologique, toute partie connexe qui rencontre une partie et son complémentaire en rencontre la frontière. D'où l'idée de la définition suivante, par laquelle on renonce à définir la frontière associée à une structure connective pour considérer un ensemble de frontières compatibles avec une telle structure.

DÉFInITION 4 (Frontières connectives). Soit $(X, \mathcal{K})$ un espace connectif. Une $\mathcal{K}$ frontière (ou frontière connective sur cet espace), est une application $\varphi$ de $\mathcal{P}(X)$ dans lui-même vérifiant

$$
\left\{\begin{array}{l}
\varphi(\emptyset)=\varphi(X)=\emptyset \\
\forall A \in \mathcal{P}(X), \forall K \in \mathcal{K},(K \cap A \neq \emptyset \text { et } K \cap \neg A \neq \emptyset) \Longrightarrow K \cap \varphi(A) \neq \emptyset .
\end{array}\right.
$$

Exemple. Soit $(X, \mathcal{K})$ le noud borroméen (cf. plus haut, section 2.2.). L'application $\varphi$ de $\mathcal{P}(X)$ dans lui-même définie par

$$
\left\{\begin{array}{l}
\varphi(\emptyset)=\emptyset \\
\forall a \in X, \varphi(\{a\})=\{a\} \\
\forall A \in \mathcal{P}(X), \varphi(\neg A)=\varphi(A)
\end{array}\right.
$$

est une $\mathcal{K}$-frontière sur $X$. On remarquera que cette frontière, non topologique ${ }^{21}$, vérifie notamment $\varphi(\{1,2\}) \not \subset \varphi(\{1\}) \cup \varphi(\{2\})$.

Étant donnée $\mathcal{K}$ une structure connective sur un ensemble $X$, notons $\Phi_{\mathcal{K}}$ l'ensemble des $\mathcal{K}$-frontières sur $X$. Cet ensemble n'est jamais vide car, en tout état de cause, la frontière grossière (ou indiscrète), définie par

$$
\forall A \in \mathcal{P}(X) \backslash\{\emptyset, X\}, \gamma(A)=X
$$

est nécessairement connective $\operatorname{sur}(X, \mathcal{K})$. Du fait que, pour toute application $\psi$ de $\mathcal{P}(X)$ dans lui-même telle que $\psi(\emptyset)=\psi(X)=\emptyset$, on a trivialement l'implication

$$
\left(\exists \varphi \in \Phi_{\mathcal{K}}, \psi \supset \varphi\right) \Longrightarrow \psi \in \Phi_{\mathcal{K}}
$$

\footnotetext{
${ }^{21}$ C'est-à-dire qu'elle n'est pas associée à une structure topologique.
} 
on pourrait songer à ramener le problème de la détermination des $\mathcal{K}$-frontières à celui de l'existence de $\mathcal{K}$-frontières minimales (pour l'inclusion). Cependant, en général, de telles $\mathcal{K}$-frontières minimales n'existent pas. Il faut néanmoins remarquer qu'une telle condition de minimalité peut fort bien être satisfaite sur un ensemble spécifique de parties de $X$. Par exemple, si $\mathcal{K}$ désigne la structure connective usuelle sur $\mathbf{R}$, on vérifie facilement que pour toute partie $A$ de $\mathbf{R}$ pouvant s'écrire comme réunion finie d'intervalles, toute $\mathcal{K}$-frontière $\varphi$ vérifie nécessairement $\varphi(A) \supset \operatorname{Fr}(A)$, où $\operatorname{Fr}$ désigne la frontière associée à la topologie usuelle, de sorte que pour ces parties-là la frontière usuelle est minimale parmi les $\mathcal{K}$-frontières.

Remarque. Une autre façon d'envisager l'association de frontières aux espaces connectifs sera d'exiger d'une telle association qu'elle soit non plus optimale mais tout au moins régulière, en un sens à préciser ${ }^{22}$.

Si le problème de la détermination des frontières compatibles avec une structure connective donnée est difficile, le problème en quelque sorte dual qui consiste, un opérateur étant donné, à déterminer les structures connectives vis-à-vis desquelles cet opérateur constitue une frontière compatible, s'avère beaucoup plus simple, comme le montre la proposition suivante.

Proposition 3. Soit $\varphi$ une application de $\mathcal{P}(X)$ dans lui-même, telle que $\varphi(\emptyset)=$ $\varphi(X)=\emptyset$. L'ensemble $\mathcal{L}_{\varphi}$ des $K \in \mathcal{P}(X)$ tels que

$$
\forall A \in \mathcal{P}(X),(K \cap A \neq \emptyset \text { et } K \cap \neg A \neq \emptyset) \Longrightarrow K \cap \varphi(A) \neq \emptyset
$$

est une structure connective sur $X$, et pour toute structure connective $\mathcal{K}$ sur $X$, on $a$

$$
\varphi \in \Phi_{\mathcal{K}} \Longleftrightarrow \mathcal{K} \subset \mathcal{L}_{\varphi}
$$

DÉmonstration. Soit $\left(K_{i}\right)_{i \in I}$ une famille dans $\mathcal{L}_{\varphi}$, d'intersection non vide, et $A \in \mathcal{P}(X)$ tel que $\left(\bigcup_{i \in I} K_{i}\right) \cap A \neq \emptyset$ et $\left(\bigcup_{i \in I} K_{i}\right) \cap \neg A \neq \emptyset$. Il s'agit de montrer que $\left(\bigcup_{i \in I} K_{i}\right) \cap \varphi(A) \neq \emptyset$. Notons $x$ un élément de $\bigcap_{i \in I} K_{i}$. Dans le cas où $x \in A$, on considère un $i$ tel que $K_{i} \cap \neg A \neq \emptyset$ : puisqu'on a aussi $K_{i} \cap A \supset\{x\} \neq \emptyset$, on en déduit $K_{i} \cap \varphi(A) \neq \emptyset$, d'où $\left(\bigcup_{i \in I} K_{i}\right) \cap \varphi(A) \neq \emptyset$. Dans le cas où $x \notin A$, on fait appel à un $i$ tel que $K_{i} \cap A \neq \emptyset$ et on conclut de la même façon. Quant à l'équivalence, elle résulte trivialement de la définition de $\Phi_{\mathcal{K}}$.

S'agissant de l'espace $X$ lui-même, remarquons que l'on a $X \in \mathcal{L}_{\varphi}$ si et seulement si aucune partie propre de $X$ n'a sa frontière vide, propriété qui caractérise également la connexité de $X$ dans le cas topologique. La proposition suivante précise un peu la situation dans ce dernier cas.

Proposition 4. Soit $\varphi$ la frontière associée à un espace topologique $X$, et $\mathcal{K}_{\varphi}$ l'ensemble des connexes de cet espace topologique. Alors $\mathcal{K}_{\varphi} \subset \mathcal{L}_{\varphi}$. Par contre, l'inclusion réciproque n'est pas vérifiée pour tout espace topologique.

\footnotetext{
${ }^{22} \mathrm{C}$ 'est par exemple ce que réalise le foncteur $\mathrm{S}$ présenté en section 5.4.8.
} 
DÉmonstration. Cette inclusion exprime la propriété classique déjà mentionnée (cf. par exemple [Bourbaki, 1990, p. I.81]), propriété à l'origine de la définition 4. Pour l'absence de réciproque, donnons un contre-exemple. Soit $X$ un ensemble infini. On y considère la topologie cofinie, pour laquelle les fermés sont, outre $X$, les parties finies. Soit alors $K=\{a, b\}$ une partie de $X$ constituée de deux éléments. $K$ n'est pas connexe pour la topologie considérée, puisque $\{a\}$ et $\{b\}$ sont deux fermés disjoints qui couvrent $K$. Néanmoins, $\{a, b\} \in \mathcal{L}_{\varphi}$. En effet, soit $A$ une partie de $X$ telle que $a \in A$ et $b \notin A$. Si $A$ est finie, son intérieur est vide, de sorte que sa frontière contient encore $a$, et $K \cap \varphi(A) \neq \emptyset$. Si $A$ est infini, son adhérence est $X$, donc contient $b$ qui, n'étant pas dans $A$, appartient ainsi à $\varphi(A)$, d'où $K \cap \varphi(A) \neq \emptyset$.

\subsection{ESPACES ET FRONTIÈRES PRÉTOPOLOGIQUES}

Une autre façon d'envisager la généralisation de la notion mathématique de frontière au-delà du cas topologique, comme nous invite à le faire l'exemple des frontières dans un graphe, est de faire appel aux notions d'adhérence et d'intérieur prétopologiques [Brissaud, 1975; Belmandt, 1993], qui généralisent les notions topologiques correspondantes $^{23}$ :

DÉFInition 5. Soit $X$ un ensemble. Une adhérence sur $X$ est une application $\alpha$ de $\mathcal{P}(X)$ dans lui-même telle que

$$
\left\{\begin{array}{l}
\alpha(\emptyset)=\emptyset \\
\forall A \in \mathcal{P}(X), \alpha(A) \supset A
\end{array}\right.
$$

Si de plus a vérifie

$$
\forall(A, B) \in \mathcal{P}(X)^{2}, A \subset B \Longrightarrow \alpha(A) \subset \alpha(B)
$$

elle est dite de type $\mathcal{V}$. Si elle vérifie en outre

$$
\forall(A, B) \in \mathcal{P}(X)^{2}, \alpha(A \cup B)=\alpha(A) \cup \alpha(B)
$$

elle est dite de type $\mathcal{V}_{\mathcal{D}}$. Nous dirons d'une adhérence de type $\mathcal{V}$ qui est idempotente $\left(\alpha^{2}=\alpha\right)$ qu'elle est de type $\mathcal{V}_{\mathcal{I}}$. Nous dirons enfin qu'une adhérence est de type $\mathcal{T}$ si elle est à la fois de type $\mathcal{V}_{\mathcal{I}}$ et $\mathcal{V}_{\mathcal{D}}$.

Un espace prétopologique est un ensemble muni d'une adhérence. Si cette adhérence est d'un type particulier, nous dirons que l'espace prétopologique en question est de ce type. Un morphisme prétopologique, ou application continue, d'un espace prétopologique $(X, \alpha)$ vers un autre $(Y, \beta)$ est une application $f: X \rightarrow Y$ telle que

$$
\forall A \in \mathcal{P}(X), f(\alpha(A)) \subset \beta(f(A)) .
$$

\footnotetext{
${ }^{23}$ Nous renvoyons à l'appendice (section 5.4.5.) pour une présentation catégorielle des espaces prétopologiques, dont découle immédiatement la possibilité de procéder sur ces espaces à toutes les constructions usuelles (produit cartésien, etc.).
} 
Il est à noter que les espaces de certaines des classes considérées ici ont parfois été appelés espaces de clôture. Par ailleurs, on vérifie facilement qu'un espace topologique est essentiellement la même chose qu'un espace prétopologique de type $\mathcal{T}$, l'adhérence d'un tel espace vérifiant précisément les propriétés qui caractérisent les adhérences topologiques, c'est-à-dire celles qui se trouvent associées à une topologie de la façon habituelle. C'est là le point de vue adopté par Kuratowski [1920, 1955, 1961, 1966] dans sa thèse soutenue à Varsovie le 12 mai 1920 «pour obtenir le grade de docteur en philosophie », un espace topologique y étant défini par la donnée d'une fermeture, nom que prend l'adhérence dans ce cas. Soulignons que la caractérisation de la continuité en termes d'adhérence étant « directe », elle s'avère; dans le cas topologique, plus intuitive que la caractérisation classique en termes d'ouverts.

Un espace prétopologique peut aussi être défini par la donnée d'un opérateur intérieur :

DÉFInItion 6. Soit $X$ un ensemble. Un (opérateur) intérieur sur $X$ est une application $\iota$ de $\mathcal{P}(X)$ dans lui-même telle que

$$
\left\{\begin{array}{l}
\iota(X)=X \\
\forall A \in \mathcal{P}(X), \iota(A) \subset A
\end{array}\right.
$$

Les formules

$$
\iota=\alpha^{c}=\neg \alpha \neg
$$

et

$$
\alpha=\iota^{c}=\neg \iota \neg
$$

établissent une bijection entre intérieurs et adhérences sur un ensemble, faisant des intérieurs une alternative aux adhérences pour la définition des structures prétopologiques. En particulier, nous dirons qu'un intérieur $\iota$ est d'un certain type si l'adhérence associée, $\iota^{c}$, est du type en question.

Examinons à présent la question de savoir comment définir les connexes d'un espace prétopologique, autrement dit la question du prolongement aux espaces prétopologiques de l'association considérée à la section 2.2. qui, à tout espace topologique, associe l'espace connectif défini par les parties connexes d'un tel espace. Dans ce but, commençons par remarquer qu'un espace prétopologique $(X, \alpha)$ et une partie $A \subset X$ étant donnés, l'espace prétopologique $\left(A, \alpha_{A}\right)$ induit sur $A$ par $(X, \alpha)$ doit être défini ${ }^{24}$ par

$$
\forall B \in \mathcal{P}(A), \alpha_{A}(B)=\alpha(B) \cap A .
$$

Ceci étant, s'il existe une partie propre $B$ de $X$ telle que $B$ et $\neg B$ soient «déconnectées» vis-à-vis de l'adhérence, autrement dit si $\alpha(B)=B$ et $\alpha(\neg B)=\neg B$, l'intuition topologique usuelle voudrait que l'espace prétopologique $(X, \alpha)$ ne soit pas considéré comme connexe. A contrario, si pour toute partie propre $B$ de $X$ on a $\alpha(B) \cap \alpha(\neg B) \neq \emptyset$, nous dirons que $(X, \alpha)$ est manifestement connexe. Notant l'apparition de l'expression $\alpha(B) \cap \alpha(\neg B)$, similaire à la formule (4), nous poserons la définition suivante.

\footnotetext{
${ }^{24}$ Il s'agit en fait d'une exigence qui résulte du point de vue catégoriel : cf. la section 5.3.5.
} 
DÉFINITION 7. On dit d'une application $\varphi: \mathcal{P}(X) \rightarrow \mathcal{P}(X)$ qu'elle est une frontière prétopologique (respectivement : de type $\mathcal{V}, \mathcal{V}_{\mathcal{D}}, \mathcal{V}_{\mathcal{I}}, \mathcal{T}$ ) s'il existe sur $X$ une adhérence prétopologique $\alpha$ (respectivement : de type $\mathcal{V}, \mathcal{V}_{\mathcal{D}}, \mathcal{V}_{\mathcal{I}}, \mathcal{T}$ ) telle que

$$
\varphi=\alpha \cap \alpha \neg .
$$

On dit alors que $\varphi$ est la frontière associée à l'espace $(X, \alpha)$.

Nous noterons $\Phi_{\mathcal{P}}(X)$ (respectivement $\Phi_{\mathcal{V}}(X), \Phi_{\mathcal{D}}(X), \Phi_{\mathcal{I}}(X), \Phi_{\mathcal{T}}(X)$ ) l'ensemble des frontières prétopologiques (respectivement de type $\mathcal{V}, \mathcal{V}_{\mathcal{D}}, \mathcal{V}_{\mathcal{I}}$ et $\mathcal{T}$ ) sur $X$. La proposition suivante caractérise directement les différents types de frontières prétopologiques, complétant la proposition 1 donnée p. 91.

Proposition 5. Soit $X$ un ensemble et $\varphi: \mathcal{P}(X) \rightarrow \mathcal{P}(X)$. Comme dans la proposition 1 , on considère les propriétés suivantes

$$
\begin{aligned}
& \text { 1. } \varphi(\emptyset)=\emptyset \\
& \text { 2. } \forall(A, B) \in \mathcal{P}(X)^{2}, \varphi(A) \subset A \cup B \cup \varphi(A \cup B) \\
& \text { 3. } \forall(A, B) \in \mathcal{P}(X)^{2}, \varphi(A \cup B) \subset \varphi(A) \cup \varphi(B) \\
& \text { 4. } \varphi^{2} \subset \varphi \\
& \text { 5. } \varphi \neg=\varphi
\end{aligned}
$$

Les différents types de frontières prétopologiques peuvent alors être ainsi caractérisés

$$
\begin{aligned}
\varphi \in \Phi_{\mathcal{P}}(X) & \Leftrightarrow \varphi \text { vérifie les propriétés } 1 \text { et } 5 . \\
\varphi \in \Phi_{\mathcal{V}}(X) & \Leftrightarrow \varphi \text { vérifie les propriétés 1, 2 et } 5 . \\
\varphi \in \Phi_{\mathcal{D}}(X) & \Leftrightarrow \varphi \text { vérifie les propriétés 1, 2, } 3 \text { et } 5 . \\
\varphi \in \Phi_{\mathcal{I}}(X) & \Leftrightarrow \varphi \text { vérifie les propriétés 1, 2, 4 et } 5 . \\
\varphi \in \Phi_{\mathcal{T}}(X) & \Leftrightarrow \varphi \text { vérifie les cinq propriétés } 1 \text { à } 5 .
\end{aligned}
$$

DÉMONSTRATION. On procède comme pour la proposition 1. En particulier, grâce à la propriété 5 , la correspondance bijective entre frontières prétopologiques d'un type donné et adhérences du même type est exprimée par les relations $\varphi=\alpha \cap \alpha \neg$ et $\alpha=I d \cup \varphi$.

D'après ce qui précède, les espaces prétopologiques d'un type donné peuvent être définis directement en terme de frontières. Lorsqu'il n'y aura pas d'ambiguïté, nous noterons $(X, \varphi)$ l'espace prétopologique $(X, \alpha)$ où $\alpha=I d \cup \varphi$. Étant donnés deux espaces prétopologiques définis par leurs frontières $(X, \varphi)$ et $(Y, \psi)$, les morphismes prétopologiques $f$ du premier vers le second seront alors caractérisés comme dans la proposition 2 par

$$
f \varphi \subset f \cup \psi f .
$$

Nous pouvons reprendre maintenant la question de la définition des connexes d'un espace prétopologique $(X, \varphi)$, un tel espace étant à présent caractérisé par sa frontière, $\varphi$. Pour étendre aux parties $A$ de $X$ le critère de connexité manifeste 
dégagé plus haut pour $X$ lui-même - à savoir : $X$ est manifestement connexe s'il n'existe pas de partie $B$ de $X$ telle que $\varphi(B)=\emptyset$ - nous devons faire appel à $\varphi_{A}$, la frontière prétopologique induite $\operatorname{par} \varphi \operatorname{sur} A$. La détermination de $\varphi_{A}$ peut se faire selon au moins deux méthodes.

Première méthode. La correspondance bijective entre les adhérences prétopologiques et les frontières prétopologiques sur un ensemble donné est en fait un isomorphisme entre treillis complets. En effet, si $\varphi$ et $\psi$ sont les frontières prétopologiques respectivement associées aux adhérences $\alpha$ et $\beta$, on a les implications

$$
\varphi \subset \psi \Rightarrow(I d \cup \varphi) \subset(I d \cup \psi) \Rightarrow \alpha \subset \beta \Rightarrow\left\{\begin{array}{l}
\alpha \subset \beta \\
\alpha \neg \subset \beta \neg
\end{array} \Rightarrow \alpha \cap \alpha \neg \subset \beta \cap \beta \neg\right.
$$

d'où l'équivalence

$$
\varphi \subset \psi \Longleftrightarrow \alpha \subset \beta
$$

Dès lors, $\varphi_{A}$ doit nécessairement être la frontière sur $A$ associée à l'adhérence $\alpha_{A}$ induite sur $A$ par $\alpha$. On en déduit que, pour tout $B \in \mathcal{P}(A)$, on a

$$
\begin{aligned}
\varphi_{A}(B) & =\alpha_{A}(B) \cap \alpha_{A}(\neg A B) \\
& =\alpha(B) \cap A \cap \alpha(A \cap \neg B) \\
& =(B \cup \varphi(B)) \cap A \cap(\neg B \cup \varphi(A \cap \neg B)) \\
& =A \cap((B \cap \varphi(A \cap \neg B)) \cup(\varphi(B) \cap \neg B) \cup(\varphi(B) \cap \varphi(A \cap \neg B)))
\end{aligned}
$$

d'où finalement

$$
\left.\varphi_{A}(B)=(B \cap \varphi(A \cap \neg B)) \cup(\varphi(B) \cap A \cap \neg B)\right) .
$$

Deuxième méthode. Nous pouvons aussi raisonner directement en terme de frontières : conformément à la notion générale de structure induite ${ }^{25}$, l'application frontière induite sur $A$ par $\varphi$ est la moins fine des applications frontières sur $A$ qui rende continue (au sens prétopologique) l'injection canonique $j: A \hookrightarrow X$. Or, une frontière $\psi$ sur $A$ telle que $j$ soit continue est une application $\mathcal{P}(A) \rightarrow \mathcal{P}(A)$ telle que

$$
\left\{\begin{array}{l}
\psi(\emptyset)=\emptyset \\
\psi \neg A=\psi \\
j \psi \subset j \cup \varphi j
\end{array}\right.
$$

et, la troisième condition pouvant s'écrire $\psi \subset I d \cup \varphi$, on en déduit

$$
\begin{aligned}
\psi & =\psi \cap \psi \neg A \\
& \subset(I d \cup \varphi) \cap\left(\neg_{A} \cup \varphi \neg_{A}\right) \\
& =\left(I d \cap \varphi \neg_{A}\right) \cup\left(\varphi \cap \neg_{A}\right) \\
& =(I d \cap \varphi(A \cap \neg)) \cup(\varphi \cap A \cap \neg) .
\end{aligned}
$$

\footnotetext{
${ }^{25}$ Cf. en appendice, p. 137 la section 5.3.5.
} 
Comme cette dernière expression définit une application vérifiant les conditions voulues, et puisqu'elle est moins fine que celles qui y sont incluses, il s'agit de $\varphi_{A}$. On retrouve ainsi la formule (6). À noter que si la frontière $\varphi$ est d'un certain type $(\mathcal{V}$, $\mathcal{V}_{\mathcal{D}}, \mathcal{V}_{\mathcal{I}}$ ou $\mathcal{T}$ ), la formule (6) définit sur $A$ une frontière du même type ${ }^{26}$, de sorte qu'elle donne encore $\varphi_{A}$ lorsqu'on se limite aux espaces prétopologiques des types en question.

Nous sommes à présent en mesure de définir les connexes d'un espace prétopologique.

DÉFinition 8 (Connexes d'un espace prétopologique). Soit $(X, \varphi)$ un espace prétopologique défini par son application frontière $\varphi$. On dit qu'une partie $C$ de $X$ est manifestement connexe pour $\varphi$ si elle vérifie

$$
\forall A \in \mathcal{P}^{*}(C), \varphi_{C}(A) \neq \emptyset .
$$

On note $\mathcal{C}_{\varphi}$ l'ensemble des parties manifestement connexes de $X$, et $\mathcal{K}_{\varphi}$ la plus petite structure connective ${ }^{27}$ sur $X$ contenant $\mathcal{C}_{\varphi}$.

On appelle $\left(\varphi\right.$-)connexes de l'espace $(X, \varphi)$ les éléments de $\mathcal{K}_{\varphi}$.

PROPOSITION 6. Étant donnés deux espaces prétopologiques définis par frontières, $(X, \varphi)$ et $(Y, \psi)$, toute application continue du premier vers le second est un morphisme connectif de $\left(X, \mathcal{K}_{\varphi}\right)$ dans $\left(Y, \mathcal{K}_{\psi}\right)$.

DÉmonstration. Soit $f:(X, \varphi) \rightarrow(Y, \psi)$ une application continue, i.e. telle que $f \varphi \subset f \cup \psi f$. Montrons d'abord que $f\left(\mathcal{C}_{\varphi}\right) \subset \mathcal{C}_{\psi}$. Soit donc $C$ une partie manifestement connexe de $(X, \varphi)$, autrement dit telle que

$$
\forall A \in \mathcal{P}(X),\left(\begin{array}{l}
A \neq \emptyset \\
A \subset C \\
A \cap \varphi(C \backslash A)=\emptyset \\
\varphi(A) \cap C \backslash A=\emptyset
\end{array}\right) \Rightarrow A=C .
$$

On veut montrer que $f(C) \in \mathcal{C}_{\psi}$, autrement dit que

$$
\forall Z \in \mathcal{P}(Y),\left(\begin{array}{l}
Z \neq \emptyset \\
Z \subset f(C) \\
Z \cap \psi(f(C) \backslash Z)=\emptyset \\
\psi(Z) \cap f(C) \backslash Z=\emptyset
\end{array}\right) \Rightarrow Z=f(C) .
$$

Soit donc $Z$ une partie non vide de $f(C)$ vérifiant les conditions ci-dessus, et posons $A=f^{-1}(Z) \cap C$. Puisque $Z \subset f(C)$, on a $f(A)=Z$ et $f(C \backslash A)=f(C) \backslash Z$, d'où l'on déduit

$$
\begin{aligned}
f(A \cap \varphi(C \backslash A)) & \subset f(A) \cap f(\varphi(C \backslash A)) \\
& \subset f(A) \cap(f(C \backslash A) \cup \psi(f(C \backslash A))) \\
& =Z \cap \psi(f(C) \backslash Z)=\emptyset
\end{aligned}
$$

\footnotetext{
${ }^{26}$ Cela se vérifie aisément sur l'adhérence induite.

${ }^{27}$ L'ensemble des structures connectives sur un ensemble $X$ est un treillis complet (cf. en appendice, p. 140 la section 5.4.4.), de sorte que l'intersection des structures connectives contenant un ensemble donné de parties est la plus petite structure connective contenant cet ensemble; on peut aussi la considérer comme engendrée par l'ensemble en question [Dugowson, 2003, p. 11].
} 
de sorte que $A \cap \varphi(C \backslash A)=\emptyset$. Par ailleurs

$$
\begin{aligned}
f(\varphi(A) \cap C \backslash A) & \subset f(\varphi(A)) \cap f(C \backslash A) \\
& \subset(f(A) \cup \psi f(A)) \cap(f(C) \backslash Z)) \\
& =\psi(Z) \cap f(C) \backslash Z=\emptyset
\end{aligned}
$$

d'où $\varphi(A) \cap C \backslash A=\emptyset$. On en déduit $A=C$, d'où $Z=f(C)$.

Ainsi, $f\left(\mathcal{C}_{\varphi}\right) \subset \mathcal{C}_{\psi} \subset \mathcal{K}_{\psi}$. Donc $\left\{K \in \mathcal{P}(X), f(K) \in \mathcal{K}_{\psi}\right\}$, dont on vérifie facilement qu'il s'agit d'une structure connective [Dugowson, 2003, p.10], contient $\mathcal{C}_{\varphi}$, donc contient la structure connective engendrée par les parties manifestement connexes, de sorte que l'image par $f$ de tout $\varphi$-connexe est $\psi$-connexe.

La définition 8 suggère qu'il peut exister des parties connexes qui ne soient pas manifestement connexes, autrement dit que $\mathcal{C}_{\varphi}$ n'est pas, en général, une structure connective sur $X$. Donnons-en un exemple. Soit $\varphi$ la frontière prétopologique sur $X=\{1,2,3\}$ caractérisée par

$$
\left\{\begin{array}{l}
\varphi(\{1\})=\{3\} \\
\varphi(\{2\})=\{1\} \\
\varphi(\{3\})=\emptyset
\end{array}\right.
$$

On constate que pour cette frontière, les parties manifestement connexes sont, outre le vide et les singletons, $\{1,2\}$ et $\{1,3\}$, mais non pas $X$ lui-même, qui est donc connexe sans être manifestement connexe. En quelque sorte, le fait que $X$ soit globalement scindé en deux parties aux frontières vides, $\{3\}$ et $\{1,2\}$, n'empêche pas localement les connections entre ces parties (3 est adhérent à 1). Autrement dit, nous sommes ici dans un cas où l'union ne fait pas la force : l'adhérence de $\{1,2\}$ ne contient pas celle de $\{1\}$ (comme si la présence de 2 inhibait la capacité d'adhésion de 1). Autrement dit encore, la frontière prétopologique $\varphi$ n'est pas de type $\mathcal{V}$. À l'évidence, les sociétés sont en permanence le siège de phénomènes de ce genre, ce qui suggère l'importance que pourrait revêtir, notamment dans les sciences humaines, la considération de structures prétopologiques qui ne soient pas de type $\mathcal{V}$ [Belmandt, 1993, p. 20]. Remarquons encore que dans le cas de la frontière $\varphi$ définie ci-dessus, l'ensemble $\mathcal{L}_{\varphi}$ (cf. p. 98 la proposition 3 ) ne comporte que le vide et les singletons, de sorte que pour cette frontière particulière nous avons

$$
\mathcal{L}_{\varphi} \varsubsetneqq \mathcal{C}_{\varphi} \varsubsetneqq \mathcal{K}_{\varphi}
$$

LEMME 7. Si $\varphi$ est une frontière prétopologique de type $\mathcal{V}$ sur $X$, on a, pour toute partie $A$ et toute partie $B$ de $X$,

$$
\varphi_{A}(B \cap A) \subset \varphi(B) .
$$


DÉMONSTRATION.

$$
\begin{aligned}
\varphi_{A}(B \cap A) & =(\varphi(B \cap A) \cap \neg B \cap A) \cup(B \cap A \cap \varphi(\neg B \cap A)) \\
& \subset(\varphi(B \cap A) \cap \neg B) \cup(B \cap \varphi(\neg B \cap A)) \\
& \subset((B \cup \varphi(B)) \cap \neg B) \cup(B \cap(\neg B \cup \varphi(B))) \\
& =\varphi(B)
\end{aligned}
$$

Proposition 8. Si $\varphi$ est une frontière prétopologique de type $\mathcal{V}$ sur $X$, alors

$$
\mathcal{C}_{\varphi}=\mathcal{K}_{\varphi} \subset \mathcal{L}_{\varphi}
$$

DÉmonstration. Soit $\varphi$ une frontière prétopologique de type $\mathcal{V}$. Montrons que $\mathcal{C}_{\varphi}$ est une structure connective sur X, cela établira $\mathcal{C}_{\varphi}=\mathcal{K}_{\varphi}$. Soit donc $\left(C_{i}\right)_{i \in I}$ une famille de parties de $X$ appartenant à $\mathcal{C}_{\varphi}$, d'intersection non vide. Posons

$$
C=\bigcup_{i \in I} C_{i}
$$

Il s'agit de montrer que $C$ est encore dans $\mathcal{C}_{\varphi}$. Soit donc $B$ une partie non vide de $C$ telle que

$$
\varphi_{C}(B)=\emptyset \text {. }
$$

Posons, pour tout $i \in I, B_{i}=B \cap C_{i}$. Pour tout $i \in I, \varphi_{C_{i}}$ étant la frontière induite sur $C_{i}$ par $\varphi_{C}$, on a, en vertu de la proposition 7

$$
\varphi_{C_{i}}\left(B_{i}\right)=\varphi_{C_{i}}\left(B \cap C_{i}\right) \subset \varphi_{C}(B)
$$

de sorte que $\varphi_{C_{i}}\left(B_{i}\right)=\emptyset$. Mais $B$ étant non vide, il existe un indice $j \in I$ tel que $B_{j} \neq \emptyset$, d'où $B_{j}=C_{j}$. Alors, pour tout indice $i, B_{i}=B \cap C_{i} \supset C_{j} \cap C_{i} \neq \emptyset$, d'où $B_{i}=C_{i}$, et $B=C$, ce qu'il fallait démontrer.

Montrons maintenant l'inclusion $\mathcal{C}_{\varphi} \subset \mathcal{L}_{\varphi}$. Soit donc $K \in \mathcal{C}_{\varphi}$ et $A \in \mathcal{P}(X)$ tels que

$$
\left\{\begin{array}{l}
K \cap A \neq \emptyset \\
K \cap \neg A \neq \emptyset
\end{array}\right.
$$

Posons $B=K \cap A$. D'après la proposition 7 , on a $\varphi_{K}(B) \subset \varphi(A) \cap K$, mais puisque $B \neq \emptyset$ et $K \backslash B=K \cap \neg A \neq \emptyset$, on a $\varphi_{K}(B) \neq \emptyset$, donc $\varphi(A) \cap K \neq \emptyset$.

Exemple. Soit $X=\{1,2,3\}$. L'application $\varphi$ définie p. 97 pour illustrer la définition 4 est une frontière prétopologique de type $\mathcal{V}_{\mathcal{I}}$. Les parties connexes de cet espace sont celles qui ne sont pas des paires : la structure connective définie par cette prétopologie est donc celle du nœud borroméen.

Remarque. Dans le cas des espaces prétopologiques de type $\mathcal{V}$, la définition 8 est équivalente à celle donnée en terme de fermeture ${ }^{28}$ par Belmandt [1993, p. 136 \& p. 151] pour les espaces et sous-espaces connexes.

\footnotetext{
${ }^{28}$ Les fermés d'un espace prétopologique sont les parties invariantes par adhérence, et la fermeture d'une partie est, s'il existe, le plus petit fermé contenant cette partie. Pour les adhérences de type $\mathcal{V}$ mais qui ne sont pas de type $\mathcal{V}_{\mathcal{I}}$, la fermeture est une adhérence distincte de l'adhérence initiale.
} 


\subsection{FRONTIÈRES NON SYMÉTRIQUES ET ESPACES DIALECTIQUES}

Les frontières chinoise ou japonaise sur un graphe (cf. la définition 2, p. 94) ne vérifient pas la condition de symétrie $\varphi \neg=\varphi$, aussi la notion de frontière prétopologique s'avère-t-elle insuffisante pour en rendre compte. L'objet de la présente section 2.5. est ainsi de tirer les conséquences de la suppression de cette condition de symétrie, en particulier quant à la définition des morphismes préservant les structures, dites dialectiques, définies par de telles frontières non symétriques, ainsi que pour ce qui concerne les relations entre frontières et connexité.

\subsubsection{Définition des espaces, des morphismes et des frontières dialectiques}

La suppression de la condition de symétrie signifie essentiellement le découplage entre l'adhérence et l'intérieur associés à une frontière. En effet, à toute frontière prétopologique $\varphi$ sur $X$, étaient associés une adhérence $\alpha=I d \cup \varphi$ et un intérieur $\iota=I d \backslash \varphi$ liés par $\iota=\neg \alpha \neg=\alpha^{c}$ et tels que $\varphi=\alpha \backslash \iota$, puisque d'une part

$$
I d \backslash \varphi=I d \cap \neg \varphi=\neg(\neg \cup \varphi)=\neg(\neg \cup \varphi \neg)=\neg(I d \cup \varphi) \neg
$$

et d'autre part

$$
\alpha \backslash \iota=\alpha \cap \neg \neg \alpha \neg=\alpha \cap \alpha \neg=\varphi .
$$

Si maintenant $\alpha$ et $\iota$ ne sont plus liés, une application de la forme $\varphi=\alpha \backslash \iota$ se trouve définie par la donnée de deux structures prétopologiques, à savoir, en terme d'adhérences, d'une part $\alpha_{1}=\alpha$ et d'autre part $\alpha_{2}=\iota^{c}$. La présence de ces deux structures se manifeste par l'existence de deux relations d'équivalence « orthogonales »: l'équivalence extérieure et l'équivalence intérieure, l'intersection d'une classe extérieure et d'une classe intérieure se réduisant dans tous les cas à un singleton :

DÉFInITIOn 9. Deux applications $\varphi$ et $\psi$ de $\mathcal{P}(X)$ dans lui-même sont extérieurement équivalentes si

$$
I d \cup \varphi=I d \cup \psi,
$$

elles sont intérieurement équivalentes si

$$
I d \cap \neg \varphi=I d \cap \neg \psi
$$

Une application est dite interne si elle est extérieurement discrète (extérieurement équivalente à la frontière vide) et externe si elle est intérieurement discrète.

Il apparaît ainsi que la considération de frontières non symétriques équivaut à celle de couples de structures prétopologiques, l'une spécifiant la classe d'équivalence extérieure, l'autre la classe d'équivalence intérieure d'une telle frontière. Les définitions suivantes des espaces dialectiques, des morphismes dialectiques et des frontières dialectiques découlent déjà de cette remarque, même si le chemin suivi aura en fait été catégoriel ${ }^{29}$.

\footnotetext{
${ }^{29}$ Cf. en appendice la section 5.4.7.
} 
DÉFINITION 10 (Espaces dialectiques nets). Une structure dialectique (nette) sur un ensemble $X$ est un couple $\left(\alpha_{1}, \alpha_{2}\right)$ de prétopologies sur $X$. Un espace dialectique est un ensemble $X$ muni d'une structure dialectique. Un morphisme dialectique $f$ d'un tel espace dialectique $\left(X, \alpha_{1}, \alpha_{2}\right)$ vers un autre $\left(Y, \beta_{1}, \beta_{2}\right)$ est une application $f: X \rightarrow Y$ telle que

$$
\forall A \in \mathcal{P}(X), \forall i \in\{1,2\}, f\left(\alpha_{i}(A)\right) \subset \beta_{i}(f(A)) .
$$

On définit de même les espaces dialectiques de différents types en fonction des types d'adhérences prétopologiques employées ${ }^{30}$.

La proposition suivante permet alors de définir la notion de frontière dialectique comme synthèse entre deux logiques organisatrices de l'espace, une logique intérieure et une logique extérieure; plus précisément, elle permet de représenter par un opérateur unique le couple des structures prétopologiques qui caractérisent un espace dialectique.

Proposition 9. Étant donné un ensemble $X$, notons $\Phi_{X}$ l'ensemble

$$
\Phi_{X}=\left\{\varphi \in \mathcal{P}(X)^{\mathcal{P}(X)}, \varphi(\emptyset)=\varphi(X)=\emptyset\right\}
$$

et $\mathcal{D}_{X}$ l'ensemble des structures dialectiques sur $X$. Alors l'application $\theta: \mathcal{D}_{X} \rightarrow \Phi_{X}$ définie par

$$
\left.\forall\left(\alpha_{1}, \alpha_{2}\right) \in \mathcal{D}_{X}, \theta\left(\alpha_{1}, \alpha_{2}\right)=\alpha_{1} \cap \alpha_{2}\right\urcorner
$$

est une bijection, de réciproque $\lambda$ définie par

$$
\forall \varphi \in \Phi_{X}, \lambda(\varphi)=(I d \cup \varphi, I d \cup \varphi \neg) .
$$

DÉmonstration. Pour tout couple $\left(\alpha_{1}, \alpha_{2}\right)$ de prétopologies sur $X$, on a

$$
\lambda \theta\left(\alpha_{1}, \alpha_{2}\right)=\left(I d \cup\left(\alpha_{1} \cap \alpha_{2} \neg\right), I d \cup\left(\alpha_{1} \cap \alpha_{2} \neg\right) \neg\right)=\left(\alpha_{1}, \alpha_{2}\right),
$$

et pour tout $\varphi \in \Phi_{X}$, on a

$$
\theta \lambda(\varphi)=(\varphi \cup I d) \cap(\varphi \neg \cup I d) \neg=\varphi
$$

DÉFINITION 11 (Frontières dialectiques). La frontière dialectique associée à un espace dialectique $\left(X, \alpha_{1}, \alpha_{2}\right)$ est l'application $\varphi$ définie par

$$
\varphi=\alpha_{1} \cap \alpha_{2} \neg .
$$

Proposition 10. Pour toute application $\varphi$ de $\mathcal{P}(X)$ dans lui-même telle que $\varphi(\emptyset)=$ $\varphi(X)=\emptyset$, il existe une et une seule structure dialectique sur $X$ dont la frontière associée soit $\varphi$. De plus, cette structure dialectique est de type $\mathcal{V}$ (vis-à-vis des deux prétopologies) si et seulement si $\varphi$ vérifie

$$
\forall(A, B) \in \mathcal{P}(X)^{2}, A \subset B \Rightarrow\left\{\begin{array}{l}
\varphi(A) \subset B \cup \varphi(B) \\
\varphi(B) \subset \neg A \cup \varphi(A)
\end{array}\right.
$$

\footnotetext{
${ }^{30}$ Définition 5, p. 99.
} 
DÉmonstration. L'existence d'une unique structure dialectique à laquelle $\varphi$ se trouve associée est affirmée par la proposition 9 . Une telle structure est de type $\mathcal{V}$ (vis-à-vis des deux prétopologies) si pour tout $(A, B) \in \mathcal{P}(X)^{2}$ tel que $A \subset B$ et pour $i \in\{1,2\}$, on a $\alpha_{i}(A) \subset \alpha_{i}(B)$. Or, $\alpha_{1}(A) \subset \alpha_{1}(B)$ équivaut à $\varphi(A) \subset B \cup \varphi(B)$, tandis que $\alpha_{2}(A) \subset \alpha_{2}(B)$ équivaut à $\varphi(\neg A) \subset B \cup \varphi(\neg B)$ d'où, en échangeant $A$ et $\neg B$, le résultat annoncé.

Les considérations précédentes conduisent à une définition des espaces dialectiques directement en terme de frontières :

DÉFINITION 12 (Espaces dialectiques par les frontières). Un espace dialectique défini par frontière est un couple $(X, \varphi)$ où $X$ est un ensemble et $\varphi$ une application de $\mathcal{P}(X)$ dans lui-même telle que $\varphi(\emptyset)=\varphi(X)=\emptyset$. Un morphisme d'un tel espace $(X, \varphi)$ vers un autre $(Y, \psi)$ est une application $f: X \rightarrow Y$ telle que

$$
f \varphi \subset(f \cup \psi f) \cap(f \neg \cup \psi \neg f \neg) .
$$

Nous laissons au lecteur le soin de vérifier que cette caractérisation des morphismes dialectiques est équivalente à celle de la définition 10. La possibilité de définir un espace dialectique directement par son opérateur frontière justifie que, dans la suite, nous appelions frontière dialectique sur $X$ tout opérateur de $\mathcal{P}(X)$ dans lui-même telle que $\varphi(\emptyset)=\varphi(X)=\emptyset$, et frontière dialectique d'un type donné toute frontière dialectique telle que la structure dialectique correspondante soit du type en question. La proposition 10 caractérise ainsi, en particulier, les frontières dialectiques de type $\mathcal{V}$ sur $X$, et il est aisé, en faisant appel à des propriétés analogues à celles que la proposition 5 (p. 101) avance pour les frontières symétriques, de caractériser les différents types de frontières dialectiques.

DÉFINITION 13 (Frontières adjointes). Soit $\varphi$ une frontière dialectique sur un ensemble $X$. L'adjointe $\tilde{\varphi}$ est la frontière sur $X$ définie par

$$
\tilde{\varphi}=\varphi \neg .
$$

Ainsi, une frontière dialectique est prétopologique si et seulement si elle est autoadjointe, c'est-à-dire symétrique. L'adjointe d'une frontière externe est interne, et mutatis mutandis.

\subsubsection{Espaces dialectiques et connexité}

D'après les propositions 3 et 10 , un espace connectif $\left(X, \mathcal{L}_{\varphi}\right)$ se trouve naturellement associé à tout espace dialectique $(X, \varphi)$, avec pour structure connective $\mathcal{L}_{\varphi}$ l'ensemble de parties de $X$ défini par

$$
\left.\mathcal{L}_{\varphi}=\left\{K \in \mathcal{P}(X), \forall A \in \mathcal{P}(X), \begin{array}{r}
K \cap A \neq \emptyset \\
K \cap \neg A \neq \emptyset
\end{array}\right\} \Longrightarrow K \cap \varphi(A) \neq \emptyset\right\}
$$

Nous dirons que les éléments de $\mathcal{L}_{\varphi}$ sont les connexes aux frontières associés à l'opérateur frontière $\varphi$. 
Par ailleurs, la définition des ensembles $\mathcal{C}_{\varphi}$ et $\mathcal{K}_{\varphi}$ (introduits dans le cas des frontières prétopologiques par la définition 8 p. 103) s'étend au cas des frontières dialectiques à condition de préciser ce qu'est $\varphi_{A}$, l'opérateur frontière induit par $\varphi$ sur $A \subset X$.

Comme pour les considérations qui précèdent la définition 8 , nous pouvons déterminer $\varphi_{A}$ de deux façons, en faisant appel à la restriction des adhérences, ou en raisonnant directement en termes d'espace dialectique. Procédons de cette dernière façon. En accord avec la notion générale de structure induite vue comme structure initiale $^{31}, \varphi_{A}$ est la moins fine des frontières dialectiques sur $A$ qui rende continue - au sens de la formule (8) de la définition 12 - l'injection canonique $j: A \hookrightarrow X$. On en déduit

$$
\varphi_{A} \subset(I d \cup \varphi) \cap\left(\neg_{A} \cup \varphi \neg_{X} \neg_{A}\right),
$$

puis, pour tout $B \subset A$

$$
\varphi_{A}(B)=(\varphi(B) \cap A \cap \neg B) \cup(B \cap \varphi(B \cup \neg A))
$$

La formule (10) généralise la formule (6) obtenue p. 102, à laquelle elle se réduit dans le cas où $\varphi$ est symétrique $(\varphi=\varphi \neg)$. Ceci étant, l'ensemble $\mathcal{C}_{\varphi}$ des parties $m a$ nifestement connexes et l'ensemble $\mathcal{K}_{\varphi}$ des parties connexes d'un espace dialectique $(X, \varphi)$ sont alors définis exactement comme dans la définition 8 pour les espaces prétopologiques

$$
\mathcal{C}_{\varphi}=\left\{C \in \mathcal{P}(X), \forall A \in \mathcal{P}^{*}(C), \varphi_{C}(A) \neq \emptyset\right\}
$$

et $\mathcal{K}_{\varphi}$ est la structure connective engendrée sur $X$ par $\mathcal{C}_{\varphi}$.

PROPOSITION 11. Étant donnée une frontière dialectique $\varphi$ sur un ensemble $X$, on $a$

$$
\left\{\begin{array}{l}
\mathcal{C}_{\varphi}=\mathcal{C}_{\varphi\urcorner} \\
\mathcal{K}_{\varphi}=\mathcal{K}_{\varphi\urcorner} \\
\mathcal{L}_{\varphi}=\mathcal{L}_{\varphi\urcorner}
\end{array}\right.
$$

DÉmonstration. On a, pour tout $C \in \mathcal{P}^{*}(X)$

$$
\begin{aligned}
C \in \mathcal{C}_{\varphi} & \Rightarrow \forall A \in \mathcal{P}^{*}(C),(\varphi(A) \cap C \cap \neg A) \cup(A \cap \varphi(A \cup \neg C)) \neq \emptyset \\
& \Rightarrow \forall A \in \mathcal{P}^{*}(C),(\varphi(\neg A \cap C) \cap A) \cup(\neg A \cap C \cap \varphi(\neg A)) \neq \emptyset \\
& \Rightarrow C \in \mathcal{C}_{\varphi\urcorner}
\end{aligned}
$$

d'où la première affirmation, dont découle immédiatement la seconde. La troisième résulte trivialement de la définition de $\mathcal{L}_{\varphi}$.

Les propositions suivantes 12 à 14 se démontrent respectivement comme les propositions 6 à 8 (p. 103 à 105), en veillant à utiliser la formule (10) au lieu de (6) et, pour la continuité, la formule (8).

${ }^{31}$ Cf. l'appendice 5.3.5., p. 137. 
PROPOSITION 12. Étant donnés deux espaces dialectiques définis par leurs frontières, $(X, \varphi)$ et $(Y, \psi)$, toute application continue du premier vers le second est un morphisme connectif de $\left(X, \mathcal{K}_{\varphi}\right)$ dans $\left(Y, \mathcal{K}_{\psi}\right)$.

LEMme 13. Si $\varphi$ est une frontière dialectique de type $\mathcal{V}$ sur $X$, on a, pour toute partie $A$ et toute partie $B$ de $X$

$$
\varphi_{A}(B \cap A) \subset \varphi(B) .
$$

PRoposition 14. Si $\varphi$ est une frontière dialectique de type $\mathcal{V}$ sur $X$, alors

$$
\mathcal{C}_{\varphi}=\mathcal{K}_{\varphi} \subset \mathcal{L}_{\varphi}
$$

\subsubsection{Exemples d'espaces et de frontières dialectiques}

1. En première approximation, on peut considérer la peau comme (une partie de la) frontière du corps identifié à l'ensemble des cellules vivantes qui le constituent. Etant non symétrique, il s'agit bien d'une frontière dialectique : l'épiderme, constitué de cellules mortes, est en effet moins épais que la réunion du derme et de l'hypoderme, constituée de cellules vivantes et donc intérieure à l'ensemble considéré, de sorte que dans cette approximation l'épiderme correspond à une prétopologie plus fine ${ }^{32}$ que celle correspondant au derme et à l'hypoderme réunis.

2. Dans son article sur les frontières linguistiques, Jacques Leclerc [2006] distingue parmi les frontières linguistiques celles qui sont étanches, poreuses ou asymétriques : les frontières linguistiques étanches correspondent au cas où les groupes linguistiques considérés «ne peuvent pas légalement conserver leur langue en changeant de territoire », comme c'est par exemple le cas en Suisse entre celles qui séparent les régions romande, alémanique et italienne; les frontières linguistiques poreuses sont illustrées par l'exemple canadien du Québec et de l'Ontario, où « un anglophone de l'Ontario a le droit d'envoyer ses enfants dans une école anglaise du Québec comme un francophone a le même droit en Ontario »; enfin, les frontières linguistiques asymétriques, étanches ou poreuses selon les cas, sont illustrées par l'exemple de la Chine, où « on donne la possibilité à la langue majoritaire (mandarin ou chinois officiel), mais non aux langues minoritaires (mongol, tibétain, ouïgour, etc.) de changer de territoire ». On peut modéliser cette dernière situation de la façon suivante : sur l'ensemble $X=\{M, O, T\}$ (pour simplifier, nous nous limitons à trois langues : $M$ pour mandarin, $O$ pour oü̈gour et $T$ pour tibétain), l'adhérence $\alpha$ de type $\mathcal{V}$ correspondant à la description de J.Leclerc sera définie pour toute partie $Y$ de $X$ par $\alpha(Y)=X$ si $M \in Y$ et $\alpha(Y)=Y$ si $M \notin Y$, tandis que l'intérieur $\iota$ de type $\mathcal{V}$ découlant de cette description sera défini pour les parties propres de $X$ par $\iota(Y)=\{M\}$ si $M \in Y$ et $\iota(Y)=\emptyset$ si $M \notin Y$. La frontière $\varphi$ associée à cette structure dialectique est telle que, pour les parties propres de $X$, la frontière d'une partie comportant $M$ sera $\{O, T\}$, tandis qu'une partie ne comportant par $M$ sera sa propre frontière.

\footnotetext{
${ }^{32}$ Sur les notions d'épaisseur ou de finesse des prétopologies ou de structures analogues, cf. la section 5.3.1.
} 
On vérifie facilement qu'une telle frontière n'est pas symétrique : il s'agit bien d'une frontière dialectique.

3. Dans le même ordre d'idées, on peut reconnaître la présence de deux prétopologies distinctes - donc d'un espace dialectique, même si les prétopologies ne semblent pas ici correspondre au couple intérieur/adhérence - dans certaines descriptions par Edward T. Hall des différences culturelles dans le rapport à l'espace, comme dans ce passage, extrait de son ouvrage Le langage silencieux [1959] :

En Amérique latine, la distance d'interaction est beaucoup moins élevée qu'aux EtatsUnis. En fait les gens ne se sentent à l'aise pour parler que lorsqu'ils se rapprochent de la distance qui, aux USA, évoquent le sexe ou l'agressivité. Il s'ensuit que lorsqu'ils se rapprochent, nous reculons et ainsi de suite. Ils pensent que nous sommes distants ou froids, renfermés et inamicaux. De notre coté, nous leur reprochons de nous souffler dans les oreilles, de nous envahir, de nous postillonner dans la figure.

Les Américains qui ont vécu en Amérique Latine sans être conscients de ces attitudes vis-à-vis de l'espace s'adaptent autrement, en se barricadant derrière leur bureau, en utilisant des chaises et des machines à écrire pour sauvegarder entre eux et les Latins une distance qu'ils trouvent confortable. Le résultat est que le Latin risque d'enjamber les obstacles pour réduire la distance et se sentir plus à l'aise.

4. Soit $(G, \mathcal{A})$ un graphe ${ }^{33}$. L'adhérence $\alpha$ sur $G$ qui lui est naturellement associée, à savoir l'application de $\mathcal{P}(G)$ dans lui-même définie par

$$
\forall A \in \mathcal{P}(G), \alpha(A)=\{a \in G, \exists b \in A,\{a, b\} \in \mathcal{A}\}
$$

est une adhérence prétopologique de type $\mathcal{V}_{\mathcal{D}}$. Ce qu'inspiré par le jeu de go nous avons appelé la frontière chinoise sur $G$ vérifie

$$
\mu=I d \cap \alpha \neg
$$

tandis que pour la frontière japonaise on a

$$
\nu=\alpha \cap \neg .
$$

$I d$, l'adhérence discrète, étant de tous types, les frontières chinoise et japonaise sur un graphe sont ainsi des frontières dialectiques de type $\mathcal{V}_{\mathcal{D}}$, adjointes l'une de l'autre, la première interne, la seconde externe.

Si maintenant $\mathcal{K}$ désigne l'ensemble des parties $K$ de $\mathcal{P}(X)$ telles que pour tout couple $(x, y)$ d'éléments de $K$ il existe une suite finie $x_{0}, x_{1}, \ldots, x_{n}$ telle que $x_{0}=x$, $x_{n}=y$ et, pour tout indice $i \in\{0, \ldots, n-1\},\left\{x_{i}, x_{i+1}\right\} \in \mathcal{A}$, on vérifie que $\mathcal{L}_{\nu} \subset \mathcal{K} \subset \mathcal{C}_{\nu}$, de sorte que, d'après les propositions précédentes

$$
\mathcal{C}_{\nu}=\mathcal{K}_{\nu}=\mathcal{L}_{\nu}=\mathcal{K}=\mathcal{C}_{\mu}=\mathcal{K}_{\mu}=\mathcal{L}_{\mu}
$$

5. Un espace bitopologique [Kelly, 1962] est un ensemble muni d'un couple de topologies : c'est donc un espace dialectique de type $\mathcal{T}$. Les espaces bitopologiques ${ }^{34}$

\footnotetext{
${ }^{33} \mathrm{Au}$ sens de la définition 1, p. 93.

${ }^{34}$ Les espaces bitopologiques constituent le domaine de recherche référencé 54E55 dans la Classification Mathématique par matières 2000 (MSC 2000).
} 
surgissent par exemple lors de l'étude des espaces quasi-métriques [Wilson, 1931, Albert 1941 ; Ribeiro, 1942 ; Balanzat, 1951 ; Kelly, 1962], c'est-à-dire des ensembles munis d'une « distance » non symétrique, dite quasi-distance, pour laquelle la distance d'un point $A$ à un point $B$ peut donc différer de celle séparant $B$ de $A$. Exactement comme pour une distance classique (mais en faisant attention à l'ordre des termes) on associe une topologie à toute quasi-distance. Se pose alors notamment la question de savoir à quelles conditions un espace topologique est quasi-métrisable. Kelly poursuit l'étude de ce type de questions en considérant simultanément deux topologies sur un ensemble muni d'une quasi-distance, à savoir la topologie associée à cette quasi-distance et celle associée à la quasi-distance conjuguée, i.e. la distance obtenue en échangeant l'ordre des points.

6. Les espaces bitopologiques ont également été mis à contribution dans l'étude des topologies floues sur un ensemble (cf. la définition 16, p. 117) : Brown [1986] représente ainsi une topologie floue par un espace bitopologique; de même Ertürk [1993], qui associe à toute topologie floue dans un treillis flou ${ }^{35}$ une bitopologie sur l'ensemble des molécules ${ }^{36}$ du treillis en question.

\section{FRONTIÈRES FLOUES}

La faillite de l'exigence que les frontières soient nettes (exigence qu'on a vu faire faillite dans le texte de Frege lui-même) ce serait ainsi la faillite de la métaphysique elle-même. À la différence de Derrida, par exemple, qui demande, apparemment, qu'on maintienne l'exigence freguéenne, Wittgenstein nous assure que c'est inutile.

Geoffrey Bennington [1992]

Les considérations des sections précédentes répondent à l'évidence à l'exigence de netteté des frontières posée par Frege, exigence discutée par Geoffrey Bennington dans son intervention sur « la frontière infranchissable » qu'il a présentée lors du colloque Le Passage des Frontières organisé en 1992 à Cerisy autour du travail de Jacques Derrida. Elles y répondent même doublement : d'une part, s'agissant des frontières du concept, parce qu'il ne saurait en être autrement pour un concept faisant l'objet de définitions mathématiques rigoureuses, d'autre part, s'agissant du type de frontières jusqu'à présent considérées, parce que celles-ci sont relatives à des zones précises et constituent elles-mêmes des régions précises des espaces mathématiques considérés, à savoir des ensembles, vis-à-vis desquels le choix est, pour chaque point, toujours binaire : en faire partie, ou non.

Mais au découpage binaire de l'espace induit par la considération d'une région particulière $Z$ d'un tel espace $X$ (à savoir que pour tout $x \in X$ on a soit $x \in Z$, soit

\footnotetext{
${ }^{35}$ Cf. les définitions 17 et 18 , p. 117.

${ }^{36} \mathrm{Cf}$. la définition 25 , p. 125 .
} 
$x \notin Z)$ se superpose, par la considération des frontières (nettes) définies dans les sections précédentes, un autre découpage, cette fois ternaire puisque s'y distinguent l'intérieur, la frontière et l'extérieur de la région considérée (à savoir, pour une frontière dialectique $\varphi=\alpha \cap \neg \iota$, les trois régions $\iota(Z), \varphi(Z)$ et $\neg \alpha(Z))$. Or, un tel découpage ternaire représente l'un des modes d'accès aux théories mathématiques du flou : on y reconnait par exemple la formalisation du flou par Gentilhomme [1968], qui consiste à caractériser un sous-ensemble flou d'un ensemble $X$ donné par un couple $(I, A)$ de sous-ensembles emboîtés $(I \subset A \subset X), I$ étant appelé «zone sûre », $A$ «zone d'extension maximum » et enfin $A \backslash I$ «zone floue ». D'un point de vue topologique, $I$ représente en quelque sorte l'intérieur et $A$ l'adhérence du sous-ensemble flou ainsi défini, de sorte que l'idée de frontière coïncide ici avec celle de « zone floue ». De façon équivalente, la définition de Gentilhomme revient à attribuer à tout point $x \in X$ une valeur prise dans un ensemble qui en comporte trois, disons $L=\left\{0, \frac{1}{2}, 1\right\}$ en convenant qu'à tout point appartenant à $I$ on attribue la valeur 1, qu'à tout point qui n'est pas dans $A$, on attribue la valeur 0 et qu'enfin aux points-frontière constituant la zone floue on attribue la valeur $\frac{1}{2}$.

La considération des frontières (nettes) conduit ainsi naturellement aux ensembles flous, ceux de Gentilhomme constituant un cas particulier de ceux introduits antérieurement ${ }^{37}$ par Zadeh [1965]. La remarque, qui vaut déjà pour les frontières topologiques, est encore plus évidente pour les frontières dialectiques dont la donnée équivaut à un couple d'opérateurs qui renvoie directement au couple d'ensembles considéré par Gentilhomme.

Autrement dit : l'actualisation d'un choix binaire se fait en tranchant, en séparant, mais l'espace-frontière ainsi créé, l'espace de la séparation, représente alors un troisième terme qui potentiellement relie autant qu'il sépare et par où tendent à s'engouffrer les valeurs intermédiaires qui caractérisent le flou.

C'est toutefois par un chemin un peu différent, catégoriel, que s'est imposée à nous l'idée qu'il était nécessaire, dès lors que l'on s'occupait de frontières, de s'intéresser également au flou. Il s'avère en effet que le choix d'un couple de (pré)topologies - en quelque sorte de deux logiques de l'espace - revient essentiellement à effectuer le produit cartésien de treillis complets ${ }^{38}$, opération qui reçoit d'un point de vue catégoriel une interprétation susceptible de concerner tous les types d'espaces considérés dans les sections précédentes, précisément du fait que les structures correspondantes s'organisent, pour chaque ensemble sous-jacent sur lequel elles sont considérées, en treillis complets. Or, l'exemple le plus simple de structures choisies de la sorte dans des treillis complets est précisément celui du flou ${ }^{39}$ défini par Zadeh [1965]. Ainsi le thème des frontières floues s'est-il imposé de lui-même.

Nous pourrions multiplier les exemples qui montrent que, dans de nombreux discours, scientifiques ou non, les frontières sont appelées à évoluer entre les pôles extrêmes de la netteté la plus parfaite et du flou le plus vague. Que le flou des frontières soit constaté (souvent avec étonnement), souhaité (pour des raisons pra-

\footnotetext{
${ }^{37}$ Gentilhomme, dans son article de 1968, ne cite pas l'article de Zadeh [1965] dont à l'évidence il ignore l'existence.

${ }^{38} \mathrm{Cf}$. en appendice la section 5.3.9.

${ }^{39} \mathrm{Cf}$. en appendice la section 5.4.9.
} 
tiques, esthétiques ou, lorsqu'il s'agit d'une option idéologique générale visant la dissolution de toutes frontières, par nihilisme) ou qu'on le regrette, le fait est que, pour qui cherche à mieux cerner le concept de frontière, le flou est rigoureusement incontournable.

\subsection{ENSEMBLES ET SOUS-ENSEMBLES FLOUS : RAPPELS ET NOTATIONS}

La notion d'ensemble flou a ainsi été introduite par Zadeh [1965] : un ensemble $X$ étant donné,

$A$ fuzzy set $A$ in $X$ is characterized by a membership (characteristic) function $f_{A}(x)$ which associates with each point in $X$ a real number in the interval $\mathbf{I}=[0,1]$, with the value of $f_{A}(x)$ at $x$ representing the "grade of membership» of $x$ in $A$.

Cette définitition fait l'objet de la part de Zadeh de deux précisions importantes, données en notes de bas de page. Premièrement, le domaine de définition de la fonction $f_{A}(x)$ peut être restreint à une partie de $X$. Deuxièmement, on peut généraliser la définition en permettant que la fonction d'appartenance prenne ses valeurs dans un ensemble ordonné convenable, et non nécessairement dans $\mathbf{I}$.

La première remarque est importante parce qu'elle pointe une ambiguïté éventuelle de la théorie des ensembles flous. Au fond, il s'agit de savoir si l'on doit distinguer l'appartenance de degré nul de la non-appartenance, autrement dit de savoir si l'on accepte ou non plusieurs ensembles «vides ». Une autre façon de formuler le problème est de savoir si l'on a affaire à une théorie des ensembles flous ou bien plutôt à une théorie des sous-ensembles flous. De fait ce sont bien des sousensembles flous d'ensembles nets qui ont ainsi été définis, plutôt que des ensembles flous.

La deuxième remarque de Zadeh conduit aux ensembles $L$-flous, ou $L$-ensembles de Goguen [1967], où $L$ désigne un ensemble (partiellement) ordonné, le plus souvent un treillis (complet) complètement distributif (lattice, d'où le $L$ ) :

DÉFINITION 14 (ensembles $L$-flous, ou $L$-ensembles). Un ensemble $L$-flou $\epsilon$ sur un ensemble $X$ est une application $\epsilon: X \rightarrow L$.

Dans la suite, pour tout ensemble $X$, nous noterons $\mathcal{P}_{L}(X)=L^{X}$ l'ensemble des ensembles $L$-flous sur $X$, et pour tout $\epsilon \in \mathcal{P}_{L}(X)$, nous noterons $\operatorname{dom}(\epsilon)$ son domaine, c'est-à-dire $X$. Le support d'un tel ensemble flou $\epsilon$ est défini par

$$
\operatorname{supp}(\epsilon)=\{x \in \operatorname{dom}(\epsilon), \epsilon(x)>0\} .
$$

Notons qu'en remplaçant I par $L$, on sort du domaine d'intuition initial du flou pour entrer dans d'autres univers de valeurs, exactement comme l'on passe du noir et blanc à la couleur. Goguen [1967] précise au sujet de cette généralisation :

Because of the generality of the mathematical definition, some important applications of fuzzy sets do not involve the intuitive concept of fuzziness at all.

Par ailleurs, notons que dans la définition 14, Goguen parle d'ensembles $L$-flous sur $X$ quand Zadeh utilise dans $X$, ce qui ici pourrait indiquer comme l'amorce d'un mouvement d'autonomisation de ces objets, que viendra formaliser la première 
définition, par Goguen [1974], d'une catégorie d'ensembles flous ${ }^{40}$, par laquelle sont notamment définis les morphismes entre $L$-ensembles : un morphisme $f$ d'un $L$ ensemble $\alpha: X \rightarrow L$ vers un autre $\beta: Y \rightarrow L$ est une application $f: X \rightarrow Y$ telle que

$$
\forall x \in X, \alpha(x) \leq \beta(f(x))
$$

Autrement dit, le degré d'existence de l'image d'un élément est nécessairement supérieur à celui de cet élément. Par ailleurs, $f$ désignant toujours une application d'un ensemble $X$ dans un ensemble $Y$, l'image directe d'un sous-ensemble flou $\epsilon$ de $X$, que nous noterons $f(\epsilon)$, se trouve définie par la formule

$$
\forall y \in Y, f(\epsilon)(y)=\bigvee_{x \in f^{-1}(y)} \epsilon(x)
$$

La condition (12) s'écrit alors simplement

$$
f(\alpha) \leq \beta
$$

\subsection{LE PROBLÈME DE LA NÉGATION}

Qu'est-ce qu'un homme révolté? Un homme qui dit non. Mais s'il refuse, il ne renonce pas : c'est aussi un homme qui dit oui, dès son premier mouvement. Un esclave, qui a reçu des ordres toute sa vie, juge soudain inacceptable un nouveau commandement. Quel est le contenu de ce «non»? Il signifie par exemple, "les choses ont trop duré », "jusque-là oui, au-delà non », "vous allez trop loin », et encore, «il y a une limite que vous ne dépasserez pas ». En somme, ce non affirme l'existence d'une frontière. On retrouve la même idée de limite dans ce sentiment du révolté que l'autre « exagère », qu'il étend son droit au-delà d'une frontière à partir de laquelle un autre droit lui fait face et le limite.

Albert Camus [1951]

On a pu mesurer dans les deux premières sections combien l'opérateur $\neg$ de complémentation jouait un rôle central pour la formalisation des frontières nettes. Constituant un pont entre les deux formulations équivalentes que sont d'une part la considération des structures dialectiques et d'autre part celle des frontières comme lieux, il n'en demeure pas moins absent de la première considérée en elle-même de sorte que sa parfaite adéquation au problème de la formalisation des frontières permet en un sens de l'oublier. Une telle adéquation - qui se manifeste par le fait que l'opérateur frontière exprime en tant que lieux et sans le réduire (puisqu'on peut le retrouver) le couple de « logiques spatiales » constituant la structure dialectique considérée - résulte des propriétés remarquables de cet opérateur $\neg$ notamment en relation avec les opérateurs d'union et d'intersection. Or, ces propriétés ne sont pas

\footnotetext{
${ }^{40}$ Pour les aspects catégoriels des notions considérées ici, nous renvoyons le lecteur à l'appendice, section 5.4.9.
} 
toutes conservées lorsqu'on passe au flou, et la centralité de la complémentation pour la question des frontières s'annonce alors plus problématique que dans le cas net. Pour préciser ce point, commençons par remarquer que, dans le cas net, la complémentation ensembliste $\neg$ sur l'ensemble des parties $\mathcal{P}(X)=\mathcal{P}_{\mathbf{2}}(X)$ d'un ensemble $X$ est le prolongement naturel au treillis $\mathbf{2}^{X}$ de l'opérateur classique de la négation logique $\neg$ définie sur le treillis booléen $\mathbf{2}=\{\perp, \top\}$. De ce point de vue, la situation est analogue dans le cas flou : étant donné un ensemble partiellement ordonné $L$, tout opérateur $\neg: L \rightarrow L$, se prolonge naturellement, pour tout ensemble $X$, en un opérateur, noté encore $\neg$, de $\mathcal{P}_{L}(X)$ dans lui-même, en posant simplement

$$
\forall \epsilon \in \mathcal{P}_{L}(X), \neg \epsilon=\neg \circ \epsilon .
$$

La question d'une définition de la complémentation floue se ramène ainsi à celle de la négation floue $\neg: L \rightarrow L$. Dans le cas le plus fréquent où $L$ est un treillis complet complètement distributif, au moins deux conceptions différentes de la négation trouvent à s'opposer. D'une part la négation usuelle en théorie des ensembles flous, généralisation de celle introduite par Zadeh, définie comme involution décroissante du treillis $L$, notée $\neg$; d'autre part la négation dans $L$ en tant qu'algèbre de Heyting, que nous noterons $\sim$, et qui n'est pas, en général, involutive ${ }^{41}$. La négation dans une algèbre de Heyting permet de formaliser la logique intuitioniste, qui respecte le principe de non-contradiction

$$
\forall v \in L, v \wedge \tilde{\neg} v=0 .
$$

Par contre, la logique intuitioniste ne respecte pas le principe du tiers-exclu, en ce sens que

$$
v \vee \tilde{\neg} v=1
$$

n'y est pas toujours vérifié. Ce trait pourrait d'ailleurs contribuer à expliquer une certaine forme de rejet historique vis-à-vis de la logique intuitioniste. Remarquons que la logique du flou est à cet égard dans une bien moins bonne position encore que la logique intuitioniste, puisqu'aucune des deux propriétés rappelées n'est cette fois vérifiée. Ces défaillances de la négation floue, et par conséquent de la complémentation floue, se retrouvent ainsi au cœur du problème de la formalisation des frontières floues comme lieux. En fait, les deux thèmes des frontières et de la négation sont étroitement liés, et de même qu'il est délicat de déterminer les contours précis du concept de frontière, il reste toujours plus facile de dire ce que la négation n'est pas que de dire ce qu'elle est. La définition qui suit ${ }^{42}$ prend acte en tout cas de la spécificité de la négation floue.

DÉFINITION 15 (Treillis flous). Un treillis flou $(L, \leq, 0,1, \vee, \wedge, \neg)$ est un treillis complet, complètement distributif, muni d'une involution décroissante $\neg$.

Si $L$ est un treillis flou et $X$ un ensemble, alors $\mathcal{P}_{L}(X)$ est un treillis flou, dont les opérateurs seront notés comme ceux de $L$.

\footnotetext{
${ }^{41}$ Cf. par exemple [Mac Lane et Moerdijk, 1991, p. 52].

${ }^{42}$ Attribuée par Šstak [1989] à Hutton [1980].
} 


\subsection{TOPOLOGIES FLOUES}

De même que les frontières classiques se rapportent à la topologie, la formalisation des frontières floues s'est le plus souvent déroulée dans le contexte de la topologie floue. La première définition d'une topologie floue sur un ensemble est posée par l'Américain Chang [1968], dans le cas où $L=\mathbf{I}$.

DÉfinition 16 (Topologie floue sur un ensemble). Soit $X$ un ensemble. Une topologie $L$-floue sur $X$ est un ensemble $\mathcal{T} \in \mathcal{P}\left(\mathcal{P}_{L}(X)\right.$ ), stable par suprema (unions floues) quelconques et par infima (intersections floues) finies. Les parties floues de $X$ qui appartiennent à $\mathcal{T}$ sont les ouverts; les parties floues de $X$ de la forme $\neg \Omega$ avec $\Omega \in \mathcal{T}$ sont les fermés.

Šostak [1989] attribue à Goguen [1967] cette généralisation L-floue de la définition de Chang [1968] mais, outre l'antériorité de l'article de Goguen, nous n'avons pas trouvé dans ce dernier de mention explicite de la topologie floue. En remarquant que, dans la définition précédente, on peut en fait se passer de $X$ et de ses points pour s'appuyer sur la seule donnée du treillis flou $\mathcal{P}_{L}(X)$, on obtient la définition suivante.

DÉFINITION 17 (Topologie d'ouverts dans un treillis). Une topologie (d'ouverts) dans ${ }^{43}$ un treillis est une partie de ce treillis, stable par suprema quelconques et par infima finis.

Bien entendu, on peut poser symétriquement :

DÉFINITION 18 (Topologie de fermés dans un treillis). Une topologie de fermés dans un treillis est une partie de ce treillis, stable par infima quelconques et par suprema finis.

Ainsi, une topologie floue sur un ensemble est-elle une topologie dans le treillis des sous-ensembles flous de cet ensemble. Notons que, dans la définition précédente, une topologie dans un treillis est une partie nette de ce treillis ${ }^{44}$.

\subsection{HISTOIRE DES FRONTIÈRES FLOUES}

\subsubsection{Préhistoire des frontières floues}

Les frontières floues, c'est d'abord l'absence de frontières bien définies. Goguen [1967] qualifie ainsi intuitivement un ensemble flou : « a set without a well-defined boundary ». De cette absence naissent les frontières floues, évoquées par Chang [1968] mais également comme notion uniquement intuitive, lorsqu'il écrit : « a fuzzy set $A$ in $X$ is a "class" with fuzzy boundaries ». Nous avons vu comment la notion de frontière débouche nécessairement sur le flou, nous voyons à présent combien la notion de flou est liée dans son intuition originelle à celle de frontière.

\footnotetext{
${ }^{43}$ L'usage pour ce type de généralisation est de conserver la préposition sur, mais cet usage induit une confusion gênante lorsque que l'on veut considérer aussi des topologies sur l'ensemble sous-jacent d'un treillis flou. De plus, la préposition dans semble parfaitement indiquée.

${ }^{44}$ Ce qui n'est pas le cas pour la topologie de Šostak [1985]. Cf. la section 3.4.9., p. 126.
} 
Il est facile de décalquer dans le contexte flou la notion topologique classique de frontière : appelons ainsi frontière floue naïve sur un ensemble $X$ muni d'une topologie floue l'opérateur, que nous noterons $\psi_{0}$, définie par

$$
\forall \epsilon \in \mathcal{P}_{L}(X), \psi_{0}(\epsilon)=\alpha(\epsilon) \wedge \alpha(\neg \epsilon)
$$

où $\alpha$ est l'opérateur sur $\mathcal{P}_{L}(X)$ qui à toute partie floue associe le plus petit fermé la contenant. Comment comprendre que Chang n'ait pas posé cette définition qui pourrait pourtant sembler s'imposer d'elle-même? Cela s'explique probablement par le fait que certaines propriétés fondamentales des frontières topologiques nettes cessent d'être vérifiées par l'opérateur $\psi_{0}$. On constate par exemple que la donnée de cet opérateur ne permet pas de retrouver la topologie floue à laquelle il se trouve associé $^{45}$. À ce stade, le principal problème de la formalisation topologique des frontières floues est ainsi de choisir les propriétés des frontières nettes qu'il s'agit de maintenir et celles auxquelles on accepte de renoncer. Après l'article de Chang, il faut attendre près de dix ans pour que les premières propositions de définitions précises des frontières floues soient publiées, d'une part par les Chinois Liu Ying-ming et Pu Pao-ming, d'autre part par l'Américain Richard Warren. Et leurs définitions sont différentes.

\subsubsection{Frontière de Liu Ying-ming et Pu Pao-ming}

En 1976, Liu Ying-ming et Pu Pao-ming rédigent, en chinois, un article qui propose de prendre ce que nous avons appelé ci-dessus l'opérateur frontière floue naïve $\psi_{0}$ comme définition de la frontière floue d'une partie floue d'un espace topologique flou. Les auteurs signalent qu'avec une telle définition l'union d'une partie avec sa frontière ne coïncide pas, en général, avec son adhérence. Ce fait est attribué à la différence entre le net et le flou, et il n'est pas envisagé qu'une définition différente des frontières puisse préserver cette propriété. Publié en 1977 dans une revue chinoise, cet article sera ensuite traduit en américain et publié trois ans plus tard dans une revue internationale [Liu et $\mathrm{Pu}, 1980$ ], mais la définition des frontières floues de Warren, publiée entre-temps, n'y sera pas mentionnée. À notre connaissance, la frontière de Liu et $\mathrm{Pu}$, autrement dit la frontière floue naïve, ne sera pas utilisée avant les travaux de Kainz et Tang [2002].

\subsubsection{Frontière de Warren}

On doit à Warren [1977] la première publication dans une revue internationale d'une définition précise pour les frontières floues. Nous noterons $\psi_{W}$ l'opérateur frontière qu'il associe à un espace topologique flou. En notant $\alpha$ la fermeture associée à la topologie floue considérée, et notant toujours $\psi_{0}$ l'opérateur « frontière floue naïve » défini ci-dessus, la définition posée par Warren est équivalente à celle-ci :

\footnotetext{
${ }^{45}$ On trouve par exemple facilement une topologie non discrète conduisant au même $\psi_{0}$ que la topologie discrète.
} 
DÉFInITIOn 19. Soit $\epsilon$ une partie d'un espace topologique flou $(X, \mathcal{T})$. La frontière floue (de Warren) de $\epsilon$, notée $\psi_{W}(\epsilon)$, est définie comme l'infimum de tous les ensembles flous fermés $\delta$ dans $X$ tels que $: \delta(x) \geq \alpha(\epsilon)(x)$ pour tout $x \in X$ pour lequel $\psi_{0}(\epsilon)(x)>0$.

Remarquons que cette définition, proposée par Warren dans le seul cadre du flou classique $(L=\mathbf{I})$, s'étend sans difficulté au cas d'un treillis flou quelconque $L$. A cet effet, désignons par $\rho$ l'opérateur défini sur $\mathcal{P}_{L}(X)$ par

$$
\forall \epsilon \in \mathcal{P}_{L}(X), \rho(\epsilon)=1_{\text {supp }(\epsilon)}
$$

L'opérateur $\rho$ est la fermeture associée à la topologie floue sur $X$ pour laquelle les ouverts (et les fermés) sont exactement les parties nettes de X. Nous l'appellerons adhérence de rehaussement sur $X$.

L'opérateur $\psi_{W}$ peut alors s'écrire

$$
\psi_{W}=\alpha(\alpha \wedge \rho \alpha \neg) .
$$

Vérifiant

$$
\alpha=I d \vee \psi_{W}
$$

et

$$
\alpha=\alpha^{c} \vee \psi_{W},
$$

la frontière de Warren caractérise la fermeture $\alpha$, donc les fermés (parties floues de $X$ stables par $\alpha$ ), donc les ouverts. La présence de $\rho$ fait qu'elle n'est pas symétrique : en général, $\psi_{W} \neq \psi_{W}$. Dans le cas où $L=\mathbf{2}, \rho$ est l'adhérence discrète, et la formule (17) équivaut alors à la formule (4). Les opérateurs frontière de la forme $\psi_{W}$, dont Warren propose une caractérisation directe, possèdent de nombreuses propriétés intéressantes, en particulier

$$
\left\{\begin{array}{l}
\psi_{W}(0)=\psi_{W}(1)=0 \\
\forall(\epsilon, \gamma) \in \mathcal{P}_{L}(X)^{2}, \psi_{W}(\epsilon) \leq \epsilon \vee \gamma \vee \psi_{W}(\epsilon \vee \gamma) \\
\forall(\epsilon, \gamma) \in \mathcal{P}_{L}(X)^{2}, \psi_{W}(\epsilon \vee \gamma) \leq \psi_{W}(\epsilon) \vee \psi_{W}(\gamma) \\
\psi_{W} \circ \psi_{W} \leq \psi_{W}
\end{array}\right.
$$

Comme pour l'opérateur $\psi_{0}$, on constate cependant que dans le cas de la topologie discrète, pour laquelle l'adhérence associée $\alpha$ est l'identité de $\mathcal{P}_{L}(X)$, les frontières ne sont généralement pas vides. Plus généralement, une partie à la fois ouverte et fermée peut avoir une frontière de Warren non vide. Ces inconvénients disparaîtront avec l'opérateur frontière introduit par $\mathrm{Pu} \mathrm{Si}$-li [1982] et également considéré, plus tard mais indépendamment, par Cuchillo-Ibanez \& Tarrés [1997].

\subsubsection{Frontière de PuSi-li}

Publié en 1982 dans la revue chinoise Fuzzy mathematics ${ }^{46}$, A better definition of a boundary of a fuzzy set de Pu Si-li [1982] propose de substituer à la frontière $\psi_{W}$ de

\footnotetext{
${ }^{46}$ Cette revue n'est pas référencée dans le catalogue collectif des bibliothèques mathématiques de France, mais la bibliothèque mathématique de Jussieu-Chevaleret en possède néanmoins quelques numéros.
} 
Warren un nouvel opérateur, que nous appellerons frontière de $\mathrm{Pu}$ Si-li et noterons $\psi_{P}$. Écrit en chinois, cet article fait heureusement l'objet d'un résumé en anglais contenant la définition et les principales propriétés de $\psi_{P}$. La définition de Pu Si-li est donnée dans le cas des ensembles flous classiques $(L=\mathbf{I})$ :

DÉFInItion 20. Soit $\epsilon$ une partie d'un espace topologique flou $(X, \mathcal{T})$, de fermeture $\alpha$. La frontière floue (de $\mathrm{Pu} \mathrm{Si}-\mathrm{li})$ de $\epsilon$, notée $\psi_{P}(\epsilon)$, est définie comme l'infimum de tous les ensembles flous fermés $\delta$ dans $X$ tels que : $\delta(x) \geq \alpha(\epsilon)(x)$ pour tout $x \in B_{\alpha}(\epsilon)$, avec

$$
B_{\alpha}(\epsilon)=\{x \in X, \alpha(\epsilon)(x)+\alpha(\neg \epsilon)(x)>1\} .
$$

On a $B_{\alpha}(\epsilon)=\{x \in X, \alpha(\epsilon)(x)>\neg \alpha(\neg \epsilon)(x)\}$. En posant

$$
B_{\alpha}(\epsilon)=1_{\left\{x \in X, \alpha(\epsilon)(x)>\alpha^{c}(\epsilon)(x)\right\}}
$$

on définit un opérateur $B_{\alpha}: \mathcal{P}_{L}(X) \rightarrow \mathcal{P}_{L}(X)$ permettant de prolonger la définition précédente au cas d'un treillis flou $L$ quelconque selon la formule

$$
\psi_{P}=\alpha\left(\alpha \wedge B_{\alpha}\right)
$$

Comme la frontière de Warren, la frontière de $\mathrm{Pu}$ Si-li vérifie

$$
\alpha=I d \vee \psi_{P},
$$

et

$$
\alpha=\alpha^{c} \vee \psi_{P},
$$

elle détermine donc la topologie floue de fermeture $\alpha$. Elle n'est pas symétrique et, dans le cas où $L=\mathbf{2}, \psi_{P}=B_{\alpha} \subset \alpha$ est la frontière topologique associée à $\alpha$. Les opérateurs frontière de la forme $\psi_{P}$ vérifient toutes les propriétés intéressantes des frontières de Warren, en particulier

$$
\left\{\begin{array}{l}
\psi_{P}(0)=\psi_{P}(1)=0 \\
\forall(\epsilon, \gamma) \in \mathcal{P}_{L}(X)^{2}, \psi_{P}(\epsilon) \leq \epsilon \vee \gamma \vee \psi_{P}(\epsilon \vee \gamma) \\
\forall(\epsilon, \gamma) \in \mathcal{P}_{L}(X)^{2}, \psi_{P}(\epsilon \vee \gamma) \leq \psi_{P}(\epsilon) \vee \psi_{P}(\gamma) \\
\psi_{P} \circ \psi_{P} \leq \psi_{P}
\end{array}\right.
$$

mais, contrairement à la frontière de Warren, la topologie discrète donne ici des frontières vides. Plus généralement, une partie à la fois ouverte et fermée a une frontière de $\mathrm{Pu}$ Si-li vide. La frontière de $\mathrm{Pu}$ Si-li améliore donc effectivement celle de Warren, résolvant parfaitement le problème de la formalisation des frontières topologiques floues d'une partie floue d'un ensemble. Par contre, nous verrons que la formule (21) est inadaptée au cadre plus général des frontières prétopologiques floues. 


\subsubsection{Frontières floues de Dubois et Prade}

Twofold Fuzzy Sets, l'article de Dubois et Prade [1981] est publié en 1983, également dans Fuzzy mathematics. Cet article ne se place pas dans l'optique de Warren ou de PuSi-li, qui n'y sont pas cités. Dans le sous-titre de l'article, An approach to the representation of sets with fuzzy boundaries based on possibility and necessity measures, les frontières floues sont d'abord une notion intuitive première. Il s'agit en effet ici non pas de proposer une définition de la frontière floue des ensembles flous, mais de définir de nouveaux ensembles flous - les ensembles flous à double-feuillets - à partir d'une idée intuitive des frontières, quitte à définir ensuite la frontière floue de tels ensembles. Ainsi, la frontière de Dubois et Prade n'est pas d'abord un lieu, mais la donnée de deux lieux flous, l'adhérence floue et l'intérieur flou d'un ensemble non défini en dehors de cette donnée. $L$ désignant un treillis complet, on pose plus précisément les définitions suivantes.

DÉfinition 21 (Cœur d'un $L$-ensemble). Soit $\epsilon \in \mathcal{P}_{L}(X)$ une partie $L$-floue de $X$. Le cour de $\epsilon$ est

$$
\kappa(\epsilon)=1_{\{x \in X, \epsilon(x)=1\}}
$$

DÉFInItion 22 (Ensembles à double-feuillets flous). Étant donné X un ensemble, un ensemble de Dubois et Prade, ou ensemble à double-feuillets flous sur $X$ est un couple $\tilde{\epsilon}=(\iota, \alpha) \in \mathcal{P}_{L}(X)^{2}$, avec

$$
\operatorname{dom}(\iota)=\operatorname{dom}(\alpha)=X
$$

et tel que

$$
\iota \leq \kappa(\alpha)
$$

Le domaine de $\tilde{\epsilon}$ est $\operatorname{dom}(\tilde{\epsilon})=X$. On appelle intérieur de $\tilde{\epsilon}$ sa première composante $\iota(\tilde{\epsilon})=\iota_{\tilde{\epsilon}}=\iota$ et adhérence de $\tilde{\epsilon}$ sa deuxième composante $\alpha(\tilde{\epsilon})=\alpha_{\tilde{\epsilon}}=\alpha$. Un morphisme d'ensembles à double-feuillets flous $f: \tilde{\epsilon} \rightarrow \tilde{\gamma}$ est une application du domaine de $\tilde{\epsilon}$ dans le domaine de $\tilde{\gamma}$ telle que $f$ soit une application floue de l'intérieur (resp. l'adhérence) de $\tilde{\epsilon}$ dans l'intérieur (resp. l'adhérence) de $\tilde{\gamma}$, autrement dit (d'après la formule (14)) telle que

$$
f\left(\iota_{\tilde{\epsilon}}\right) \leq \iota_{\tilde{\gamma}} \text { et } f\left(\alpha_{\tilde{\epsilon}}\right) \leq \alpha_{\tilde{\gamma}}
$$

On note $\mathcal{F F}_{L}(X)$, ou simplement $\mathcal{F} \mathcal{F}(X)$, le treillis des ensembles à doublefeuillets flous de domaine $X$ pour l'ordre, noté $\leq_{\mathcal{F} \mathcal{F}}$, induit par l'inclusion $\mathcal{F} \mathcal{F}(X) \subset$ $\mathcal{P}_{L}(X)^{2}$.

Le point de vue dialectique que nous avons développé dans le cas des frontières nettes se trouve conforté dans le domaine flou par le choix de Dubois et Prade d'identifier la donnée d'une frontière floue avec celle d'un couple intérieur/adhérence. Il n'en demeure pas moins que ce sont bien des ensembles flous qui sont ainsi définis, plutôt que des frontières. Cette distinction se manifeste en particulier par l'ordre sur le treillis $\mathcal{F} \mathcal{F}(X)$, défini par la formule

$$
\tilde{\epsilon}_{1} \leq \leq_{\mathcal{F F}} \tilde{\epsilon}_{2} \Longleftrightarrow\left\{\begin{array}{l}
l_{\tilde{\epsilon}_{1}} \leq \iota_{\tilde{\epsilon}_{2}} \\
\alpha_{\tilde{\epsilon}_{1}} \leq \alpha_{\tilde{\epsilon}_{2}}
\end{array}\right.
$$


à distinguer de l'ordre dialectique ${ }^{47}$

$$
\tilde{\epsilon}_{1} \preceq \tilde{\epsilon}_{2} \Longleftrightarrow\left\{\begin{array}{l}
\iota_{\tilde{\epsilon}_{1}} \geq \iota_{\tilde{\epsilon}_{2}} \\
\alpha_{\tilde{\epsilon}_{1}} \leq \alpha_{\tilde{\epsilon}_{2}}
\end{array}\right.
$$

qui, dans le cas présent, ne conduit d'ailleurs pas à un treillis.

PROPOSITION 15. $\mathcal{F} \mathcal{F}(X)=\mathcal{P}_{L_{2}}(X)$, où $L_{2}=\mathcal{F} \mathcal{F}(\{*\})$.

DÉMONSTRATION. La condition $\iota \leq \kappa(\alpha)$ est vérifiée si et seulement si elle est vérifiée localement.

On a

$$
\begin{aligned}
\mathcal{F F}(\{*\}) & =\left\{(\iota, \alpha) \in L^{2}, \alpha<1 \Rightarrow \iota=0\right\} \\
& =\{0\} \times(L \backslash\{1\}) \cup L \times\{1\}
\end{aligned}
$$

et puisque pour tout $(\iota, \alpha) \in L^{2},(0, \alpha) \leq(\iota, 1)$, l'ordre induit sur $\mathcal{F} \mathcal{F}(\{*\})$ par le treillis $L^{2}$ fait de $L_{2}$ un treillis isomorphe à celui obtenu en « recollant » l'un à la suite de l'autre deux treillis isomorphes à $L$. Dans le cas où $L$ possède une propriété d'auto-similarité adéquate, on obtient alors $L_{2} \simeq L$. C'est notamment le cas pour le treillis des valeurs floues classiques $L=\mathbf{I}$, l'isomorphisme de treillis le plus simple que l'on peut imaginer dans ce cas étant $\theta: L \rightarrow L_{2}$, défini pour tout $\epsilon \in \mathbf{I}$ par

$$
\theta(\epsilon)=(\iota, \alpha) \text { avec }\left\{\begin{array}{l}
\alpha=\min (1,2 \epsilon) \\
\iota=\max (0,2 \epsilon-1)
\end{array}\right.
$$

Ces mêmes formules s'étendent aux treillis $\mathcal{P}_{\mathbf{I}}(X)$ et $\mathcal{F} \mathcal{F}(X)$, définissant un isomorphisme du premier sur le second, que nous noterons encore $\theta$. Un tel isomorphisme conduit naturellement à associer à tout $\epsilon \in \mathcal{P}_{\mathbf{I}}(X)$ les sous-ensembles flous $\alpha_{\theta}(\epsilon)$ et $\iota_{\theta}(\epsilon)$, respectivement définis par

$$
\alpha_{\theta}(\epsilon)=\alpha(\theta(\epsilon))=\min (1,2 \epsilon)
$$

et

$$
\iota_{\theta}(\epsilon)=\iota(\theta(\epsilon))=\max (0,2 \epsilon-1) .
$$

Or, $\iota_{\theta} \leq I d \leq \alpha_{\theta}$ et $\iota_{\theta}=\neg \alpha_{\theta} \neg=\alpha_{\theta}{ }^{c}$, de sorte que le couple d'opérateurs $\left(\iota_{\theta}, \alpha_{\theta}\right)$ de $\mathcal{P}_{\mathbf{I}}(X)$ constitue ce que nous appellerons une structure dialectique floue symétrique sur $X$ (cf. la section 3.6., p. 130).

Revenons à l'article de Dubois et Prade [1981]. Une notion de frontière floue y est définie pour les ensembles à double-feuillets flous.

DÉFInition 23. Soit $\tilde{\epsilon} \in \mathcal{F F}_{\mathbf{I}}(X)$ un ensemble à double-feuillets flous. La frontière de Dubois et Prade de $\tilde{\epsilon}$ est $\Psi_{0}(\tilde{\epsilon})=\alpha(\tilde{\epsilon}) \wedge \neg \iota(\tilde{\epsilon}) \in \mathcal{P}_{\mathbf{I}}(X)$.

\footnotetext{
${ }^{47}$ Cf. la section 3.6 .
} 
Ici, à un ensemble indéfini en dehors de ses frontières intérieure et extérieure est associée une frontière, floue au sens classique, et cette démarche se situe a priori en dehors de toute considération topologique. Dubois et Prade développent si l'on peut dire une topologie en l'absence du sous-ensemble concerné. De notre point de vue, les définitions de Dubois et Prade sont intéressantes parce qu'elle sont structurellement « dialectiques », au sens où intériorité et adhérence y sont découplées (dans le moment même où on en forme le couple). Mais comment relier cette définition à celles dont nous disposons jusqu'à présent? Le fait que l'opérateur $\Psi_{0}$ ne donne pas des frontières de même nature que les ensembles à double-feuillets auxquels il s'applique distingue absolument cette définition de toutes celles considérées jusque ici. Utilisons l'isomorphisme $\theta$, par lequel les double-feuillets de Dubois et Prade ont déjà conduit à une structure dialectique floue intéressante, cette fois pour définir un opérateur frontière $\psi_{1}: \mathcal{P}_{\mathbf{I}}(X) \rightarrow \mathcal{P}_{\mathbf{I}}(X)$. Pour cela, posons

$$
\psi_{1}=\Psi_{0} \circ \theta \text {. }
$$

Définie en dehors de considérations topologiques, $\psi_{1}$ est topologiquement discrète sur les ensembles nets. Directement issue des définitions de Dubois \& Prade, elle constitue un exemple intéressant de frontière floue.

PROPOSITION 16. $\psi_{1}$ n'est pas une frontière de Warren, ni une frontière de Pu Si-li.

DÉmonstration. Vérifions-le d'abord dans le cas de $X=\{*\}$. La fermeture $\alpha$ associée à une topologie floue sur $X$ est une application croissante de $\mathbf{I}$ dans luimême, telle que $\alpha(0)=0, \alpha \geq I d$ et $\alpha \circ \alpha=\alpha$. Si $\epsilon<1$, on a $\neg \epsilon>0 \Rightarrow \alpha \neg \epsilon>0$ d'où $\rho \alpha \neg \epsilon=1$ et $\psi_{W}(\epsilon)=\alpha(\epsilon)$. Par ailleurs, si $\psi_{P}(\epsilon) \neq 0$, alors $B_{\alpha}(\epsilon)=1$, d'où $\psi_{P}(\epsilon)=\alpha(\epsilon) \geq \epsilon$. On vérifie enfin que $\psi_{1}=2(\operatorname{Id} \wedge \neg)$ de sorte que, par exemple

$$
\psi_{1}\left(\frac{3}{4}\right)=\frac{1}{2}
$$

tandis que $\psi_{W}\left(\frac{3}{4}\right)=\alpha\left(\frac{3}{4}\right) \geq \frac{3}{4}$ et que $\psi_{P}\left(\frac{3}{4}\right)$ est soit nul, soit $\geq \frac{3}{4}$, mais ne saurait être égal à $\frac{1}{2}$.

En remarquant que $\psi_{1}$ est définie localement, on étend la démonstration à tout $X$.

\subsubsection{GF-Frontière d'Adnadjevič}

Avec Adnadjevič [1986], la frontière devient un objet dans une catégorie où l'on dispose de structures floues particulières faisant appel à une variété de treillis de valeurs, et où est définie, pour chaque objet, une structure dialectique topologique dans le treillis « varié » des sous-ensembles flous de cet objet. Il s'agit donc d'une généralisation originale des espaces topologiques flous [Chang, 1968], à la fois en terme d'enrichissement des structures floues et de passage du topologique au dialectique topologique. Contrairement aux démarches de Warren et $\mathrm{Pu} \mathrm{Si}$-li, les treillis de valeurs considérés ici sont des treillis certes complets, mais ne sont pas des treillis flous : il n'y a pas en général de négation ou de complémentation dans ces treillis 
et, de même que Dubois \& Prade considèrent des couples intérieur/adhérence, la structure dialectique d'Adnadjevič est donnée par un couple formé d'une topologie d'ouverts et d'une topologie de fermés.

DÉFInItion 24. Soit $\left(L, \leq_{L}\right)$ et $\left(G, \leq_{G}\right)$ deux treillis complets. Nous dirons que $G$ est plus grand que $L$, et nous noterons $L \leq G$, si $L \subset G$ et $\leq_{L}=\leq_{G \mid L}$, restriction à $L$ de la relation d'ordre de $G$.

On peut décomposer la définition des objets considérés par Adnadjevič en deux étapes. On munit d'abord un ensemble $X$ d'une famille $\mathcal{L}=\left(L_{x},\right)_{x \in X}$ de treillis complets (non nécessairement flous) d'un certain type, tels que pour tout $x \in X$, $L_{x} \leq I$ et $0_{L_{x}}=0_{I}$. L'ensemble de toutes les familles de ce type constitue un treillis complet ${ }^{48}$. Un espace d'Adnadjevič (ou GF-espace $\left.{ }^{49}\right)(X, \mathcal{L}, \Omega, \Phi)$ est obtenu en munissant ensuite un tel objet flou $(X, \mathcal{L})$ d'un couple $(\Omega, \Phi)$ de topologies dans le treillis

$$
\mathcal{P}_{\mathcal{L}}(X)=\prod_{x \in X} L_{x}
$$

la première, $\Omega \subset \mathcal{P}_{\mathcal{L}}(X)$, donnée par les ouverts, la seconde, $\Phi \subset \mathcal{P}_{\mathcal{L}}(X)$ par les fermés (cf. les définitions 17 et 18, p. 117).

Pour Adnadjevič, la frontière d'un élément de $\mathcal{P}_{\mathcal{L}}(X)$ - donc d'un sous-ensemble flou classique de $X$ - est un espace d'Adnadjevič. Comme pour la frontière de Dubois \& Prade, les frontières d'Adnadjevič ne sont donc pas de même nature que ce dont elles sont la frontière (par contre, avec Adnadjevič, c'est la frontière qui est l'objet mathématique le plus complexe). Notons que la structure dialectique d'un tel espace-frontière $\left(F, \mathcal{K}, \Omega^{\mathcal{K}}, \Phi^{\mathcal{K}}\right)$ y sera non pas induite (structure initiale) par inclusion dans l'espace ambiant $(X, \mathcal{L}, \Omega, \Phi)$, mais plutôt projetée (structure finale) avec une projection caractérisée par une application $\sigma: \mathcal{P}_{\mathcal{L}}(X) \rightarrow \mathcal{P}_{\mathcal{K}}(F)$ définie grâce à des propriétés de convexité propres au type de treillis $L_{x}$ utilisés par Adnadjevič.

Étant donné un GF-espace $(X, \mathcal{L}, \Omega, \Phi)$, en notant, pour tout sous-espace $(F, \mathcal{K})$, $\sigma$ l'application mentionnée ci-dessus, $\iota$ l'opérateur d'ouverture associé à la topologie d'ouverts $\Omega$, et $\alpha$ l'opérateur de fermeture associé à la topologie de fermés $\Phi$, la frontière d'Adnadjevič de $\epsilon \in \mathcal{P}_{\mathcal{L}}(X)$ s'écrit $\operatorname{Fr}(\epsilon)=\left(F, \mathcal{K}, \Omega^{\mathcal{K}}, \Phi^{\mathcal{K}}\right)$ où, ayant posé pour tout $x \in X$

$$
K_{x}=\left\{t \in L_{x}, \iota(\epsilon)(x)<t \leq \alpha(\epsilon)(x)\right\}
$$

on prend $F=\left\{x \in X, K_{x} \neq \emptyset\right\}$, puis $\mathcal{K}=\prod_{x \in F}\left(\{0\} \cup K_{x} \cup\{1\}\right)$ et enfin $\Omega^{\mathcal{K}}=$ $\sigma(\Omega)$ et $\Phi^{\mathcal{K}}=\sigma(\Phi)$. Remarquons que, dans le cas où $\iota=\neg \alpha \neg=\alpha^{c}$, le support $F$ de $\operatorname{Fr}(\epsilon)$ coïncide avec celui de l'ensemble flou $B_{\alpha}(\epsilon)$ qui apparaît dans la frontière de Pu Si-li (formule (20), p. 120).

\footnotetext{
${ }^{48}$ Cette première étape correspond ainsi à ce que nous appelons en section 5.3. une catégorie à treillis de structures.

${ }^{49} \mathrm{GF}$ pour Generalised Fuzzy.
} 


\subsubsection{Frontière de LiuXiao-shi}

Une définition de la frontière d'un élément d'un treillis moléculaire topologique est donnée par Liu Xiao-shi [1987] dans un court article de trois pages publié en chinois dans la revue chinoise Kexue Tongbao. Selon Wu Guang-qing et Zheng Chong-you [1987], la définition de Liu Xiao-shi est identique à la leur (cf. ci-dessous), sauf en ce qui concerne la notion d'intérieur employée.

\subsubsection{Frontière de Wu Guang-qing et Zheng Chong-you}

Publié en 1991, l'article de Wu Guang-qing et Zheng Chong-you [1987] se place, comme celui de Liu Xiao-shi, dans le cadre d'un treillis flou quelconque ${ }^{50}$. Un tel treillis $\mathcal{P}$ étant muni d'une topologie d'ouverts ${ }^{51} \Omega$, nous noterons $\alpha$ l'opérateur adhérence associé, et $\iota$ l'opérateur intérieur ${ }^{52}$. La définition de l'opérateur frontière de Wu Guang-qing et Zheng Chong-you, $\psi_{W Z}: \mathcal{P} \rightarrow \mathcal{P}$, fait appel aux molécules.

DÉFinition 25 (Molécules). L'ensemble $M_{\mathcal{P}}$ des molécules d'un treillis complet $\mathcal{P}$ est l'ensemble des $m \in \mathcal{P}$, non nuls, tels que pour tous $s_{1}$ et $s_{2}$ dans $\mathcal{P}, m \leq s_{1} \vee s_{2}$ implique $\left(m \leq s_{1}\right.$ ou $\left.m \leq s_{2}\right)$.

DÉfinition 26. Pour tout $\epsilon \in \mathcal{P}$, on appelle ensemble des points frontières de $\epsilon$ et l'on note $[\epsilon]$ l'ensemble

$$
[\epsilon]=\left\{a \in M_{\mathcal{P}}, a \leq \alpha(\epsilon) \text { et } a \not \leq \iota(\epsilon)\right\} .
$$

La frontière de Wu Guang-qing et Zheng Chong-you est alors définie par

$$
\psi_{W Z}(\epsilon)=\bigvee[\epsilon]
$$

Exemples.

1. Soit $L$ un treillis flou, $X$ un ensemble et $\Omega$ une topologie dans le treillis flou $\mathcal{P}=\mathcal{P}_{L}(X)$. La relation d'ordre de $\mathcal{P}$ découlant ponctuellement de celle de $L$, les molécules de $\mathcal{P}$ sont les $\epsilon: X \rightarrow L$ dont le support est réduit à un point de $X$ et dont la valeur en ce point est une molécule de $L$. Nous noterons $\beta_{x}$ la molécule de support $\{x\}$ et de valeur $\beta$ en $x$.

2. On suppose à présent $L=I$. Puisque $\left.\left.M_{L}=\right] 0,1\right]$, on a $\left.\left.M_{\mathcal{P}} \simeq X \times\right] 0,1\right]$, d'où

$$
\begin{aligned}
{[\epsilon] } & =\left\{\beta_{x} \in M, \beta_{x} \leq \alpha(\epsilon)(x) \text { et } \beta_{x} \not \leq \iota(\epsilon)(x)\right\} \\
& =\left\{\beta_{x} \in M, \iota(\epsilon)(x)<\beta_{x} \leq \alpha(\epsilon)(x)\right\} .
\end{aligned}
$$

De la formule (20) page 120, on déduit que, pour tout $x \in X$, on a soit $B_{\alpha}(\epsilon)(x)=0$ et dans ce cas $\psi_{W Z}(\epsilon)(x)=0$, soit $B_{\alpha}(\epsilon)(x)=1$, et dans ce cas $\psi_{W Z}(\epsilon)(x)=\alpha(\epsilon)(x)$, de sorte que

$$
\psi_{W Z}=\alpha \wedge B_{\alpha} .
$$

\footnotetext{
${ }^{50} \mathrm{Il}$ n'est donc pas fait appel à un ensemble de points, le treillis flou considéré généralisant celui des parties floues d'un tel ensemble.

${ }^{51}$ Cf. la définition 17, p. 117.

${ }^{52} \alpha$ est donc défini comme l'inf des majorants fermés et $\iota$ comme le sup des minorants ouverts des éléments auxquels ces opérateurs sont appliqués.
} 
D'après la formule (21) page 120, on a ainsi dans ce cas

$$
\psi_{P}=\alpha \circ \psi_{W Z}
$$

À part le fait que $\psi_{W Z}$ ne conduit pas en général à des frontières fermées (ce qui cesse d'ailleurs d'être une exigence naturelle dans le cadre plus général des structures prétopologiques), les propriétés les plus importantes de $\psi_{P}$ sont conservées : comme les frontières de Warren et de $\mathrm{PuSi}$-li, la frontière de Wu Guang-qing et Zheng Chong-you vérifie notamment

$$
\left\{\begin{array}{l}
\alpha=I d \vee \psi_{W Z}, \\
\alpha=\alpha^{c} \vee \psi_{W Z}, \\
\psi_{W Z}(0)=\psi_{W Z}(1)=0, \\
\forall(\epsilon, \gamma) \in \mathcal{P}^{2}, \psi_{W Z}(\epsilon) \leq \epsilon \vee \gamma \vee \psi_{W Z}(\epsilon \vee \gamma), \\
\forall(\epsilon, \gamma) \in \mathcal{P}^{2}, \psi_{W Z}(\epsilon \vee \gamma) \leq \psi_{W Z}(\epsilon) \vee \psi_{P}(\gamma)
\end{array}\right.
$$

Ainsi, $\psi_{W Z}$ caractérise elle aussi la topologie floue de fermeture $\alpha$, et l'on vérifie qu'elle coïncide avec la frontière topologique associée à $\alpha$ dans le cas où $L=\mathbf{2}$. Comme pour la frontière de $\mathrm{PuSi}$-li, avoir une frontière vide équivaut à être simultanément ouvert et fermé. Par contre, la relation

$$
\psi_{W Z}\left(\psi_{W Z}(\epsilon) \leq \psi_{W Z}(\epsilon)\right.
$$

n'est satisfaite que si $\psi_{W Z}(\epsilon)$ est fermé.

\subsubsection{Frontière fuzzyfiante de Ying Mingsheng}

En 1985, Šostak [1985, 1989] introduit ce qu'il appelle simplement les espaces topologiques flous, qui généralisent les espaces topologiques flous classiques [Chang, 1968]. Partant de la remarque qu'un espace topologique flou au sens de Chang est défini par une partie nette de l'ensemble des parties floues du domaine, il propose qu'il le soit plutôt par une partie $(L-)$ floue. On obtient ainsi une définition topologique qui n'entre pas dans le cadre des topologies dans un treillis (cf. la définition 17, p. 117), toute partie floue de $X$ s'y trouvant affectée d'un certain degré d'ouverture.

DÉFINITION 27 (Espace de Šostak). Un espace topologique de Šostak est un couple $(X, \tau)$, où $X$ désigne un ensemble et $\tau$ un élément du treillis ${ }^{53} \mathcal{P}_{L}\left(\mathcal{P}_{L}(X)\right)$ qui vérifie ${ }^{54}$

$$
\begin{cases}\text { 1. } & \tau(1)=1 \\ \text { 2. } & \forall \tilde{\epsilon} \in \mathcal{P}\left(\mathcal{P}_{L}(X)\right), \bigwedge \tau(\tilde{\epsilon}) \leq \tau(\bigvee \tilde{\epsilon}) \\ \text { 3. } & \forall\left(\epsilon_{1}, \epsilon_{2}\right) \in \mathcal{P}_{L}(X)^{2}, \tau\left(\epsilon_{1}\right) \wedge \tau\left(\epsilon_{2}\right) \leq \tau\left(\epsilon_{1} \wedge \epsilon_{2}\right)\end{cases}
$$

Indépendamment de Šostak, dans le cadre d'une démarche s'appuyant sur un langage formel adapté à la sémantique floue, Ying Mingsheng [1987, 1989] retrouve

\footnotetext{
${ }^{53}$ Une topologie de Šostak est ainsi ce que Goguen [1973] appelle a fuzzy fuzzy set sur $X$.

${ }^{54}$ On notera que la deuxième propriété entraîne notamment $\tau(0)=1$.
} 
ces topologies, qu'il nomme topologies bifloues. Dans le langage formel utilisé par Ying, la propriété 3 ci-dessus s'écrit par exemple

$$
\text { Pour tout }\left(\epsilon_{1}, \epsilon_{2}\right), \vDash\left(\epsilon_{1} \in \tau\right) \wedge\left(\epsilon_{2} \in \tau\right) \longrightarrow \epsilon_{1} \cap \epsilon_{2} \in \tau \text {. }
$$

Parmi les topologies bifloues sur $X$, Ying (M.) considère tout particulièrement celles qu'il appelle fuzzifying topologies, ce que nous traduirons par topologies fuzzyfiantes, c'est-à-dire celles qui appartiennent à $\mathcal{P}_{L}(\mathcal{P}(X))$. Ainsi, une topologie fuzzyfiante indique le degré d'ouverture de toute partie nette de $X$. C'est dans ce cadre particulier que Ying Mingsheng [1989] définit, par une expression du langage formel à sémantique floue mentionné, la frontière floue d'une partie nette. Dans ce langage, cette définition peut s'écrire (en gardant nos notations)

$$
x \in \psi_{Y}(A):=(x \in \alpha(A)) \wedge(x \in \alpha(\neg A)) .
$$

Elle est donc formellement identique à l'opérateur $\psi_{0}$, c'est-à-dire à la définition classique des frontières topologiques. Après retranscription dans le langage mathématique ordinaire, et en prenant pour treillis de base un treillis flou $L$ quelconque, on obtient la définition équivalente suivante.

DÉFInition 28. Soit $(X, \tau)$ un espace topologique L-fuzzyfiant. La frontière de Ying Mingsheng est l'opérateur $\psi_{Y}: \mathcal{P}(X) \rightarrow \mathcal{P}_{L}(X)$ défini pour tout $A \in \mathcal{P}(X)$ et tout $x \in X$ par

$$
x \in A \Rightarrow \psi_{Y}(A)(x)=\bigwedge_{x \in B \subset A} \neg \tau(B)
$$

et

$$
x \in \neg A \Rightarrow \psi_{Y}(A)(x)=\bigwedge_{x \in B \subset \neg A} \neg \tau(B) .
$$

Exprimées dans le langage formel en question, les propriétés de la frontière de Ying sont semblables à celles d'une frontière topologique (on notera en particulier sa symétrie : $\left.\psi_{Y}(A)=\psi_{Y}(\neg A)\right)$. Ce qui fait l'originalité de la frontière de Ying est ce qui fait l'originalité de sa topologie en général, à savoir l'emploi d'une logique floue formalisée pour l'exploration d'espaces de Šostak. On pourrait dès lors, ce que nous ne ferons pas dans cet article, chercher à fuzzyfier de la même manière les frontières dialectiques (nettes).

\subsubsection{L'article de Cuchillo-Ibanez ES Tarrés}

Dans cet article publié en 1997, Cuchillo-Ibanez et Tarrés se proposent d'améliorer la définition de Warren [1977]. Ils retrouvent ainsi, indépendamment de Pu Si-li [1982] dont ils ignorent l'article, la frontière définie antérieurement par le mathématicien chinois.

\subsection{FRONTIÈRES PRÉTOPOLOGIQUES FLOUES}

Comme le montre Robert Badard [1981], la notion d'adhérence prétopologique s'étend immédiatement aux ensembles $L$-flous (où $L$ désigne un treillis flou). 
DÉFinition 29 (Adhérences floues). Soit $X$ un ensemble. Une adhérence (prétopologique) floue sur $X$ est une application $\alpha: \mathcal{P}_{L}(X) \rightarrow \mathcal{P}_{L}(X)$ telle que $\alpha(0)=0$ et

$$
\forall \epsilon \in \mathcal{P}_{L}(X), \epsilon \leq \alpha(\epsilon)
$$

Si de plus a vérifie

$$
\forall(\epsilon, \gamma) \in \mathcal{P}_{L}(X)^{2}, \epsilon \leq \gamma \Rightarrow \alpha(\epsilon) \leq \alpha(\gamma)
$$

nous dirons que $\alpha$ est de type $\mathcal{V}$. Si elle vérifie en outre

$$
\forall(\epsilon, \gamma) \in \mathcal{P}(X)^{2}, \alpha(\epsilon \vee \gamma)=\alpha(\epsilon) \vee \alpha(\gamma)
$$

elle est dite de type $\mathcal{V}_{\mathcal{D}}$. Nous dirons d'une adhérence de type $\mathcal{V}$ qui est idempotente $\left(\alpha^{2}=\alpha\right)$ qu'elle est de type $\mathcal{V}_{\mathcal{I}}$. Nous dirons enfin qu'une adhérence est de type $\mathcal{T}$ (ou topologique ${ }^{55}$ ) si elle est à la fois de type $\mathcal{V}_{\mathcal{I}}$ et $\mathcal{V}_{\mathcal{D}}$.

La définition des espaces prétopologiques flous en découle ${ }^{56}$, tandis que la notion d'image directe d'un sous-ensemble flou par une application, qu'exprime la formule (13), permet de définir les morphismes prétopologiques d'une manière calquée sur celle de la définition 5 :

DÉFINITION 30. Un espace prétopologique flou est un ensemble muni d'une adhérence floue. Si cette adhérence est d'un certain type, nous dirons que l'espace prétopologique est du type en question. Un morphisme prétopologique, ou application continue, d'un espace prétopologique flou $(X, \alpha)$ vers un autre $(Y, \beta)$ est une application $f: X \rightarrow Y$ telle que

$$
\forall \epsilon \in \mathcal{P}_{L}(X), f(\alpha(\epsilon)) \leq \beta(f(\epsilon)) .
$$

Muni de la relation d'ordre dont l'orientation, du discret vers le grossier, est choisie en cohérence avec l'orientation des divers treillis de structures que nous sommes amené à considérer, à savoir

$$
\alpha_{1} \leq \alpha_{2} \Leftrightarrow \forall \epsilon \in \mathcal{P}_{L}(X), \alpha_{1}(\epsilon) \leq \alpha_{2}(\epsilon)
$$

l'ensemble des prétopologies floues (resp. l'ensemble des prétopologies floues de type donné) sur un ensemble $X$ constitue un treillis complet $\mathcal{F} \mathcal{P} a(X)$. L'adhérence minimale est donc l'adhérence discrète (la plus fine), et l'adhérence maximale l'adhérence grossière (la plus «épaisse »).

Remarque. On peut également représenter les structures prétopologiques flous en termes d'intérieurs, c'est-à-dire des opérateurs $\iota$ vérifiant $\iota\left(1_{X}\right)=1_{X}$ et, pour tout

\footnotetext{
${ }^{55}$ Dénomination justifiée par le fait, facilement vérifié, qu'une adhérence prétopologique floue est topologique si et seulement s'il existe une topologie floue dont elle soit l'adhérence.

${ }^{56}$ Nous renvoyons à l'appendice (section 5.4.10.) pour une présentation catégorielle des espaces prétopologiques flous, dont découle immédiatement la possibilité de procéder sur ces espaces à toutes les constructions usuelles (produit cartésien, etc.).
} 
$\epsilon \in \mathcal{P}_{L}(X), \iota(\epsilon) \leq \epsilon$. Le treillis $\mathcal{F} \mathcal{P} i(X)$ des intérieurs sur $X$ doit à son tour être défini par une relation d'ordre $\preceq$ orientée du discret vers le grossier, autrement dit, étant donnés deux opérateurs intérieurs $\iota_{1}$ et $\iota_{2}$ sur $\mathcal{P}_{L}(X)$, telle que

$$
\begin{aligned}
\iota_{1} \preceq \iota_{2} & \Leftrightarrow \forall \epsilon \in \mathcal{P}_{L}(X), \iota_{2}(\epsilon) \leq \iota_{1}(\epsilon) \\
& \Leftrightarrow \quad \iota_{2} \leq \iota_{1},
\end{aligned}
$$

de sorte que l'intérieur minimal est l'intérieur discret et l'intérieur maximal est l'intérieur grossier, c'est-à-dire l'opérateur qui à toute partie floue propre de $X$ associe la partie vide.

Exemple. L'adhérence de rehaussement $\rho: \mathcal{P}_{L}(X) \rightarrow \mathcal{P}_{L}(X)$, définie p. 119 par la formule (16), est une adhérence floue topologique. Plus généralement, toute topologie floue sur un ensemble $X$, dont les fermés constituent un ensemble $\Phi \subset \mathcal{P}_{L}(X)$, est caractérisée par son adhérence floue topologique $\alpha$, définie pour tout $\epsilon \in \mathcal{P}_{L}(X)$ par

$$
\alpha(\epsilon)=\bigwedge_{\epsilon \leq \gamma \in \Phi} \gamma \in \Phi
$$

Dans la section 2.4., nous avons associé à toute adhérence prétopologique nette un opérateur frontière qui caractérise cette adhérence. Si nous voulons faire de même pour les espaces prétopologiques flous, nous pouvons considérer d'abord les différentes frontières proposées dans le cas des espaces topologiques flous. Exprimées en termes d'adhérence, les frontières de Warren, de Pu Si-li et de Wu Guang-qing et Zheng Chong-you se généralisent directement à tout espace prétopologique. Cependant, alors que dans le cas où $\alpha$ est une adhérence floue topologique les trois opérateurs en question caractérisent $\alpha$, il n'en va plus de même en général, seule la frontière de Wu Guang-qing et Zheng Chong-you, définie par

$$
\psi_{W Z}=\alpha \wedge B_{\alpha}
$$

(où $B_{\alpha}(\epsilon)=1_{\left\{x \in X, \alpha(\epsilon)(x)>\alpha^{c}(\epsilon)(x)\right\}}$ et $\alpha^{c}=\neg \alpha \neg$ ) vérifiant encore, lorsque $\alpha$ est une adhérence prétopologique floue quelconque, la relation

$$
\psi_{W Z} \vee I d=\alpha
$$

ainsi d'ailleurs que

$$
\psi_{W Z} \vee \alpha^{c}=\alpha .
$$

La frontière de Wu Guang-qing et Zheng Chong-you s'impose ainsi en prétopologie floue, d'autant que son principal inconvénient dans le cas topologique, celui de conduire à des frontières non nécessairement fermées, cesse d'en être un dans le cas de prétopologies quelconques, l'exigence de fermeture des frontières étant alors le plus souvent excessive, par exemple lorsqu'un espace prétopologique n'admet aucune partie propre fermée ${ }^{57}$.

\footnotetext{
${ }^{57}$ C'est par exemple le cas de l'adhérence définie par la frontière japonaise sur un graphe connexe.
} 


\subsection{FRONTIÈRES L-DIALECTIQUES}

Un objectif légitime de la formalisation des frontières $L$-dialectiques serait que la donnée des frontières comme lieux caractérise une structure dialectique sous-jacente, représentée par un couple de prétopologies floues. L'examen des frontières nettes nous a en effet montré l'importance de la dimension « dialectique » des frontières : celles-ci sont toujours le résultat de la mise en présence de deux « logiques » différentes de l'espace, l'une relative à l'adhérence, et partant, à l'extériorité, l'autre à l'intériorité.

Si ce problème d'une caractérisation des structures dialectiques par les frontières a trouvé des solutions satisfaisantes dans les cas particuliers (mais déjà très généraux) des espaces dialectiques nets ${ }^{58}$ - ce qui inclut bien sûr les espaces topologiques ordinaires - des espaces topologiques flous (frontières de $\mathrm{Pu} \mathrm{Si}$-li) et plus généralement des espaces prétopologiques flous (frontières de Wu Guang-qing et Zheng Chong-you), la situation est différente dans le cas général des structures dialectiques floues, pour lesquelles il faut renoncer à trouver une formule permettant d'associer à tout $(\iota, \alpha)$ formé d'un intérieur prétopologique flou $\iota$ et d'une adhérence prétopologique floue $\alpha$ un opérateur $\psi: \mathcal{P}_{L}(X) \rightarrow \mathcal{P}_{L}(X)$ qui représenterait fidèlement la donnée de $(\iota, \alpha)$ : ne serait-ce que parmi celles qui vérifient

$$
\psi \vee I d=\alpha
$$

on ne peut exiger que pour tout $\epsilon \in \mathcal{P}_{L}(X)$, la donnée de $\psi(\epsilon)$ détermine celle de $\iota(\epsilon)$ et de $\alpha(\epsilon)$; il suffit pour s'en convaincre de considérer le cas où $L=\mathbf{I}, X=\{*\}$, $\epsilon=\frac{1}{2}$, et $\alpha(\epsilon)=1$, puisqu'alors nécessairement $\psi(\epsilon)=1$, quel que soit $\iota(\epsilon)$.

Prendre acte de cette difficulté ne doit nous conduire à renoncer ni à l'aspect « dialectique », qu'illustrent en particulier les définitions de Gentilhomme, de Dubois \& Prade ou d'Adnadjevič, ni à la considération des frontières en tant que lieux, mais simplement à ne plus exiger de ces dernières qu'elles représentent fidèlement la structure dialectique. Les définitions suivantes découlent de ce constat, en séparant l'idée d'espace dialectique de celle d'espace muni en outre d'un opérateur de frontières floues, pour la définition duquel nous ferons appel à l'idée intuitive selon laquelle une frontière mesure en quelque sorte l'écart qui sépare l'intérieur de l'adhérence, mesure pour laquelle il s'avère possible de se ramener à une opération définie dans $L$, donc indépendante de l'ensemble sous-jacent $X$. On disposera ainsi à la fois d'un moyen de produire diverses frontières, retrouvant ainsi la plupart des frontières définies antérieurement, et de les classer ${ }^{59}$.

DÉFINITION 31 (Espaces dialectiques flous). Un espace $L$-dialectique (ou espace dialectique flou) est un ensemble $X$ muni d'un couple de prétopologies $(\iota, \alpha) \in$ $\mathcal{F} \mathcal{P} i(X) \times \mathcal{F P} a(X)$. Un morphisme d'espaces dialectiques flous (ou application continue) $f:\left(X_{1}, \iota_{1}, \alpha_{1}\right) \rightarrow\left(X_{2}, \iota_{2}, \alpha_{2}\right)$ est une application $X_{1} \rightarrow X_{2}$ à la fois conti-

\footnotetext{
${ }^{58} \mathrm{Cf}$. la section 9 .

${ }^{59}$ Nous renvoyons à l'appendice (sections 5.4.12. et 5.4.13.) pour une présentation catégorielle de ces espaces, dont découle immédiatement la possibilité de procéder à toutes les constructions usuelles (produits cartésiens ou fibrés, sommes amalgamées, structures induites, etc.).
} 
nue vis-à-vis des prétopologies intérieures $\iota_{1}$ et $\iota_{2}$ et des prétopologies d'adhérence $\alpha_{1}$ et $\alpha_{2}$. On définit de même les espaces dialectiques flous de divers types.

Étant donné un treillis $L$, nous noterons $L^{\diamond}$ l'ensemble $\left\{(i, a) \in L^{2}, i \leq a\right\}$ muni de la relation d'ordre définie pour tout $\left(\left(i_{1}, a_{1}\right),\left(i_{2}, a_{2}\right)\right) \in\left(L^{\diamond}\right)^{2}$ par

$$
\left(i_{1}, a_{1}\right) \preceq\left(i_{2}, a_{2}\right) \Leftrightarrow\left\{\begin{array}{l}
i_{1} \geq i_{2} \\
a_{1} \leq a_{2}
\end{array}\right.
$$

DÉFINITION 32 (Séparateurs). Un séparateur sur le treillis L est une application croissante $\delta: L^{\diamond} \rightarrow L$ telle que $\delta(0,1)=1$ et $\delta(0,0)=\delta(1,1)=0$. Un séparateur $\delta$ sera dit :

- intègre, s'il vérifie $\forall a \in L, \delta(a, a)=0$,

- efficace, s'il vérifie $\forall(i, a) \in L^{\diamond}, i<a \Rightarrow \delta(i, a)>0$,

- modéré, s'il vérifie $\forall(i, a) \in L^{\diamond}, \delta(i, a) \leq a$,

- normal, s'il est à la fois intègre, efficace et modéré.

Munis de l'ordre induit par celui du treillis $L^{L^{\diamond}}$,

$$
\delta_{1} \leq \delta_{2} \Leftrightarrow \forall(i, a) \in L^{\diamond}, \delta_{1}(i, a) \leq \delta_{2}(i, a),
$$

l'ensemble $S_{L}$ des séparateurs constitue un treillis complet, de même que l'ensemble de séparateurs intègres et celui des modérés.

DÉFINITION 33 (Espaces à frontières floues). Un espace à frontières floues est un espace dialectique $(X, \iota, \alpha)$ muni d'un couple

$$
(t, \delta) \in \mathbf{2} \times S_{L}
$$

Si $\left(t_{1}, \delta_{1}\right) \leq\left(t_{2}, \delta_{2}\right)$, les morphismes de $\left(X_{1}, t_{1}, \delta_{1}, \iota_{1}, \alpha_{1}\right)$ dans $\left(X_{2}, t_{2}, \delta_{2}, \iota_{2}, \alpha_{2}\right)$ sont les morphismes dialectiques $\left(X_{1}, \iota_{1}, \alpha_{1}\right) \rightarrow\left(X_{2}, \iota_{2}, \alpha_{2}\right)$. Si $\left(t_{1}, \delta_{1}\right) \not{L}\left(t_{2}, \delta_{2}\right)$, il n'y a pas de morphisme du premier espace à frontières floues vers le second. L'ordre d'un espace à frontières floues $(X, t, \delta, \iota, \alpha)$ est $t$, son séparateur est $\delta$. Nous dirons aussi que $(X, t, \delta, \iota, \alpha)$ est un espace à frontières floues de type $(t, \delta)$. Si ı est une prétopologie floue de type $\mathcal{W}_{1} \in\{\mathcal{V}, \mathcal{D}, \mathcal{I}, \mathcal{T}\}$ et $\alpha$ de type $\mathcal{W}_{2}$, nous dirons encore que $(X, t, \delta, \iota, \alpha)$ est un espace à frontières floues de type $\left(t, \delta, \mathcal{W}_{1}, \mathcal{W}_{2}\right)$. Etant donné $(X, t, \delta, \iota, \alpha)$ un espace à frontières floues, la frontière floue associée est l'opérateur $\psi: \mathcal{P}_{L}(X) \rightarrow \mathcal{P}_{L}(X)$ défini par

$$
\psi=\alpha^{t}(\delta(\iota, \alpha))
$$

ò̀ $\alpha^{0}=I d$ et $\alpha^{1}=\alpha$.

DÉFInition 34. Soit $\psi: \mathcal{P}_{L}(X) \rightarrow \mathcal{P}_{L}(X)$ tel que $\psi(0)=\psi(1)=0$. On dit que $\psi$ est une frontière floue sur $X$ s'il existe une structure à frontières floues $(t, \delta, \iota, \alpha)$ sur $X$ telle que $\psi$ soit la frontière floue associée à cette structure. 
Une frontière floue $\psi$ sur $X$ sera dite modérée s'il existe une structure à frontière floue $(t, \delta, \iota, \alpha)$ dont la frontière associée soit $\psi$ et telle que $\delta$ soit un séparateur modéré. On définit de façon analogue le fait pour une frontière floue d'être intègre, efficace ou normale.

Exemples. On définit les séparateurs suivants $L^{\diamond} \rightarrow L$ :

$-\delta_{0}(i, a)=\neg i \wedge a$,

$-\delta_{W}(i, a)=\rho \neg i \wedge a$,

$-\delta_{P}(i, a)=\left\{\begin{array}{l}a \text { si } a>i, \\ 0 \text { sinon. }\end{array}\right.$

Les deux premiers sont efficaces et modérés mais non intègres, le troisième est normal. On en déduit, concernant les frontières dialectiques suivantes qui généralisent celles de même nom données antérieurement essentiellement dans le contexte de la topologie floue, les propriétés suivantes :

- la frontière $\psi_{0}=\delta_{0}(\iota, \alpha)$, généralisation dialectique de la frontière floue naïve définie par la formule (15), est efficace et modérée d'ordre 0 ,

- la frontière dialectique de Warren, $\psi_{W}=\alpha \delta_{W}(\iota, \alpha)$, est efficace et modérée d'ordre 1,

- la frontière dialectique de $\mathrm{Pu}$ Si-li, $\psi_{P}=\alpha \delta_{P}(\iota, \alpha)$, est normale d'ordre 1,

- la frontière dialectique de Wu Guang-qing et Zheng Chong-you, $\psi_{W Z}=\delta_{P}(\iota, \alpha)$, est normale d'ordre 0 .

On notera que l'ensemble des structures à frontières floues sur $X$ auxquelles une frontière floue donnée $\psi$ se trouve associée n'est généralement pas un treillis, de sorte que, par exemple, rien n'interdit a priori qu'une frontière intègre, efficace et modérée ne soit pas normale, simplement parce qu'elle posséderait ces propriétés en vertu de structures à frontières floues distinctes.

\section{CONCLUSION}

Les transformations sur des ensembles flous sont adaptées en analyse d'image quand les régions ne peuvent pas être définies de manière nette et précise. Le jeu de Go, par sa nature visuelle, est dans ce cas.

Bruno Bouzy [1995]

Nous espérons que les considérations développées dans cet article contribueront notamment à attirer l'attention des chercheurs en sciences humaines sur les possibilités de formalisations de modes non fusionnels de relations dont la dialectique se trouve bien exprimée par la nature à la fois liante et séparatrice des frontières. D'un point de vue mathématique, cet article ne constitue toutefois qu'une étape préliminaire dans l'étude, notamment catégorielle, du concept de frontière. Ainsi, n'avons-nous pas considéré ici le problème de la formalisation de l'idée de frontière 
entre deux ou plusieurs régions d'un espace, non plus que celle des frontières comme lieux en quelque sorte antérieurs à ce dont elles seraient les frontières.

Même en ce qui concerne la seule notion de frontière d'une région d'un espace, à laquelle nous nous sommes limité dans cet article, nous n'en sommes encore qu'au début de notre étude. Ainsi, nous n'avons pas abordé ici la question de la formalisation des frontières en relation avec l'idée très intéressante de connexité floue. Plus généralement, la carte des catégories en jeu et des foncteurs utiles quant aux concept de frontière reste largement à établir, en particulier dans le cas flou.

Diverses autres pistes semblent intéressantes à considérer, telle que l'étude des relations entre algorithmique et formalisation - dans quelle mesure l'élaboration d'algorithmes de reconnaissance automatique de frontières utilise-t-elle et enrichitelle la formalisation mathématique des frontières? - ou encore la prise en compte à un niveau fondamental de la fractalité des frontières, comme nous y invite l'exemple de morphisme connectif considéré à la fin de la section 2.2.

\section{APPENDICES}

\subsection{LES ESPACES DE RIESZ}

La contribution de F. Riesz à l'élaboration de la topologie générale a été étudiée par Thron [1997, p. 21-29]. Précisons ici la portée, en terme de séparation topologique, des axiomes considérés par le mathématicien hongrois [Riesz, 1907, 1909] pour les points d'accumulation. On vérifie que le deuxième axiome - un point d'accumulation pour une partie l'est encore pour une partie plus vaste - et le troisième - un point d'accumulation pour l'union de deux parties l'est encore pour au moins l'une des deux - sont équivalents à la définition habituelle des espaces topologiques. Le premier axiome -vis-à-vis d'une partie finie, tous les points de l'espace sont isolés - est une condition de séparation qui revient à exiger que les singletons soient fermés.

Le quatrième axiome - une partie admettant un point d'accumulation, il existe une partie de cette partie admettant encore ce point comme point d'accumulation mais vis-à-vis de laquelle tout autre point donné par avance sera isolé - est plus délicat à analyser. Montrons sur un exemple ${ }^{60}$ qu'il conduit à considérer des espaces qui ne sont pas nécessairement séparés.

Sur l'ensemble $X=\mathbf{N}^{2} \cup\{A, B\}$ obtenu en ajoutant deux points au carré cartésien de l'ensemble $\mathbf{N}$ des entiers naturels, on définit une adhérence $\alpha$ de la façon suivante : à toute partie $E$ de $X$, on ajoute le point $A$ si $E$ admet une colonne comportant un nombre infini d'éléments, et par ailleurs on y ajoute le point $B$ si le nombre de colonnes non vides de $E$ est infini. On vérifie immédiatement qu'une telle adhérence est topologique, c'est-à-dire qu'elle est idempotente $\left(\alpha^{2}=\alpha\right)$ et que l'adhérence de l'union de deux parties est l'union des adhérences. Toute partie de $X$ qui contient $\{A, B\}$ étant fermée, la topologie définie par cette adhérence est discrète sur $\mathbf{N}^{2}$, et les seuls points susceptibles d'être des points d'accumulation sont $A$ et $B$.

\footnotetext{
${ }^{60}$ Cet exemple a été construit à partir d'une remarque d'un utilisateur du forum fr.sci.math, Hubert Quatreville.
} 
On vérifie alors très facilement que le quatrième axiome de Riesz est satisfait pour cet espace, tandis que $A$ et $B$ ne peuvent être séparés par des ouverts disjoints.

Par contre, la réciproque est évidemment vérifiée : tout espace topologique séparé est un espace de Riesz. Les espaces de Riesz constituent donc bien une classe intermédiaire entre celle des espaces de Hausdorff et celle des espaces de Fréchet.

\subsection{UN EXEMPLE SUR $\mathbf{R}$ DE TOPOLOGIE ADMETTANT LES INTERVALLES POUR CONNEXES}

Notons $\mathcal{R}$ la topologie usuelle de $\mathbf{R}$, et soit $\mathcal{T}$ la topologie sur $\mathbf{R}$ engendrée par $\mathcal{R} \cup\{\mathbf{Q}\}$. La topologie $\mathcal{T}$ contient nécessairement toute partie de $\mathbf{R}$ de la forme $U \cup(V \cap \mathbf{Q})$, où $(U, V) \in \mathcal{R}^{2}$ et, réciproquement, l'ensemble de ces parties constitue une topologie contenant $\mathcal{R}$ et $\{\mathbf{Q}\}$, donc contenant $\mathcal{T}$.

Montrons que les parties de $\mathbf{R}$ qui sont connexes pour la topologie $\mathcal{T}$ sont exactement les intervalles. Puisque $\mathcal{T}$ est plus fine que $\mathcal{R}$, les parties non connexes pour celle-ci continuent à ne pas l'être pour celles-là. Pour la réciproque, commençons par montrer que $\mathbf{R}$ est $\mathcal{T}$-connexe. Soit, pour $i \in\{1,2\}$, deux $\mathcal{T}$-ouverts $W_{i}=U_{i} \cup\left(V_{i} \cap \mathbf{Q}\right)$ tels que $W_{1} \cup W_{2}=\mathbf{R}$ et $W_{1} \cap W_{2}=\emptyset$. On a

$$
\left\{\begin{array}{l}
U_{1} \cap U_{2}=\emptyset \\
U_{1} \cap V_{2} \cap \mathbf{Q}=\emptyset \\
V_{1} \cap U_{2} \cap \mathbf{Q}=\emptyset \\
V_{1} \cap V_{2} \cap \mathbf{Q}=\emptyset
\end{array}\right.
$$

La densité, pour la topologie usuelle, de $\mathbf{Q}$ dans $\mathbf{R}$ entraîne alors

$$
\left\{\begin{array}{l}
U_{1} \cap U_{2}=\emptyset \\
U_{1} \cap V_{2}=\emptyset \\
V_{1} \cap U_{2}=\emptyset \\
V_{1} \cap V_{2}=\emptyset
\end{array}\right.
$$

soit, en posant, $Z_{i}=U_{i} \cup V_{i}$

$$
Z_{1} \cap Z_{2}=\emptyset
$$

Mais $Z_{i} \supset W_{i}$, d'où

$$
Z_{1} \cup Z_{2}=\mathbf{R}
$$

et la $\mathcal{R}$-connexité de $\mathbf{R}$ entraîne alors sa $\mathcal{T}$-connexité.

Soit maintenant $I$ une partie $\mathcal{R}$-connexe quelconque, c'est-à-dire un intervalle. Si $I$ est réduit à un point, il est connexe pour toute topologie. Sinon, $I \cap \mathbf{Q}$ est dense dans $I$ pour la topologie induite sur $I$ par la topologie usuelle, et le raisonnement mené précédemment pour $\mathbf{R}$ s'applique encore à $I$.

\subsection{CATÉGORIES À TREILlis DE STRUCTURES (GÉNÉRALITÉS)}

Je dois à Jean Bénabou d'avoir attiré mon attention sur ce que j'appelle les catégories à treillis de structures, qui constituent un cas particulier (et particulièrement simple) 
de catégories fibrées [Grothendieck, 1960 ; Bénabou, 1983]. L'intérêt de considérer cette classe de catégories tient notamment au fait que toutes limites et colimites ${ }^{61}$ qui existent dans la catégorie de base (par exemple les ensembles) existent également dans toute catégorie à treillis de structures construite sur cette base.

\subsubsection{Définition des catégories à treillis de structures}

On note $\mathbf{T}$ la catégorie dont les objets sont les treillis complets et dont les flèches sont les applications entre treillis qui préservent les inf 62 .

DÉFInition 35. Soit $\mathbf{X}$ une catégorie et $T: \mathbf{X}^{o p} \rightarrow \mathbf{T}$ un foncteur (contravariant) qui, à tout objet $X$ de $\mathbf{X}$, associe un treillis complet $T_{X}$ et, à tout morphisme $f$ : $X \rightarrow Y$, associe un morphisme de treillis $T_{f}: T_{Y} \rightarrow T_{X}$. La catégorie à treillis de structures de base $\mathbf{X}$ associée à $T$ est la catégorie notée $\mathbf{X}_{T}$ dont les objets sont les couples de la forme $(X, \mathcal{X})$, où $X$ est un objet de base et $\mathcal{X}$ un élément du treillis $T_{X}$, et dont les morphismes $f:(X, \mathcal{X}) \rightarrow(Y, \mathcal{Y})$ sont les $\mathbf{X}$-morphismes $f: X \rightarrow Y$ tels que, dans le treillis $T_{X}$, soit vérifiée la relation $\mathcal{X} \leq T_{f}(\mathcal{Y})$.

Nous dirons que $T$ est le foncteur (contravariant) structurant de la catégorie $\mathbf{X}_{T}$. Si $\mathcal{X}_{1}$ et $\mathcal{X}_{2}$ sont deux éléments de $T_{X}$ tels que $\mathcal{X}_{1} \leq \mathcal{X}_{2}$, nous dirons que $\mathcal{X}_{1}$ est plus fin ou plus discret que $\mathcal{X}_{2}$, ce dernier étant dit plus épais, plus grossier ou plus indiscret que le premier. Pour tout $\mathbf{X}$-morphisme $f$, l'application $T_{f}$ sera désignée comme l'application de transfert de structures associée à $f$, et sera le plus souvent notée simplement $f^{*}$, ou parfois $f^{\#}$.

On obtient une définition équivalente en faisant appel aux foncteurs covariants $S: \mathbf{X} \rightarrow \mathbf{S}$, où $\mathbf{S}$ désigne la catégorie des treillis complets avec pour morphismes les applications entre treillis qui préservent les sup. Notant $f_{!}$la flèche de $\mathbf{S}$ associée à $f$ par $S$, la catégorie à treillis de structures de base $\mathbf{X}$ associée à $S$, notée $\mathbf{X}_{S}$, a pour objets les couples $(X, \mathcal{X})$ avec $X$ objet de base et $\mathcal{X}$ élément du treillis $S_{X}$, et pour morphismes $f:(X, \mathcal{X}) \rightarrow(Y, \mathcal{Y})$ les morphismes $f: X \rightarrow Y$ telles que, dans le treillis $S_{Y}$, soit vérifiée la relation $f_{!}(\mathcal{X}) \leq \mathcal{Y}$.

Étant donné un foncteur $T: \mathbf{X}^{o p} \rightarrow \mathbf{T}$, le foncteur covariant $S$ qui conduit à la même catégorie à treillis de structures que $T$ est défini sur les objets $X$ de $\mathbf{X}$ par $S_{X}=T_{X}$, et sur les morphismes $f: X \rightarrow Y$ par

$$
\forall \mathcal{X} \in S_{X}, f_{!}(\mathcal{X})=\bigwedge\left\{\mathcal{Y} \in S_{Y}, \mathcal{X} \leq f^{*}(\mathcal{Y})\right\}
$$

Réciproquement, étant donné un foncteur covariant $S: \mathbf{X} \rightarrow \mathbf{S}$, le foncteur contravariant $T$ qui conduit à la même catégorie à treillis de structures que $S$ est défini sur les objets $X$ de $\mathbf{X}$ par $T_{X}=S_{X}$, et sur les morphismes $f: X \rightarrow Y$ par

$$
\forall \mathcal{Y} \in T_{Y}, f^{*}(\mathcal{Y})=\bigvee\left\{\mathcal{X} \in T_{X}, f_{!}(\mathcal{X}) \leq \mathcal{Y}\right\}
$$

\footnotetext{
${ }^{61}$ Pour toutes les notions usuelles en théorie des catégories, la référence classique reste [Mac Lane, 1971].

${ }^{62}$ Ces applications sont nécessairement croissantes et préservent les 1 des treillis.
} 
Les treillis $S_{X}$ et $S_{Y}$ étant considérés comme des catégories, on vérifie que $f_{!}$est adjoint à gauche ${ }^{63}$ du foncteur $f^{*}$ : on a en effet, pour tout $(\mathcal{X}, \mathcal{Y}) \in S_{X} \times S_{Y}$

$$
f_{!}(\mathcal{X}) \leq \mathcal{Y} \Leftrightarrow \mathcal{X} \leq f^{*}(\mathcal{Y})
$$

Remarquons que la définition des catégories à treillis de structures sur une catégorie de base $\mathbf{X}$ peut s'interpréter comme celle d'un foncteur $\mathbf{S}^{\mathbf{X}} \rightarrow \mathbf{C a t}$ qui, à chaque foncteur covariant $S: \mathbf{X} \rightarrow \mathbf{S}$ associe la catégorie $\mathbf{X}_{S}$ et, naturellement, à chaque transformation naturelle un foncteur.

Signalons enfin que, la catégorie de base étant la catégorie des ensembles, si le foncteur structurant associe à tout ensemble réduit à un point un treillis de structures lui-même réduit à une seule structure, alors la catégorie définie par ce foncteur constitue un cas particulier de ce que l'on appelle une « catégorie topologique ${ }^{64}$. Parmi les exemples de catégories à treillis de structures donnés plus loin (section 5.4.), celles des graphes, des relations d'équivalence, des espaces connectifs intègres, des espaces prétopologiques de divers types et en particulier des espaces topologiques, des espaces dialectiques sont des catégories topologiques. Par contre, outre la catégorie des prédicats et celle des espaces connectifs (non nécessairement intègres), la catégorie des ensembles flous et celles des divers types d'espaces dialectiques ou prétopologiques flous ne sont pas des catégories topologiques ${ }^{65}$.

Dans la suite et sauf mention contraire, $T$ désigne un foncteur structurant contravariant sur la catégorie de base $\mathbf{X}$, et $S$ le foncteur structurant covariant associé.

\subsubsection{Structures initiales}

Soit $X$ un objet de $\mathbf{X},\left(Y_{i}, \mathcal{Y}_{i}\right)_{i \in I}$ une famille d'objets de $\mathbf{X}_{T}$ et $\left(f_{i}: X \rightarrow Y_{i}\right)_{i \in I}$ une famille de $\mathbf{X}$-morphismes de même index $I$. Les structures $\mathcal{X} \in T_{X}$ telles que, pour tout $i \in I, f_{i}$ soit un $\mathbf{X}_{T}$-morphisme de $(X, \mathcal{X})$ dans $\left(Y_{i}, \mathcal{Y}_{i}\right)$ sont caractérisées par

$$
\mathcal{X} \leq \bigwedge_{i \in I} f_{i}^{*} \mathcal{Y}_{i}
$$

Ainsi, $\bigwedge_{i \in I} f_{i}^{*} \mathcal{Y}_{i}$ est la moins fine des structures sur $X$ qui fassent de tous les $f_{i}$ des $\mathbf{X}_{T}$-morphismes : c'est la structure initiale sur $X$ pour la famille des $f_{i}$ et des $\mathcal{Y}_{i}$, notée aussi $\left[f_{i}\right]^{*}\left[\mathcal{Y}_{i}\right]$.

\subsubsection{Structures finales}

Soit $Y$ un objet de $\mathbf{X},\left(X_{i}, \mathcal{X}_{i}\right)_{i \in I}$ une famille d'objets de $\mathbf{X}_{T}$ et $\left(f_{i}: X_{i} \rightarrow Y\right)_{i \in I}$ une famille de $\mathbf{X}$-morphismes de même index $I$. Les structures $\mathcal{Y} \in T_{Y}$ telles que, pour

\footnotetext{
${ }^{63}$ C'est la raison pour laquelle nous avons préféré noter $f$ ! le foncteur de transport final des structures plutôt que $f_{*}$, souvent utilisé pour désigner plutôt un adjoint à droite d'un foncteur $f^{*}$, comme c'est par exemple le cas pour le foncteur image directe des faisceaux sur un espace topologique.

${ }^{64}$ Sur les catégories topologiques, cf. par exemple Herrlich [1983].

${ }^{65}$ En particulier, la catégorie des espaces topologiques flous au sens de Chang n'est pas une catégorie topologique, contrairement à la variante proposée dans sa thèse par R. Lowen [1974, $1976]$.
} 
tout $i \in I, f_{i}$ soit un $\mathbf{X}_{T}$-morphisme de $\left(X_{i}, \mathcal{X}_{i}\right)$ dans $(Y, \mathcal{Y})$ sont caractérisées par

$$
\bigvee_{i \in I}\left(f_{i}\right)_{!}\left(\mathcal{X}_{i}\right) \leq \mathcal{Y}
$$

Ainsi, $\bigvee_{i \in I}\left(f_{i}\right)_{!}\left(\mathcal{X}_{i}\right)$ est la plus fine des structures sur $Y$ qui fassent de tous les $f_{i}$ des $\mathbf{X}_{T}$-morphismes : c'est la structure finale sur $Y$ pour la famille des $f_{i}$ et des $\mathcal{X}_{i}$, notée aussi $\left[f_{i}\right] !\left[\mathcal{X}_{i}\right]$.

\subsubsection{Monomorphismes, épimorphismes}

On vérifie facilement qu'un $\mathbf{X}_{T}$-morphisme est un monomorphisme (resp. épimorphisme) si et seulement si le $\mathbf{X}$-morphisme sous-jacent est lui-même un monomorphisme (resp. épimorphisme).

\subsubsection{Structures induites}

Étant donné un objet $(X, \mathcal{X})$ de $\mathbf{X}_{T}$, et $j: A \hookrightarrow X$ un $\mathbf{X}$-monomorphisme représentant un $\mathbf{X}$-sous-objet de $X$, la structure induite par $\mathcal{X}$ sur $A$ est la structure initiale $j^{*}(\mathcal{X})$.

\subsubsection{Quotient par une relation d'équivalence}

Dans le cas où $\mathbf{X}$ est la catégorie des ensembles $\mathbf{S e t s}$, une relation d'équivalence $\mathcal{R}$ sur un ensemble $X$ est déterminée par la surjection canonique $s: X \rightarrow X / \mathcal{R}$ qui lui est associée. Etant donnée une structure $\mathcal{X} \in T_{X}$, le quotient de $(X, \mathcal{X})$ par $\mathcal{R}$ est alors défini par

$$
(X, \mathcal{X}) / \mathcal{R}=\left(s(X), s_{!}(\mathcal{X})\right)
$$

\subsubsection{Théorème de transfert}

Étant donnée une catégorie à treillis de structures $\mathbf{X}_{S}$, notons $U: \mathbf{X}_{S} \rightarrow \mathbf{X}$ le foncteur d'oubli qui à tout espace $(X, \mathcal{X})$ associe l'objet de base sous-jacent $X$ et à toute flèche de $\mathbf{X}_{S}$ elle-même en tant que flèche de $\mathbf{X}$. Notons encore $O: \mathbf{X} \rightarrow \mathbf{X}_{S}$ le foncteur discret qui conserve les flèches et qui à tout objet $X$ de $\mathbf{X}$ associe

$$
O(X)=\left(X, 0_{S_{X}}\right)
$$

et $I: \mathbf{X} \rightarrow \mathbf{X}_{S}$ le foncteur indiscret qui conserve les flèches et qui à tout objet $X$ de $\mathbf{X}$ associe

$$
I(X)=\left(X, 1_{S_{X}}\right)
$$

On vérifie facilement la double adjonction

$$
O \dashv U \dashv I
$$

de sorte que $U$ préserve nécessairement les limites et les colimites. Le théorème suivant complète ce résultat en assurant le transfert des limites (resp. colimites) dans le sens $\mathbf{X} \rightarrow \mathbf{X}_{S}$. 
THÉORÈME 17 (Transfert). Soit $\mathbf{X}_{S}$ une catégorie à treillis de structures, $\mathbf{J}$ une petite catégorie et $\widetilde{A}=\left(A_{j}, \mathcal{A}_{j}\right)$ un diagramme dans $\mathbf{X}_{S}$ de type $\mathbf{J}$. On note $A=\left(A_{j}\right)$ le diagramme sous-jacent dans $\mathbf{X}$.

Si A admet une limite projective de morphismes projectifs une famille $\left(f_{j}\right)_{j \in \mathbf{J}}$, alors $\widetilde{A}$ admet également une limite projective

$$
\lim _{\leftarrow \mathbf{J}}(\widetilde{A})=\left(\lim _{\leftarrow \mathbf{J}}(A),\left[f_{j}\right]^{*}\left[\mathcal{A}_{j}\right]\right),
$$

avec les mêmes morphismes projectifs.

Si $A$ admet une limite inductive de morphismes inductifs une famille $\left(g_{j}\right)_{j \in \mathbf{J}}$, alors $\widetilde{A}$ admet également une limite inductive

$$
\lim _{\rightarrow \mathbf{J}}(\widetilde{A})=\left(\lim _{\rightarrow \mathbf{J}}(A),\left[g_{j}\right] !\left[\mathcal{A}_{j}\right]\right),
$$

avec les mêmes morphismes inductifs.

DÉMONSTRATION. La démonstration consiste à vérifier que les conditions contenues dans la définition d'une limite (resp. colimite) sont ici satisfaites ${ }^{66}$.

\subsubsection{Limites et colimites classiques}

Un objet initial d'une catégorie peut-être vu comme limite inductive du diagramme vide, de sorte que si $\mathbf{X}$ admet un objet initial $0_{\mathbf{X}}$, alors $\left(0_{\mathbf{X}}, 0_{S\left(0_{\mathbf{x}}\right)}\right)$ est objet initial de $\mathbf{X}_{S}$.

Un objet final d'une catégorie peut-être vu comme limite projective du diagramme vide, de sorte que si $\mathbf{X}$ admet un objet final $1_{\mathbf{X}}$, alors $\left(1_{\mathbf{X}}, 1_{S\left(1_{\mathbf{x}}\right)}\right)$ est objet final de $\mathbf{X}_{S}$.

S'il existe dans $\mathbf{X}$, le produit cartésien dans $\mathbf{X}_{S}$ est obtenu en appliquant le théorème de transfert avec $\mathbf{J}=\{\bullet, \bullet\}$. Par exemple pour $\mathbf{X}=$ Sets, étant donnés deux ensembles structurés $\widetilde{X}_{1}=\left(X_{1}, \mathcal{X}_{1}\right)$ et $\widetilde{X}_{2}=\left(X_{2}, \mathcal{X}_{2}\right)$ et $p_{i}: X_{1} \times X_{2} \rightarrow X_{i}$ la $i^{\text {ème }}$ projection $(i \in\{1,2\})$, on aura, avec les mêmes projections

$$
\tilde{X}_{1} \times \tilde{X}_{2}=\left(X_{1} \times X_{2}, p_{1}^{*} \mathcal{X}_{1} \wedge p_{2}^{*} \mathcal{X}_{2}\right)
$$

On obtient de la même façon les produits fibrés $(\mathbf{J}=\{\bullet \rightarrow \bullet \leftarrow \bullet\}$, les noyaux $(\mathbf{J}=\{\bullet \rightrightarrows \bullet\})$ et, par dualité, les sommes amalgamées, les conoyaux, etc.

\subsubsection{Produits de foncteurs structurants}

$\mathbf{S}$ désignant toujours la catégorie des treillis complets avec pour morphismes les applications qui préservent les sup, on sait ${ }^{67}$ que la catégorie fonctorielle $\mathbf{S}^{\mathbf{X}}$, dont les objets sont les foncteurs structurants (covariants) $\mathbf{X} \rightarrow \mathbf{S}$ et dont les morphismes

\footnotetext{
${ }^{66}$ Pour une démonstration détaillée, cf. par exemple [Dugowson, 2003, p. 34].

${ }^{67}$ Cf. par exemple Mac Lane [1971, p. 112].
} 
sont les transformations naturelles entre ces foncteurs, admet, quelle que soit la catégorie de base $\mathbf{X}$ utilisée, toutes les limites ou colimites qui existent dans $\mathbf{S}$, ces (co)limites étant déterminées ponctuellement. Or, le produit cartésien de deux treillis complets existe toujours dans $\mathbf{S}$ - c'est le treillis obtenu en munissant le produit cartésien des ensembles sous-jacents de la relation d'ordre définie par la conjonction des deux relations d'ordre considérées - de sorte qu'il est toujours possible de faire le produit cartésien de deux foncteurs structurants, ou plus généralement d'un nombre fini de tels foncteurs. Un tel produit cartésien de foncteurs structurants s'interprète à son tour comme foncteur structurant d'une catégorie dont les objets seront caractérisés par la donnée d'une famille finie de structures sur les objets de base, les morphismes devant respecter ces différentes structures.

\subsection{CATÉGORIES À TREILlis De STRUCTURES (EXEMPles)}

Les sections suivantes présentent succinctement les principales catégories d'ensemble à treillis de structures - donc de la forme $\mathbf{S e t s}_{T}$ - qui ont implicitement été définies au cours du présent article. Comme nous l'avons noté dans notre conclusion, cette inscription du cas ensembliste dans la formulation générale des catégories à treillis de structures, au-delà du cas particulier où $\mathbf{X}=$ Sets, devrait être comprise comme une incitation à l'étude de structures analogues définies sur des catégories de base plus générales.

\subsubsection{Prédicats}

La catégorie des prédicats est $\operatorname{Sets}_{\mathcal{P}}$ où $\mathcal{P}$ désigne le foncteur structurant contravariant de Sets dans $\mathbf{T}$ défini par :

- pour tout ensemble $X, \mathcal{P}_{X}=\mathcal{P}(X)$ est le treillis des parties de $X$,

- pour toute application $f: X \rightarrow Y, \mathcal{P}_{f}=f^{*}$ est définie par

$$
\forall B \in \mathcal{P}(Y), f^{*}(B)=f^{-1}(B)
$$

\subsubsection{Graphes}

Pour tout graphe simple et non orienté ${ }^{68}(G, \mathcal{A})$, appelons structure graphique l'ensemble $\mathcal{A}$. L'ensemble de telles structures sur un ensemble $G$ donné constitue un treillis complet pour l'inclusion. La catégorie des graphes est alors $\mathbf{G}=\mathbf{S e t s}_{\mathcal{G}}$ où $\mathcal{G}$ désigne le foncteur structurant contravariant de Sets dans T défini par

- pour tout ensemble $X, \mathcal{G}_{X}$ est le treillis des structures graphiques sur $X$,

- pour toute application $f: X \rightarrow Y, \mathcal{G}_{f}=f^{*}$ est définie par

$$
\forall \mathcal{B} \in \mathcal{G}_{Y}, f^{*}(\mathcal{B})=\left\{\left\{x_{1}, x_{2}\right\} \in \mathcal{P}(X), f\left(\left\{x_{1}, x_{2}\right\}\right) \in \mathcal{B}\right\}
$$

\footnotetext{
${ }^{68}$ Nous utilisons ici la définition 1 donnée p. 93.
} 


\subsubsection{Relations d'équivalence}

Les relations d'équivalence sur un ensemble $X$ peuvent être vues comme des graphes particuliers ${ }^{69}$, une telle relation d'équivalence étant plus fine qu'une autre si le graphe de la première est inclus dans celui de la seconde.

Étant donnée une relation d'équivalence $\mathcal{R}$ sur $X$, notons $x_{1} \equiv_{\mathcal{R}} x_{2}$ le fait que $x_{1}$ et $x_{2}$ soient équivalents pour $\mathcal{R}$. La catégorie des relations d'équivalence est alors $\operatorname{Sets}_{\mathcal{E}}$ où $\mathcal{E}$ désigne le foncteur structurant contravariant de Sets dans $\mathbf{T}$ défini par ;

- pour tout ensemble $X, \mathcal{E}_{X}$ est le treillis des relations d'équivalence sur $X$,

- pour toute application $f: X \rightarrow Y, \mathcal{E}_{f}=f^{*}$ est définie par

$$
\forall \mathcal{R} \in \mathcal{E}_{Y}, \forall\left(x_{1}, x_{2}\right) \in X^{2}, x_{1} \equiv_{f^{*}(\mathcal{R})} x_{2} \Leftrightarrow f\left(x_{1}\right) \equiv_{\mathcal{R}} f\left(x_{2}\right) .
$$

\subsubsection{Espaces connectifs}

La catégorie des espaces connectifs a pour objets les espaces connectifs et pour morphismes les applications connectives (cf. supra, p. 95, la définition 3). La catégorie des espaces connectifs intègres, ici notée $\mathbf{K I}$, en est la sous-catégorie pleine dont les objets sont les espaces connectifs intègres. L'ensemble des structures connectives intègres sur un ensemble donné constitue un treillis complet pour l'inclusion [Dugowson, 2003, p.9], aussi KI cö̈ncide-t-elle avec la catégorie structurée par le foncteur contravariant $\mathcal{K} \mathcal{I}:$ Sets $^{o p} \rightarrow \mathbf{T}$ qui, à tout ensemble, associe le treillis complet des structures connectives intègres qui peuvent être définies sur cet ensemble et qui, à toute application $f: X \rightarrow Y$, associe l'application de transfert de structures $\mathcal{K} \mathcal{I}(f)=f^{*}$ définie pour toute structure connective intègre $\mathcal{L}$ sur $Y$ par

$$
f^{*}(\mathcal{L})=\{K \in \mathcal{P}(X), f(K) \in \mathcal{L}\} .
$$

Comme toute catégorie d'ensembles à treillis de structures, ces deux catégories admettent toutes limites et colimites. À la question près (et sans grand intérêt) de savoir si la partie vide doit ou non être considérée comme connexe, KI coïncide avec la catégorie Zus (pour Zusammenhangsräume) introduite par Börger [1983] sous le nom de « category of connectivity spaces and connectivity morphisms ». Börger montre que cette catégorie est une catégorie topologique ${ }^{70}$, mais qu'elle n'est pas cartésienne close $^{71}$. On vérifie toutefois [Dugowson, 2003, 2007] que KI est une catégorie monoïdale fermée ${ }^{72}$.

\subsubsection{Espaces prétopologiques}

On vérifie [Dugowson, 2003, p.60] que l'ensemble $\mathcal{P} \mathcal{R}_{X}$ des adhérences prétopologiques sur un ensemble $X$ donné est, pour la relation d'ordre définie par $\alpha_{1} \leq \alpha_{2} \Leftrightarrow$

\footnotetext{
${ }^{69}$ Elles peuvent aussi être vues comme des espaces topologiques particuliers.

${ }^{70}$ Cf. supra, p. 136.

${ }^{71}$ C'est-à-dire que le produit cartésien par un espace donné n'admet pas, en général, d'adjoint à droite. Sur la notion de catégorie cartésienne close (ou fermée), voir par exemple Mac Lane [1971].

${ }^{72}$ Autrement dit qu'il existe sur KI un produit tensoriel et une structure connective sur l'ensemble des morphismes connectifs d'un espace connectif vers un autre avec une certaine relation d'adjonction entre ces données; sur la notion de catégorie monoïdale fermée, cf. par exemple Mac Lane [1971].
} 
$\alpha_{1} \subset \alpha_{2}$, un treillis complet de plus petit élément $0_{\mathcal{P R}_{X}}$ l'adhérence discrète (qui à toute partie associe elle-même) et de plus grand élément $1_{\mathcal{P} \mathcal{R}_{X}}$ l'adhérence grossière ou indiscrète (qui à toute partie non vide associe $X$ ).

La catégorie $\mathbf{P R}$ des espaces prétopologiques est une catégorie d'ensembles à treillis de structures, avec pour catégorie de base $\mathbf{X}$ la catégorie $\mathbf{S e t s}$ des ensembles, et pour foncteur structurant covariant de $\mathbf{X}$ dans $\mathbf{S}$ le foncteur $\mathcal{P} \mathcal{R}$ défini par :

- pour tout ensemble $X, \mathcal{P} \mathcal{R}_{X}$ est le treillis complet des adhérences sur $X$,

- pour toute application $f: X \rightarrow Y, \mathcal{P R}_{f}=f_{\text {! }}$ est définie par

$$
\forall \alpha \in \mathcal{P} \mathcal{R}_{X}, \forall B \in \mathcal{P}(Y), f_{!}(\alpha)(B)=\left(\bigcup_{A \in \mathcal{P}(X), f(A)=B} f(\alpha(A))\right) \cup B .
$$

La version contravariante fait appel à $f^{\#}$, défini par

$$
\forall \beta \in \mathcal{P} \mathcal{R}_{Y}, \forall A \in \mathcal{P}(X), f^{\#}(\beta)(A)=f^{-1}(\beta(f(A))) .
$$

En ce qui concerne la catégorie $\mathbf{P V}$ des espaces prétopologiques de type $\mathcal{V}$, on a une formulation contravariante identique à la précédente [Dugowson, 2003, p. 64] mais avec un foncteur structurant $\mathcal{P} \mathcal{V}$ n'associant à chaque ensemble que les adhérences de type $\mathcal{V}$ sur cet ensemble. Par contre, la formulation covariante est plus simple; on vérifie en effet que, dans ce cas, $f$ ! est donné par

$$
\forall \alpha \in \mathcal{P} \mathcal{V}_{X}, \forall B \in \mathcal{P}(Y), f_{!}(\alpha)(B)=f\left(\alpha\left(f^{-1}(B)\right)\right)
$$

Remarque. Une variante des formulations précédentes consiste à s'appuyer sur une représentation des structures prétopologiques non plus par les adhérences, mais par les intérieurs, ce qui suppose, pour que les formulations obtenues soient équivalentes, d'orienter les intérieurs également du discret vers le grossier, de sorte que pour deux opérateurs intérieurs $\iota_{1}$ et $\iota_{2}$ définis sur un ensemble $X$, on aura

$$
\iota_{1} \preceq \iota_{2} \quad \Leftrightarrow \quad \iota_{1} \supset \iota_{2}
$$

\subsubsection{Espaces topologiques}

La catégorie TOP des espaces topologiques peut être vue comme la catégorie d'ensembles à treillis de structures définie par le foncteur structurant contravariant $\mathcal{T O P}:$ Sets $^{o p} \rightarrow \mathbf{T}$ qui à tout ensemble associe le treillis complet des topologies (ensembles d'ouverts) sur cet ensemble (treillis pour lequel la relation d'ordre est opposée à ce que l'on appelle parfois la force - et plus justement la finesse d'une topologie, de sorte que le 0 de ce treillis est la topologie discrète, et son 1 la topologie grossière), et qui à toute application $f: X \rightarrow Y$ associe l'application de transfert de structure $f^{\#}: \mathcal{T} \mathcal{O} \mathcal{P}_{Y} \rightarrow \mathcal{T} \mathcal{O} \mathcal{P}_{X}$ définie pour toute topologie $\mathcal{T}$ sur $Y$ comme la moins fine des topologies sur $X$ qui rende $f$ continue, c'est-à-dire

$$
f^{\#}(\mathcal{T})=\left\{f^{-1}(V), V \in \mathcal{T}\right\}
$$


Ce n'est là, à vrai dire, que l'une des nombreuses formulations possibles de la catégorie des espaces topologiques comme catégorie à treillis de structures, puisqu'on peut aussi l'exprimer en terme de fermés, d'adhérences, d'intérieurs ou de frontières, et pour chaque cas dans la version covariante ou dans la version contravariante [Dugowson, 2003, p. 26-28 et 75-83].

\subsubsection{Espaces dialectiques}

La définition des espaces dialectiques et des morphismes dialectiques que nous avons posée section 2.5.1. résulte certes des considérations développées au début de ladite section, mais surtout du fait qu'elles-mêmes conduisent à la considération d'une catégorie à treillis de structure naturellement définie en terme de produit de foncteurs ${ }^{73}$. En effet, la catégorie $\mathbf{D}$ des espaces dialectiques dont les objets et les morphismes sont donc ceux de la définition 10, s'interprète comme étant $\mathbf{S e t s}_{\mathcal{P R}^{2}}$, catégorie des ensembles structurés par le foncteur $\mathcal{D}=\mathcal{P} \mathcal{R}^{2}$, carré cartésien du foncteur structurant des espaces prétopologiques, ce qui signifie simplement qu'un espace dialectique est un ensemble muni d'un couple de structures prétopologiques et que les morphismes sont les applications respectant les structures respectivement en jeu dans la définition de ces espaces.

On remarque qu'il existe plusieurs variantes dans cette définition, selon que l'on fasse appel uniquement aux adhérences prétopologiques, ou bien également aux intérieurs prétopologiques, ou encore aux frontières dialectiques. Pour les intérieurs définis sur un ensemble $X$, l'ordre $\preceq$ retenu pour le treillis qu'ils constituent est celui défini en section 5.4.5. par la formule (30). Pour les frontières, l'ordre retenu est induit simplement par l'inclusion : l'opérateur frontière discret donne des frontières toujours vides tandis que l'opérateur frontière grossier donne, pour toute partie propre, la frontière la plus épaisse à savoir l'espace entier.

Notons par ailleurs que la catégorie DV des espaces dialectiques de type $\mathcal{V}$, c'est-à-dire des ensembles munis d'un couple de prétopologies de type $V$, s'interprète également comme catégorie à treillis de structure, à savoir $\operatorname{Sets}_{\mathcal{P V}^{2}}$.

\subsubsection{Quelques foncteurs entre les catégories précédentes}

Nous avons vu en section 2.3. qu'il n'y avait pas de façon unique ni probablement « optimale »d'associer un opérateur frontière à tout espace connectif intègre. Du moins en existe-t-il d'assez naturelles, en l'occurrence fonctorielles, à commencer par celles que l'on obtient en composant par un foncteur quelconque ${ }^{74}$ de $\mathbf{P R}$ dans $\mathbf{D}$ le foncteur $\mathrm{S}: \mathbf{K I} \rightarrow \mathbf{P R}$ ainsi défini [Dugowson, 2003, p. 90] :

- pour tout espace connectif intègre $(X, \mathcal{K})$, on pose $\mathrm{S}(X, \mathcal{K})=(X, \alpha)$ avec

$$
\forall A \in \mathcal{P}(X), \alpha(A)=\{x \in X, \exists V \in \mathcal{P}(A) \backslash\{\emptyset\}, V \cup\{x\} \in \mathcal{K}\},
$$

- pour toute application connective $f:(X, \mathcal{K}) \rightarrow(Y, \mathcal{L})$, on prend $\mathrm{S}(f)=f$.

\footnotetext{
${ }^{73}$ Cf. la section 5.3.9.

${ }^{74}$ Pour une présentation succincte de quelques uns de ces foncteurs, cf. Dugowson [2005].
} 
Dans le cas où on se limite aux espaces dialectiques de type $\mathcal{V}$, l'association considérée aux sections 2.3. et 2.5.2. qui à toute frontière dialectique $\varphi$ fait correspondre la structure connective $\mathcal{L}_{\varphi}$ constituée de ce que nous avons appelé les parties connexes aux frontières (cf. la formule 9) s'interprète, lorsque de plus on associe elle-même à toute application continue, comme un foncteur L : DV $\rightarrow \mathbf{K I}$. Ainsi, un espace dialectique de type $\mathcal{V}$ étant donné par son opérateur frontière $\varphi$, on a

$$
\mathrm{L}(X, \varphi)=\left(X, \mathcal{L}_{\varphi}\right) .
$$

Par composition avec le foncteur diagonal de $\mathbf{P V}$ dans $\mathbf{D V}$ qui à tout espace prétopologique de type $\mathcal{V}$ associe l'espace dialectique obtenu en prenant deux fois l'adhérence considérée, on obtient un foncteur également noté L, de $\mathbf{P V}$ dans KI. On définit de même un foncteur L sur TOP, par restriction.

L'association définie section 2.5.2. qui à tout espace dialectique fait correspondre l'espace connectif déterminé par ses parties connexes s'interprète, lorsque de plus on associe elle-même à toute application continue, comme un foncteur $\mathrm{K}: \mathbf{D} \rightarrow \mathbf{K I}$. Ainsi, un espace dialectique étant donné par son opérateur frontière $\varphi$, on a

$$
\mathrm{K}(X, \varphi)=\left(X, \mathcal{K}_{\varphi}\right) .
$$

Par composition avec le foncteur diagonal de $\mathbf{P R}$ dans $\mathbf{D}$ qui à tout espace prétopologique associe l'espace dialectique obtenu en prenant deux fois l'adhérence considérée, on obtient le foncteur de $\mathbf{P R}$ dans $\mathbf{K I}$, également noté $\mathrm{K}$, qui se trouve implicitement défini en section 2.4. par la proposition 6. L'association définie section 2.2. qui à tout espace topologique fait correspondre l'espace connectif déterminé par ses parties connexes s'interprète à son tour comme la restriction à TOP du foncteur $K$ précédent, restriction que nous pourrons noter également $K$ et dont il faut noter qu'il est, d'après la proposition 4 , distinct du foncteur L : TOP $\rightarrow \mathbf{K I}$.

Dans les sections suivantes, $L$ désigne un treillis flou ${ }^{75}$.

\subsubsection{Ensembles flous}

De la première note de bas de page par laquelle Zadeh précise la définition des ensembles flous que nous avons rappelée en section 3.1., il découle une possible hésitation quant à la catégorie susceptible de représenter au mieux les objets introduits par Zadeh, selon qu'on les considère plutôt comme des ensembles flous ou des sousensembles flous. En un sens, c'est cette seconde option qui sera retenue par Goguen. Curieusement, bien qu'une partie conséquente de son article de 1967 - dans lequel il définit les ensembles $L$-flous - soit consacrée aux catégories (en particulier sur la façon de fuzzyfier les catégories par les morphismes) ce n'est que dans son article de 1974 que Goguen pose la définition de la catégorie des $L$-ensembles, $\operatorname{Sets}(L)$, pour tout ensemble ordonné $(L, \leq)$ :

DÉFinition 36 (Catégorie des $L$-ensembles). Les objets de $\operatorname{Sets}(L)$ sont les $L$ ensembles. Un morphisme $\alpha \rightarrow \beta$ de L-ensembles $\alpha: X \rightarrow L$ et $\beta: Y \rightarrow L$ est une application $f: X \rightarrow Y$ telle que, pour tout $x \in X, \alpha(x) \leq \beta(f(x))$.

\footnotetext{
${ }^{75}$ Cf. supra la définition 15.
} 
Dans le cas où $L$ est un treillis complet, la catégorie de $\operatorname{Goguen} \operatorname{Sets}(L)$ coïncide avec la catégorie à treillis de structures $\operatorname{Sets}_{\mathcal{P}_{L}}$, où $\mathcal{P}_{L}$ désigne le foncteur structurant sur Sets qui à tout ensemble $X$ associe le treillis complet $\mathcal{P}_{L}(X)=\left(L^{X}, \leq\right) \in \mathbf{T}$ des applications de $X$ dans $L$ et qui, dans sa version contravariante, associe à toute application ensembliste $f: X \rightarrow Y$ le morphisme entre treillis $f^{*}: \mathcal{P}_{L}(Y) \rightarrow \mathcal{P}_{L}(X)$ défini pour tout $\gamma: Y \rightarrow L$ par

$$
f^{*}(\gamma)=\gamma \circ f
$$

$f^{*}(\gamma)$ représente alors l'image réciproque du sous-ensemble flou $\gamma$ de $Y$, tandis que la version covariante de la formulation de cette catégorie comme catégorie à treillis de structure nous donne l'expression de l'image directe par $f$ d'un sous-ensemble flou $\epsilon$ de $X$-image directe qu'avec les notations générales que nous avons indiquées nous devrions écrire $f_{!}(\epsilon)$ mais que nous avons convenu dans la section 3.1. de noter dans le cas présent simplement $f(\epsilon)$ - à savoir l'expression donnée par la formule (13).

Cette catégorie $\operatorname{Sets}_{\mathcal{P}_{L}}$ vient parmi les toutes premières auxquelles on peut penser lorsqu'on veut donner des exemples abstraits de catégories à treillis de structures, $L^{X}$ représentant sans doute l'expression la plus simple que l'on puisse imaginer pour associer un treillis complet (non constant) à chaque ensemble $X$. Mis à part les cas triviaux, les ensembles flous représentent donc la catégorie à treillis de structure la plus simple. Certes, l'idée d'associer à tout ensemble $X$ le treillis complet $\mathcal{P}(X)$ de ses parties, et à toute application $f: X \rightarrow Y$ le morphisme entre treillis $f^{*}: \mathcal{P}(Y) \rightarrow \mathcal{P}(X)$ défini pour tout $\Gamma \in \mathcal{P}(Y)$ par

$$
f^{*}(\Gamma)=f^{-1}(\Gamma)
$$

paraît plus simple encore : associé à ce choix de $\mathcal{P}$ comme foncteur structurant, on obtient alors comme catégorie à treillis de structures la catégorie des prédicats que nous avons considérée comme premier exemple (section 5.4.1.), et dont chaque objet est en quelque sorte défini par la manifestation au sein d'un contexte $X$ d'une partie $P \subset X$. Cependant, si l'on représente les parties des ensembles par leurs fonctions caractéristiques, l'équation (32) apparaît comme un cas particulier de (31), et la catégorie des prédicats comme un cas particulier de la catégorie $\operatorname{Sets}(L)$, lorsque $L=\mathbf{2}$.

Davantage qu'une généralisation de la catégorie des ensembles $\operatorname{Sets}_{\tilde{\mathbf{1}}}=\operatorname{Sets}(\mathbf{1})$, les ensembles flous apparaissent donc plutôt comme une généralisation de la catégorie des prédicats d'ensembles, $\operatorname{Sets}_{\mathcal{P}}=\operatorname{Sets}_{\tilde{\mathbf{2}}}=\operatorname{Sets}(\mathbf{2})$, ce que nous avons exprimé plus haut en parlant de catégorie de sous-ensembles flous plutôt que d'ensembles flous.

Il en résulte par exemple que, dans la catégorie $\operatorname{Sets}(L)$, des sous-objets distincts d'un même objet peuvent représenter intuitivement le même sous-ensemble flou. Soit par exemple $X$ un ensemble et $1_{X}: X \rightarrow L$ le $L$-ensemble associé (autrement dit $1_{X}$ est la fonction constante sur $X$ de valeur $1_{L}=1$ ) ; l'injection canonique $\gamma_{1}: \emptyset \rightarrow L$ et la fonction constante nulle $\gamma_{2}: X \rightarrow L$ définissent alors deux sous-objets distincts de $1_{X}$, bien qu'on puisse aussi considérer qu'ils représentent tous deux le même 
sous-ensemble (vide) de $X$. On retrouve ainsi l'ensemble $\mathcal{P}_{L}(X)=L^{X}$ des sousensembles flous de $X$ en effectuant le quotient de l'ensemble des sous-objets de $1_{X}$ par la relation d'équivalence ainsi définie : un tel sous-objet $g$ pouvant être représenté de façon unique par un mono $i: \gamma \rightarrow 1_{X}$ tel que l'injection sous-jacente $i: \operatorname{dom}(\gamma) \hookrightarrow X$ soit canonique, on note $\chi_{g}$ l'ensemble flou de domaine $X$ tel que $\chi_{g}(x)=\gamma(x)$ si $x \in \operatorname{supp}(\gamma)$ et $\chi_{g}(x)=0$ si $x \notin \operatorname{supp}(\gamma)$; deux tels sous-objets $g_{1}$ et $g_{2}$ sont alors équivalents si $\chi_{g_{1}}=\chi_{g_{2}}$. Plus généralement, on retrouve ainsi l'ensemble des sous-ensembles flous d'un ensemble flou donné comme quotient par cette relation d'équivalence de l'ensemble des sous-objets de l'ensemble flou considéré.

\subsubsection{Espaces prétopologiques flous}

La catégorie FP des espaces prétopologiques flous a pour objets les espaces prétopologiques flous et pour morphismes les applications continues entre eux (voir la définition (30) en section 3.5.). On définit de même diverses sous-catégories pleines de FP correspondant aux différents types de prétopologies floues, dont la catégorie des espaces topologiques flous (voir plus loin la section 5.4.11.). Ces diverses catégories sont des catégories à treillis de structures. Ainsi, on a $\mathbf{F P}=\mathbf{S e t s}_{\mathcal{F} \boldsymbol{P} a}$, où $\mathcal{F P} a$ est le foncteur contravariant qui à tout ensemble $X$ associe le treillis complet $\mathcal{F P} a(X)$ des adhérences prétopologiques floues sur $X$, et qui à toute application $f: X \rightarrow Y$ associe l'application de transfert de structures $f^{\#}: \mathcal{F P} a(Y) \rightarrow \mathcal{F P} a(X)$ caractérisée par le fait qu'à toute adhérence floue $\beta$ sur $Y$ elle associe l'adhérence floue $f^{\#}(\beta)$ la moins fine sur $X$ qui rende continue $f$. Autrement dit, par définition de la continuité ici en jeu, $f^{\#}(\beta)$ est la moins fine des adhérences floues $\alpha$ sur $X$ telles que, pour toute partie floue $\epsilon \in \mathcal{P}_{L}(X)$, on ait

$$
f(\alpha(\epsilon)) \leq \beta(f(\epsilon)),
$$

autrement dit encore, en appliquant la formule (28) exprimant l'adjonction entre images directes et réciproques au couple formé de l'image directe $f=f_{\text {! }}$ et de l'image réciproque $f^{*}$ d'un sous-ensemble flou par $f$ (cf. les formules (13) et (31)), il s'agit de la moins fine des $\alpha$ telles que

$$
\forall \epsilon \in \mathcal{P}_{L}(X), \alpha(\epsilon) \leq f^{*}(\beta(f(\epsilon)))
$$

d'où finalement

$$
\forall \epsilon \in \mathcal{P}_{L}(X), f^{\#}(\beta)(\epsilon)=f^{*}(\beta(f(\epsilon))),
$$

formule qui généralise la formule (29).

On obtient une formulation équivalente en utilisant le treillis complet des intérieurs prétopologiques flous $\mathcal{F} \mathcal{P} i(X)$ à la place du treillis $\mathcal{F} \mathcal{P} a(X)$. Comme dans le cas net ainsi généralisé ${ }^{76}$, rappelons ${ }^{77}$ que l'ordre $\preceq$ du treillis $\mathcal{F} \mathcal{P} i(X)$ est opposé à celui qui découle de l'inclusion floue.

Par ailleurs, les catégories des espaces prétopologiques flous respectivement de type $\mathcal{V}, \mathcal{V}_{\mathcal{I}}, \mathcal{V}_{\mathcal{D}}$ et enfin des espaces topologiques flous s'écriront respectivement

\footnotetext{
${ }^{76} \mathrm{Cf}$. la formule (30) section 5.4.5.

${ }^{77} \mathrm{Cf}$. la section 3.5 .
} 
$\operatorname{Sets}_{\mathcal{F} \mathcal{P V}}, \operatorname{Sets}_{\mathcal{F} \mathcal{P V} \mathcal{I}}, \operatorname{Sets}_{\mathcal{F} \mathcal{P V D}}$ et $\mathbf{F T}=\operatorname{Sets}_{\mathcal{F} \mathcal{T}}$, pour les foncteurs structurants adéquats.

\subsubsection{Espaces topologiques flous}

La catégorie FT des espaces topologiques flous est la sous-catégorie pleine de FP constituée des espaces qui sont à la fois de type ${ }^{78} \mathcal{V}_{\mathcal{I}}$ et $\mathcal{V}_{\mathcal{D}}$. Il s'agit d'une catégorie à treillis de structures, de foncteur structurant le foncteur $\mathcal{F} \mathcal{T}$ obtenu à partir du foncteur $\mathcal{F P} a$ en se restreignant aux adhérences floues qui sont topologiques.

Une définition équivalente de la catégorie FT consiste à considérer une structure topologique floue sur un ensemble comme constituée de l'ensemble des parties floues invariantes pour une adhérence topologique floue donnée, parties qualifiées de fermées, ou encore des complémentaires floues de ces parties, qualifiées d'ouvertes. On retrouve de la sorte la définition 16.

\subsubsection{Espaces dialectiques flous}

La catégorie FD des espaces $L$-dialectiques (ou dialectiques flous) a pour objets et morphismes ceux donnés par la définition 31 (section 3.6.). Elle s'interprète comme la catégorie à treillis de structures $\operatorname{Sets}_{\mathcal{F} \mathcal{D}}$, avec $\mathcal{F} \mathcal{D}=\mathcal{F} \mathcal{P} i \times \mathcal{F} \mathcal{P} a$. Les catégories d'espaces dialectiques flous de divers types s'interprètent de façon analogue.

\subsubsection{Espaces à frontières floues}

La catégorie FB des espaces à frontières floues a pour objets et morphismes ceux donnés par la définition 33 (section 3.6.). Elle s'interprète comme la catégorie à treillis de structures $\operatorname{Sets}_{\mathcal{F} \mathcal{B}}$, avec $\mathcal{F B}=\overline{\mathbf{2}} \times \overline{S_{L}} \times \mathcal{F} \mathcal{P} i \times \mathcal{F} \mathcal{P} a$, où $\overline{\mathbf{2}}$ désigne le foncteur constant Sets $\rightarrow \mathbf{T}$ qui à tout ensemble associe le treillis $\mathbf{2}$ et $\overline{S_{L}}$ le foncteur constant qui à tout ensemble associe le treillis $S_{L}$ des séparateurs sur $L$. Les catégories d'espaces à frontières floues de divers types s'interprètent de façon analogue.

\subsection{ClASSIFICATION ET DÉNOMINATIONS DES ESPACES PRÉTOPOlOGIQUES}

Sur le modèle des espaces bitopologiques [Kelly, 1962], ont été introduits récemment des espaces bitopologiques flous [Chandrasekhara Rao, 2000, Das et Das, 2001]. Sampath Kumar [2000] étudie la connexité dans de tels espaces. Cet auteur introduit par ailleurs [2001] la notion d'espace suprabitopologique flou, construite à partir de la version floue [Abd El-Monsef et Ramadan, 1987] des espaces supratopologiques [Allam, Khedr, Mahmoud et Mashhour, 1983]. D'après Kent et Min [2002], les espaces supratopologiques ne sont rien d'autres que les espaces de clôture, c'est-à-dire ce que nous appelons les espaces prétopologiques de type $\mathcal{V}_{\mathcal{I}}$, tandis que dans la terminologie de Kent et Won Keun Min, les pretopological spaces, dont ils font remonter l'origine à Choquet [1948], désignent ce que Čech [1966] appelle des espaces de clôture et Belmandt [1993] (et nous avec) appelle les espaces prétopologiques de type $\mathcal{V}_{\mathcal{D}}$

\footnotetext{
${ }^{78}$ Les types de prétopologies floues sont donnés dans la définition 29.
} 


\section{NOTATIONS}

On indique ci-dessous la section (ou éventuellement les sections) où les notations sont introduites.

Ensembles

$\begin{array}{ll}\operatorname{card}(X) & \text { Cardinal de l'ensemble } X, 2.1 . \\ \mathbf{I} & \text { Intervalle réel }[0,1], \text { muni de la relation d'ordre usuelle. } \\ \mathbf{Q} & \text { Ensemble des rationnels, } 2.2 . \\ \mathbf{R} & \text { Espace des nombres réels, } 2.2 . \\ \mathbf{C} & \text { Corps des nombres complexes, } 2.2 .\end{array}$

Parties floues et opérateurs

$\neg$
$\neg A$
$\neg \epsilon$
$\kappa$
$\rho$
$\tilde{\varphi}=\varphi\urcorner$
$\lambda^{c}$
$\lambda \eta$
$\lambda \vee \eta$
$\lambda \cup \eta$
$\lambda \wedge \eta$
$\lambda \cap \eta$
$\lambda \leq \eta$
$\lambda \subset \eta$
$d o m$
$\mathcal{F} \mathcal{F}_{L}(X), \mathcal{F} \mathcal{F}(X)$
$I d$
$\mathcal{P}_{L}(X)$
$\mathcal{P}(X)$
$\mathcal{P} *(X)$
supp

Espaces, structures
Opérateur décroissant involutif dans un treillis, 3.2.

Complémentaire dans un ensemble d'une partie $A$ de cet ensemble, 1.1.

Complémentaire flou d'une partie floue $\epsilon$ d'un ensemble, 3.2.

Cour d'un ensemble flou, 3.4.5.

Adhérence de rehaussement, 3.4.3.

Frontière adjointe de la frontière $\varphi, 2.5 .1$.

$=\neg \lambda \neg, 1.1 ., 3.4 .3$.

$=\lambda \circ \eta$, composée de deux applications de $\mathcal{P}_{L}(X), 1.2$., 3.4.3.

$\epsilon \mapsto \lambda(\epsilon) \vee \eta(\epsilon), 3.4 .3$

$=\lambda \vee \eta$ dans le cas net, 1.2 .

$\epsilon \mapsto \lambda(\epsilon) \wedge \eta(\epsilon), 3.4 .3$.

$=\lambda \wedge \eta$ dans le cas net, 1.2 .

$\Leftrightarrow \forall \epsilon, \lambda(\epsilon) \leq \eta(\epsilon), 3.1$.

$\lambda \leq \eta$ dans le cas net, 1.2 .

Domaine d'un ensemble flou, 3.1 .

Treillis des ensembles à double-feuillets flous sur un ensemble $X, 3.4 .5$.

Application identique sur $\mathcal{P}_{L}(X), 1.2 ., 3.4 .3$.

$=L^{X}$, ensemble des parties $L$-floues d'un ensemble $X, 3.1 ., 5.4 .9$.

$=\mathcal{P}_{\mathbf{2}}(X)$, ensemble des parties de l'ensemble $X, 1.1$., 1.2., 5.4.1.

$=\mathcal{P}(X) \backslash\{\emptyset, X\}$, ensemble des parties propres de $X, 1.2$.

Support d'un ensemble flou, 3.1.

Ensemble des parties manifestement connexes, 2.4., 2.5.2.

Ensemble des structures dialectiques nettes sur $X, 2.5 .1$.

Transfert inverse de structure par $f, 5.3 .1$.

Transfert direct de structure par $f, 5.3 .1$.

Ensemble des adhérences prétopologiques floues sur $X, 3.5$.

Ensemble des intérieurs prétopologiques flous sur $X, 3.5$.

Structure connective (usuelle) associée à une frontière, 2.3., 2.4., 2.5.2.

Structure des connexes aux frontières associée à $\varphi, 2.3 ., 2.5 .2$.

Ensemble des séparateurs de $L, 3.6$.

Types de prétopologies, 2.4.

Espaces prétopologiques définis par adhérence, 2.4.

Espace dialectique net définis par deux adhérences, 2.5.1.

Espace dialectique net défini par frontière dialectique, 2.5.1.

Espaces connectifs, 2.2. 
Frontières

$\tilde{\varphi}=\varphi\urcorner$
$\mu$
$\nu$
$\Phi_{\mathcal{K}}$
$\Phi_{\mathcal{P}}(X)$
$\Phi_{\mathcal{V}}(X)$
$\Phi_{\mathcal{D}}(X)$
$\Phi_{\mathcal{I}}(X)$
$\Phi_{\mathcal{T}}(X)$
$\Phi_{X}$
$\psi_{0}$
$\psi_{1}$
$\Psi_{0}$
$\psi_{P}$
$\psi_{W}$
$\psi_{W Z}$
$\psi_{Y}$

Catégories

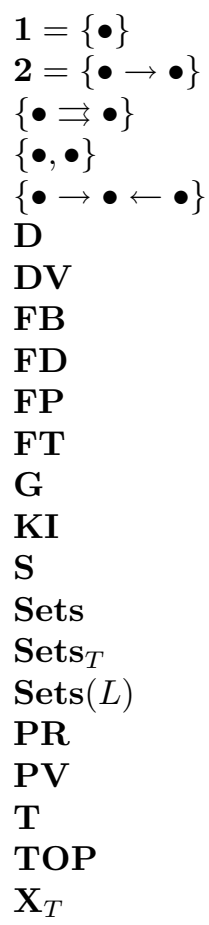

frontière adjointe de la frontière $\varphi, 2.5 .1$.

Frontière chinoise, 2.1.

Frontière niponne, 2.1.

Ensemble des $\mathcal{K}$-frontières, 2.3.

Ensemble des frontières prétopologiques sur $X, 2.4$.

Ensemble des frontières prétopologiques de type $\mathcal{V}$ sur $X, 2.4$.

Ensemble des frontières prétopologiques de type $\mathcal{V}_{\mathcal{D}}$ sur $X, 2.4$.

Ensemble des frontières prétopologiques de type $\mathcal{V}_{\mathcal{I}}$ sur $X, 2.4$.

Ensemble des frontières topologiques sur $X, 2.4$.

Ensemble des frontières dialectiques nettes sur $X, 2.5 .1$.

frontière floue naïve (ou de $\mathrm{Liu} \& \mathrm{Pu}$ ), 3.4.1., 3.4.2., 3.6.

frontière associée à $\Psi_{0}, 3.4 .5$.

Frontière de Dubois et Prade, 3.4.5.

Frontière de $\mathrm{PuSi}$-li, 3.4.4., 3.6.

Frontière de Warren, 3.4.3., 3.6.

Frontière de Wu Guang-qing et Zheng Chong-you, 3.4.8., 3.6.

Frontière de Ying Mingsheng, 3.4.9.

5.4.9.

3.2., 5.4.9.

5.3 .8 .

5.3 .8 .

5.3.8.

$=\operatorname{Sets}_{\mathcal{P R}^{2}}$, catégories des espaces dialectiques nets, 5.4.7.

$=\operatorname{Sets}_{\mathcal{P} \mathcal{V}^{2}}$, catégorie des espaces dialectiques de type $\mathcal{V}$, 5.4.7.

$=\operatorname{Sets}_{\mathcal{F B}}$, catégorie des espaces à frontières floues, 5.4.13.

$=\operatorname{Sets}_{\mathcal{F} \mathcal{D}}$, catégorie des espaces dialectiques flous, 5.4.12.

$=\operatorname{Sets}_{\mathcal{F P} a} \simeq \operatorname{Sets}_{\mathcal{F P} i}$, catégorie des espaces prétopologiques flous, 5.4.10.

$=\operatorname{Sets}_{\mathcal{F} \mathcal{T}}$, catégorie des espaces topologiques flous, 5.4.11.

Catégorie des graphes simples non orientés, 5.4.2.

Catégorie des espaces connectifs intègres, 5.4.4.

Catégorie des treillis complets (morphismes préservant les sup), 5.3.1.

Catégorie des ensembles, 5.3.6.

Catégorie des ensembles structurés par le foncteur $T, 5.4$.

$=\operatorname{Sets}_{\mathcal{P}_{L}}$, catégorie des ensembles $L$-flous, 5.4.9.

$=\operatorname{Sets}_{\mathcal{P R}}$, catégories des espaces prétopologiques nets 5.4.5.

Catégorie des espaces prétopologiques de type $\mathcal{V}$, 5.4.5.

Catégorie des treillis complets (morphismes préservant les inf), 5.3.1.

Catégorie des espaces topologiques, 5.4.6.

Catégorie des objets de $\mathbf{X}$ structurés par le foncteur $T$, 5.3.1. 
Foncteurs structurants

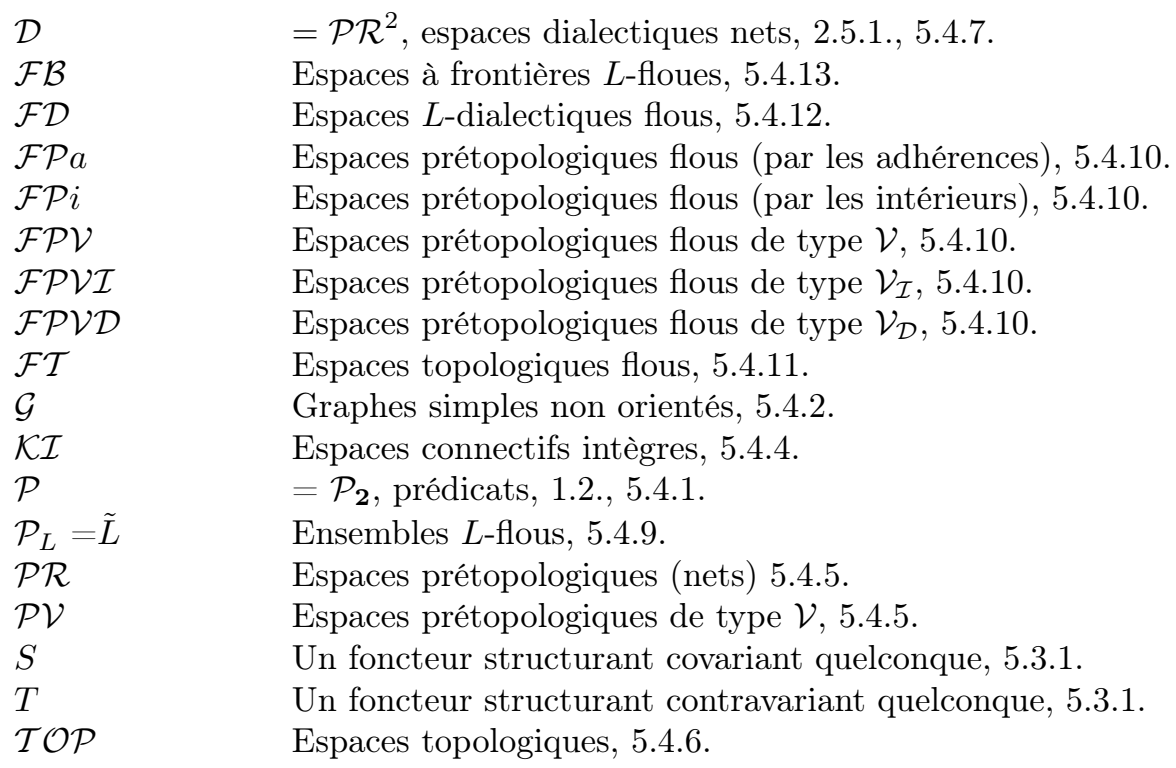

Autres foncteurs

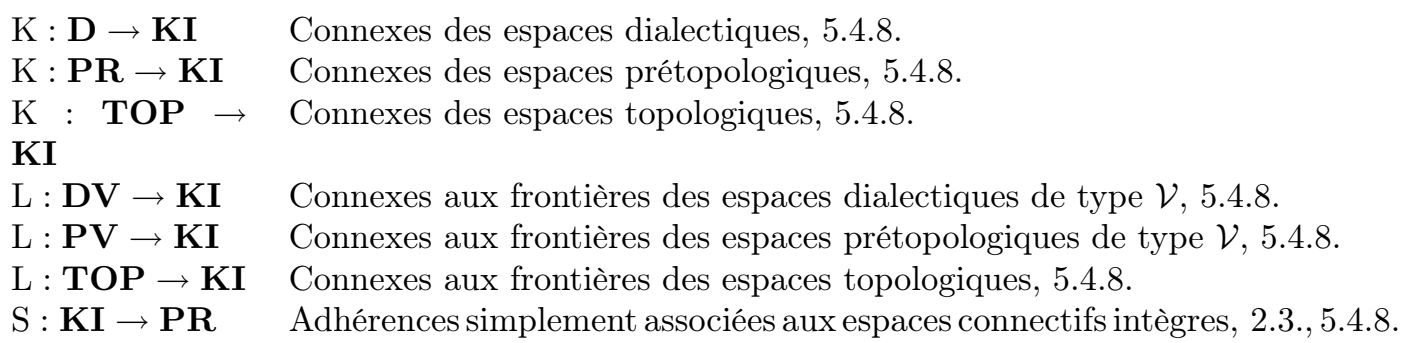

\section{BIBLIOGRAPHIE}

La date entre crochets indiquée devant chaque référence et qui est celle employée dans le corps du texte correspond autant que possible à la date de constitution initiale du document concerné - date de conférence, de signature ou de soumission d'un article - et peut donc être strictement antérieure à la date d'édition indiquée dans la référence.

ABD AL-MONSEF M.E., RAMAdAN A.E., [1987], "On fuzzy supratopological spaces", Indian J. Pure Appl. Math. 18, 1987, p. 322-329.

ADNADJEVIČ D., [1986], "Dimension of fuzzy spaces", Fuzzy sets and systems 26, 1988, p. 85-92. ALBERT G., [1941], "A note on quasi-metric spaces", Bulletin of the American Mathematical Society 47, 1941, p. 479-482.

ALlAM A.A., KHEDR F.H., MAHMOUd F.S., MASHHOUR A.S., [1983], "On supratopological spaces", Indian J. Pure Appl. Math. 14, 1983, p. 502-510.

ANDERSON M., [1997], "Les frontières : un débat contemporain", Contrôles : frontières, identités. Les enjeux autour de l'immigration et de l'asile, Cultures et Conflits 26-27, 1997. [Consultable en ligne à : http ://conflits.revues.org/article.php3 ?id_article=211]. 
BADARD R., [1981], "Fuzzy pretopological spaces and their representations", Journal of mathematical analysis and applications 81, 1981 p. 378-390.

BALANZAT M., [1951], "On the metrisation of quasi-metric spaces", Gaz. Mat. Lisboa 12[50], 1951, p. 91-94.

Belmandt Z., [1993], Manuel de prétopologie et ses applications, Paris, Hermès, 1993.

BÉNABOU J., [1983], "Fibered categories and the foundations of naive category theory", The Journal of Symbolic Logic 50[1], 1985, p.10-37.

BENNINGTON G., [1992], "La frontière infranchissable", Le passage des frontières (autour du travail de Jacques Derrida), Actes du colloque tenu à Cerisy du 11 au 21 juillet 1992, Paris, Galilée, 1994.

BÖRGER R., [1981], Kategorielle Beschreibungen von Zusammenhangsbegriffen, Thèse, Fernuniversität Hagen, 1981.

BÖRGER R., [1983], "Connectivity spaces and component categories", Categorical topology, International Conference on Categorical Topology, Berlin, Heldermann, 1984.

BOURBaki N., [1990], "Topologie générale", chapitres 1 à 4 des Eléments de Mathématique, Paris, Masson, 1990 [nouveau tirage].

BOUZY B., [1995], "Les ensembles flous au jeu de Go", Actes des Rencontres françaises sur la logique floue et ses applications, Paris, LFA-95,1995, p. 334-340

BRISSAUd M., [1975], "Les espaces prétopologiques", Comptes rendus de l'Académie des Sciences 280, A, p. 705-708.

BROWn L.M., [1986], "Fuzzy topology and bitopological spaces; Anouncement", Third National Meeting on Topology, Trieste, 9-12 juin 1986.

Camus A., [1951], L’homme révolté, Paris, Gallimard, 1951.

ČECH E., [1966], Topological spaces, New York, Interscience Publishers, 1966.

CHANDRASEKhara RAO K., [2000], "Fuzzy bitopological spaces", Bulletin of Pure and Applied Sciences, Section E, Mathematics and Statistics, 19[1], 2000, p. 139-146.

CHANG C.L., [1968], "Fuzzy topological spaces", Journal of mathematical analysis and applications 24, 1968 p. $182-190$.

Choquet G., [1948], "Convergences", Ann. Univ. Grenoble Sect. Sci. Math. Phys. 23, 1948, p. 57112 .

CUChILlo-IBÁÑEz E., TARRÉS J., [1997], "On the boundary of fuzzy sets", Fuzzy Sets and Systems 89, 1997, p. 113-119.

DAS N. R., DAS P., [2001], "Fuzzy bitopological space induced by fuzzy quasi-pseudo-norm and its conjugate", Fuzzy Sets and Systems 119[3], 2001, p. 539-542.

DUBOIS D., PRADE H., [1981], "Twofold fuzzy sets : an approach on the representation of sets with fuzzy boundaries based on possibility and necessity measures, Fuzzy mathematics 3[4], 1983, p. 5376 ; [partial version presented at the $3^{\text {rd }}$ International Seminar on Fuzzy Set Theory, Linz, Austria, 1981].

DUGOWson s., [2003], Espaces connectifs et espaces de partage, 118 pages, [non publié, copie sur demande à l'auteur].

DUGOwson S., [2005], « Les catégories et le passage des frontières 》, Colloque Impact des catégories, octobre 2005, École normale supérieure, Paris. [L'enregistrement vidéo de cette conférence est disponible en ligne : http ://www.diffusion.ens.fr/index.php ?res=conf\&idconf=894].

DUGOWson s., [2006(a)], « Pour le partage des frontières : connexité, généricité, fractalité », séminaire Pensée des Sciences de Charles Alunni, 24 mars 2006, École normale supérieure, Paris. [Un enregistrement vidéo de cette intervention peut être visionné à partir de la page :

http ://s.dugowson.free.fr/recherche].

Dugowson s., [2006(b)], « Les mathématiques des frontières floues », Pour La Science 350, décembre 2006. 
Dugowson s., [2007], «Attractions borroméennes », Séminaire Mamuphi (Mathématiques, Musique, Philosophie), dirigé par François Nicolas, Charles Alunni et Moreno Andreatta, 27 janvier 2007, École normale supérieure, Paris. [L'enregistrement vidéo de cette conférence est disponible en ligne : http ://www.diffusion.ens.fr/index.php ?res=conf\&idconf=1639].

FRÉCHet M., [1928], Les espaces abstraits, Paris, Gauthiers-Villars, 1928.

Gentilhomme y., [1968], "Les ensembles flous en linguistique", Cahiers de Linguistique Théorique et Appliquée 5, Bucarest, 1968, p. 47-63.

GRothendieck A., [1960], "Catégories fibrées et descente", Séminaire de géométrie algébrique du Bois-Marie 1960/1961 (SGA 1), exposé n ${ }^{\circ}$ VI, IHES, Paris, 1963. [Reprint : Lecture Notes in Mathematics 224, Berlin, Springer-Verlag, 1971].

GoGuen J.A., [1967], "L-Fuzzy Sets", Journal of mathematical analysis and applications 18, 1967, p. 145-174.

Goguen J.A., [1973], "Concept Representation in Natural and Artificial Languages : Axioms, Extensions and Applications for Fuzzy Sets", Int. J. Man-Machine Studies 6, 1974, p. 513-561.

GUenard F., LELIÈvre G., [1985], Compléments d'analyse, vol. 1 : Topologie, première partie, Cahiers de Fontenay, Fontenay-aux-Roses, E.N.S., 1985.

Hall E.T., [1959] , The Silent Language, Garden City (New York), Doubleday \& Company Inc., 1959. [Traduction française : Le langage silencieux, Paris, Seuil, 1984].

HERRLiCh H., [1983], "Categorical topology 1971-81", General Topology and its Relation to Analysis and Algebra IV, Berlin, Heldermaann Verlag, 1983.

HÖHle U., KLEMEnt E.P., Rodabaugh s.E. (eds), [1989], Applications of category theory to fuzzy subsets, Dordrecht, Kluwer Academic Publishers, 1992.

HUtTon B., [1980], "Products of fuzzy topological spaces", Topology Appl. 11, 1980, p. 59-67.

KAINZ W., TANG X., [2002], "Analysis of topological relations between fuzzy regions in a general fuzzy topological space", Joint ISPRS Commission IV Symposium, Spatial Data Handling and 95th Annual CIG Geomatics Conference, Ottawa, Canada.

KELlY J.C., [1962], " Bitopological spaces", Proc. London Math. Soc. 3, [13], 1963, p. 71-89.

KENT D.C., MIN W.K., [2002], "Neighborhood spaces", International Journal of Mathematics and Mathematical Sciences 32, 2002, 7, p. 387-399.

KURATOwski K., [1920], «Sur l'opération A de l'analysis situs », Fundamenta Mathematicae 3, 1922, p. 182-199.

KURATOWSKI K., [1955], Wstęp do teorii mnogości i topologii, Warszawa, PWN, 1955.

KURATOWski K., [1961] Introduction to set theory and topology, Oxford, Pergamon Press Ltd, Reading, Addison-Wesley, 1962.

KURATOWSKI K., [1966], Introduction à la théorie des ensembles et à la topologie, Genève, L'enseignement mathématique, 1966.

LACAN J., [1973], « Ronds de ficelle », Séminaire du 22 octobre 1973, Encore (Séminaire, livre XX), texte établi par J.-A. Miller, Paris, Seuil, 1975.

LACAN J., [1975], Le sinthome, Le séminaire livre XXIII, 1975-1976, texte établi par J.-A. Miller, Paris, Seuil, 2005.

LECLERC J., [2006], « Les frontières linguistiques », La vitalité des petites langues, Québec, TLFQ, Université Laval, 16 janv. 2006 :

http ://www.tlfq.ulaval.ca/AXL/Langues/2vital_petiteslangues.htm

LIU Y.-M., PU P.-M., [1976], "Fuzzy Topology (I). Neighborhood structure of a fuzzy point and Moore-Smith convergence", Sichuan Daxue Xuebao 1, 1977, p. 31-50 [en chinois].

LIU Y.-M., PU P.-M., [1980], "Fuzzy Topology (I). Neighborhood structure of a fuzzy point and Moore-Smith convergence", Jounal of mathematical analysis and applications 76, 1980, p. 571-599.

LIU X.-S., [1987], "Interior operation, regularity and normality of topology on a completely distributive lattice", Kexue Tongbao [Chinese Science Bulletin] 21, 1987, p. 1606-1608 [en chinois]. 
LOWEn R., [1974] Theory of fuzzy topologies, Thèse, Bruxelles, Université Libre de Bruxelles. LOWEN R., [1976], "Fuzzy topological spaces and fuzzy compactness", J. Math. Anal. Appl. 56, 1976, p. 621-633.

MaC Lane S., [1971], Categories for the Working Mathematician, New York, Springer-Verlag, 1971, [2 éd., 1998].

maC Lane S., MoerdiJK I., [1991], Sheaves in Geometry and Logic, a first introduction to topos theory, New York, Springer-Verlag, 1992, 2e éd., 1994.

MATHERON G., SERRA J., [1988], "Strong filters and connectivity", Image analysis and Mathematical morphology 2, London, Academic Press, 1988, p. 141-157.

PU S.-L., [1982], "A better definition of boundary of a fuzzy set", Fuzzy Mathematics 2[1]; 1982, p. 23-34 [en chinois avec un récapitulatif en anglais].

RIBeIro H., [1942], « Sur les espaces à métrique faible », Portugaliae Mathematica 4[1], 1943, p. 21-39, [avec une correction p. 65-68].

RIESZ F., [1907], "Die Genesis des Raumbegriffes", Math. és phys. lapok 16, 1907, p. 145-161.

RIESZ F., [1909], "Die Genesis des Raumbegriffes", Math. Naturwiss. Ber. Ungarn 24, 1909, p. 309353; Euvres complètes 1, Budapest, Paris, Académie des sciences de Hongrie, Gauthiers-Villars, 1960, p. 110-154.

SAMPATH KUmar s., [2000], "Connectedness in fuzzy bitopological spaces", J. Fuzzy Math. 8[3], 2000, p. 651-659.

šostak (shostak) A.P., [1985], "On a fuzzy topological structure", Suppl. Rend. Circ. Mat. Palermo, 2 [11] (1985), p. 89-103.

ŠOSTAK (SHOSTAK) A.P., [1989], "Two decades of fuzzy topology : basic ideas, notions and results", Russian Math. Surveys 44[6], 1989, p. 125-186.

THRON W.J., [1997], "Frederic Riesz'contributions to the foundations of general topology", Aull C.E. et Lowen R. (eds), Handbook of the history of general topology, vol. 1, Dordrecht, Kluwer Academic Publishers, 1997.

TURANLI N., ÇOKER D., [1993], "On fuzzy supratopological spaces and fuzzy supraconnected spaces", J. Fuzzy Math. 1[4], 1993, p. 811-821.

WARren R.H., [1977], "Boundary of a fuzzy set", Indiana Univ. Math. J. 26[2], 1977, p. 191-197.

WILSON w.A, [1931], "On quasi-metric spaces", American J. Math. 53, 1931, p. 675-684.

WU G.-Q., ZHENG C.-Y., [1987], "Fuzzy boundary and characteristic properties of orderhomomorphisms", Fuzzy Sets and Systems 39, 1991, p. 329-337.

YING M., [1987], "A new approach for fuzzy topology (I)", Fuzzy Sets and Systems 39, 1991, p. 303321.

YING M., [1989], "A new approach for fuzzy topology (II)", Fuzzy Sets and Systems 47, 1992, p. 221-232.

ZADEH L.A., [1965], "Fuzzy sets", Information and Control 8, 1965, p. 338-353. 